\title{
Blocking gephyrin phosphorylation or microglia BDNF signaling prevents synapse loss and reduces infarct volume after ischemia.
}

Teresa Cramer ${ }^{1 *}$, Raminder Gill2*, Zahra S Thirouin ${ }^{1 *}$, Markus Vaas ${ }^{1}$, Suchita Sampath ${ }^{1}$, Fanny Martineau ${ }^{4}$, Sara B. Noya ${ }^{1}$, David Colameo ${ }^{1}$, Philip K.-Y. Chang ${ }^{2}$, Peiyou Wu ${ }^{2}$, Philip A Barker ${ }^{3}$, Steven A. Brown ${ }^{1}$, Rosa C. Paolicelli ${ }^{4}$, Jan Klohs ${ }^{5}$, R. Anne McKinney²\#, Shiva K. Tyagarajan ${ }^{1} \#$

1. Institute of Pharmacology and Toxicology, University of Zurich, Winterthurerstrasse 190, $\mathrm{CH}$ 8057, Switzerland

2. Department of Pharmacology and Therapeutics, McGill University, 3649 Prom. Sir-WilliamOsler, Montreal, QC, H3G OB1, Canada

3. Department of Biology, University of British Columbia, 3187 University Way, ASC 413 Kelowna, $B C$, V1V 1V7, Canada

4. Department of Biomedical Sciences, University of Lausanne, Rue du Bugnon 7 - CH-1005 Lausanne

5. Institute for Biomedical Engineering, University of Zurich and ETH Zurich, Wolfgang-PauliStrasse 27, CH 8093, Switzerland

* equal contribution

\#Co-senior: tyagarajan@pharma.uzh.ch and anne.mckinney@,mcgill.ca 


\begin{abstract}
:
Microglia interact with neurons to facilitate synapse plasticity; however, signal transducers between microglia and neuron remain unknown. Here, using in vitro organotypic hippocampal slice cultures and transient MCAO in genetically-engineered mice in vivo, we report that at $24 \mathrm{~h}$ post-ischemia microglia release BDNF to downregulate glutamatergic and GABAergic synapses within the peri-infarct area. Analysis of the CA1 hippocampal formation in vitro shows that proBDNF and mBDNF downregulate glutamatergic dendritic spines and gephyrin scaffold stability through $\mathrm{p} 75^{\mathrm{NTR}}$ and TrkB receptors respectively. Post-MCAO, we report that in the periinfarct area and in the corresponding contralateral hemisphere similar neuroplasticity occur through microglia activation and gephyrin phosphorylation at Ser268, Ser270 in vivo. Targeted deletion of the Bdnf gene in microglia or GphnS268A/S270A (phospho-null) point-mutations protect against ischemic brain damage, neuroinflamation and synapse downregulation normally seen post-MCAO. Collectively, we report that gephyrin phosphorylation and microglia derived BDNF faciliate synapse plasticity after transient ischemia.
\end{abstract}




\section{Introduction:}

Ischemic stroke is a leading cause of death and long-term disability worldwide. The annual mortality rate of 5.5 million is further compounded by high morbidity as up to $50 \%$ survivors are chronically disabled ${ }^{1}$. Therapeutic approaches to CNS ischemia developed in the laboratory have focused on mechanisms contributing to ischemic damage, namely excitotoxicity, oxidative stress and inflammation ${ }^{2,3}$. Unfortunately to date clinical trails targeting glutamate receptors ${ }^{4}$, GABA receptors ${ }^{5}$, calcium channels ${ }^{6}$, sodium channels ${ }^{7}$ and free radicals ${ }^{8}$ have all failed. The lack of treatment options is directly related to our poor understanding of the possible mechanisms underlying the disease. As the brain has developed inherent mechanism(s) for self-preservation; gaining insights into these protective measures may thus provide a way forward to counteract ischemic brain injury.

Typically, rapid cell death occurs in the ischemic core but intriguingly neurons in the periinfarct area, a region with constrained blood flow and partially preserves energy metabolism, survive. Within the peri infarct the propagating neuronal depolarization in combination with impairment of glia function causes an increased extracellular concentration of ions and neurotransmitters resulting in neuronal functional perturbations ${ }^{9}$. This is accompanied by reductions in both excitatory dendritic spines ${ }^{10}$ and GABAergic synapses ${ }^{11}$. Interestingly, the neurotrophin brain-derived neurotrophic factor (BDNF) can decrease cell death and ischemic core volume leading to improvement of neurological outcome after experimental stroke either upon overexpression in vivo using genetic methods ${ }^{12}$ or upon exogenous application ${ }^{13}$. Correspondingly, inhibition of BDNF exacerbates ischemic damage ${ }^{14}$.

Under physiological conditions, glutamatergic neurotransmission induces BDNF expression ${ }^{15}$. BDNF is expressed as a proprotein, proBDNF, and is subsequently processed to its 
mature form $\mathrm{mBDNF}{ }^{16}$. proBDNF preferentially binds to the low-affinity nerve growth factor receptor $\mathrm{p} 75^{\mathrm{NTR}}$ and negatively regulates dendritic spine stability through Rho GTPases, Rho/Rac1 activation ${ }^{17}$. mBDNF signals through the neurotrophin receptor tyrosine kinase $\mathrm{B}(\mathrm{TrkB})$ to enhance excitatory neurotransmission ${ }^{18}$. mBDNF can also bind to $\mathrm{p} 75^{\mathrm{NTR}}$ receptors, albeit with much lower affinity ${ }^{19}$. At inhibitory GABAergic synapses, mBDNF induces the internalization of $\mathrm{GABA}_{\mathrm{A}}$ receptors ${ }^{20}$ and downregulation of the main inhibitory synapse scaffolding protein gephyrin ${ }^{21}$, thereby reducing GABAergic transmission in principal cells.

Ischemia also activates inflammatory pathways that subsequently recruit leukocytes to the injured area of the brain ${ }^{22}$. Immune cells contribute to both neuroprotection and programmed cell death ${ }^{23}$, suggesting that the temporal window of inflammation might determine cell survival or death. The immune response signalling events must be counteracted to mitigate tissue damage and re-establish homeostasis. Microglia the resident immune cells in the central nervous system (CNS) are the primary responders during defense. They clear cellular debris as part of the tissue repair and wound healing processes ${ }^{24,25}$. In recent years, microglia have also been shown to play an essential role in synapse pruning during postnatal brain development ${ }^{26}$. Microglia activation can be triggered by acute insult, causing process elongation and increased expression of marker proteins like IBA1 and CD11b ${ }^{27}$. Microglial activation can protect the brain, albeit the precise cellular and molecular mechanisms for microglia influenced neuroprotection remain unclear. Microglia processes can directly sense synaptic activity ${ }^{28}$, and can regulate neuronal calcium load and functional connectivity through neuronal mitochondrial function and P2Y12 receptor activation on contacting microglia ${ }^{29}$. Subsequent inflammation induced by lipopolysaccharide (LPS) activates downstream calcium-calmodulin-dependent kinase (CaMKIV), cyclic AMP 
response element binding protein (CREB) phosphorylation and BDNF protein increase facilitate neuron survival after cortical injury ${ }^{30}$.

It has been reported that BDNF administered either intravenously ${ }^{13}$, with viral vectors ${ }^{12}$, or by addition of the bioactive high-affinity TrkB agonist, 7,8-dihydroxyflavone, can protect neurons from apoptosis and decrease infarct volumes in animal models of stroke ${ }^{31}$. These findings suggest that elevated BDNF is beneficial for recovery after stroke. However, the mechanisms underlying the beneficial effect of BDNF post-ischemia remain unclear. Here, we set out to assess the physiological mechanism(s) that are triggered upon ischemic brain damage to enable tissue repair and neural network reorganization. Using organotypic hippocampal slice cultures and the oxygen-glucose deprivation (OGD) cellular model of ischemia, we report that within the first 90 min post-ischemia, proBDNF via $\mathrm{p} 75^{\mathrm{NTR}}$ disrupts glutamatergic, and mBDNF via TrkB disrupts GABAergic neurotransmission. We found that ERK1/2 and GSK3 $\beta$ pathways downstream of TrkB phosphorylate gephyrin at Ser 268 and Ser 270 residues resulting in GABAergic synapse loss. Using transient middle cerebral artery occlusion (MCAO) in wildtype and geneticallyengineered mice, we uncover a central role for BDNF derived from microglia in influencing gephyrin phosphorylation downstream of TrkB receptor signaling. Using pharmacological depletion of microglia, CRISPR/Cas9 generated GphnS268A/S270A mutant mouse, and Bdnf gene deletion from microglia, we consistently demonstrate reduced microglial activation and enhanced synapse preservation at $24 \mathrm{~h}$ post MCAO. Collectively, these observations unravel microglia derived BDNF as the signal transducer linking microglia and neurons, to activate ERK1/2 and GSK3 $\beta$ pathways to influence glutamatergic and GABAergic synapse integrity through gephyrin phosphorylation. 


\section{Results:}

\section{OGD causes glutamatergic and GABAergic synapse downregulation}

To understand the mechanisms of BDNF action in ischemia we started with an in vitro model of ischemia, OGD, in organotypic hippocampal slice cultures obtained from transgenic mice that express myristoylated GFP in a subset of CA1 pyramidal neurons ${ }^{32}$ and studied synaptic changes in the CA1 area after OGD (4 min) and recovery at $90 \mathrm{~min}$ and $24 \mathrm{~h}$. First, we confirmed that we had induced hypoxia with OGD by measuring hypoxia-inducible factor $1 \alpha(\mathrm{HIF} 1 \alpha)$ expression ${ }^{33}$ and found 1.5-fold increase in HIF1 $\alpha$ expression in area CA1 90 min after OGD (Suppl. Fig. 1a, b). We then determined glutamatergic synapse alterations after OGD by measuring changes in dendritic spines ${ }^{34}$. We observed an overall significant reduction in spine density on CA1 pyramidal neurons at both 90 min and $24 \mathrm{~h}$ following OGD compared to control cultures (Fig. 1a, b; Supp. Fig. 1b). The mushroom and long-thin subtype of spines were particularly affected (Fig. 1b).

Next, we evaluated OGD-induced changes at GABAergic synapses in area CA1 at 90 min and $24 \mathrm{~h}$ following OGD. We immunolabeled for inhibitory presynaptic VGAT and postsynaptic inhibitory scaffolding protein gephyrin. We found a significant overall downregulation in gephyrin cluster density 90 min following OGD compared to control (Fig. 1c-e) in the Stratum Radiatum. However, after $24 \mathrm{~h}$ gephyrin cluster density remained significantly reduced, while cluster volume had recovered to baseline (Suppl. Fig. $1 b^{\prime}-b^{\prime \prime}$ ) similar to untreated cells.

To determine whether these morphological changes were accompanied by a functional deficit, we recorded AMPA-mediated miniature excitatory postsynaptic currents (mEPSCs) from CA1 pyramidal neurons within $24 \mathrm{~h}$ after OGD (Fig. 1f-f'). We found that the input resistance and resting membrane potential of CA1 hippocampal pyramidal cells in OGD and sister untreated cells 
were similar, suggesting that OGD does not impact receptor open probability or intracellular chloride concentration. However, we found a significant decrease in the mEPSC amplitude in OGD-treated slices compared to control (Fig. 1f). Similarly, the inter-event-interval (IEI) of mEPSC of OGD cells were increased compared to control (Fig. 1f'). Taken together, dendritic spine loss is mirrored by functional loss of excitatory synapses $24 \mathrm{~h}$ after OGD. Subsequently, to determine whether inhibitory circuitry was also affected we recorded $\mathrm{GABA}_{\mathrm{A}}$-mediated miniature inhibitory postsynaptic current (mIPSC) within 24 h of OGD induction. mIPSC analysis showed no observable changes in the amplitude of mIPSC after OGD compared to control slices (Fig. 1g). However, a significant increase in the IEI in OGD-treated slices was observed (Fig. 1g'). These functional data recapitulate the morphological observations that inhibitory synapse loss after OGD does not recover, but total $\mathrm{GABA}_{\mathrm{A}} \mathrm{Rs}$ at synaptic sites within the existing synapses recover at 24 $\mathrm{h}$ after OGD.

\section{Scavenging BDNF after OGD using TrkB-Fc rescues OGD-induced synapse deficit}

As BDNF is upregulated after ischemia ${ }^{35,36}$, we assessed whether BDNF signaling contributed to synapse loss on CA1 pyramidal neurons after OGD. We scavenged proBDNF and mBDNF using chimeric TrkB-Fc $(10 \mu \mathrm{g} / \mathrm{mL})$ and exposed organotypic hippocampal slices to $4 \mathrm{~min}$ OGD. Dendritic spine quantification in CA1 pyramidal neurons showed prevention of total spine density loss caused by OGD in TrkB-Fc treated cultures in comparison to untreated cultures (Fig. 2a, b). Specifically, the OGD-induced decrease in mushroom and long-thin subtype of dendritic spines was prevented by TrkB-Fc (Fig. 2a, b). We could confirm that TrkB-Fc scavenges both pro- and mBDNF by performing co-immunoprecipitation against TrkB-Fc and Western blot analysis against proBDNF and mBDNF (Fig. 2b'). Once we confirmed that TrkB-Fc also scavenges 
mBDNF, we assessed whether TrkB-Fc treatment also protects GABAergic synapses. We found that post-synaptic gephyrin clustering was protected by TrkB-Fc in OGD treated cultures (Fig. 2ce). Importantly, TrkB-Fc caused no detectable effect in control cultures (not exposed to OGD).

To determine whether morphological synapse protection is recapitulated functionally, we recorded excitatory AMPA-mediated mEPSC from CA1 pyramidal neurons from all groups. We found that the OGD-induced reduction of mEPSC amplitudes $24 \mathrm{~h}$ following OGD was prevented by TrkB-Fc treatment, being comparable to treated and untreated controls (Fig. 2f; Suppl. Fig. 1cc'). Similarly, increase in IEI was also prevented by TrkB-Fc treatment as seen $24 \mathrm{~h}$ after the induction of OGD, with values similar to treated and untreated control slices (Fig. 2f'; Suppl. Fig. $\left.1 c^{\prime}\right)$, indicating that the reduced occurrence of mEPSC after OGD was due to BDNF signaling.

To test if TrkB-Fc also prevented changes in inhibitory transmission we recorded $\mathrm{GABA}_{\mathrm{A}}-$ mediated mIPSC from CA1 pyramidal neurons from all groups. We found that mIPSC amplitudes were comparable in all groups (Fig. 2g, g'; Suppl. Fig. 1d). The previously observed increase in IEI caused by OGD was prevented with TrkB-Fc treatment to control levels (Fig. 2g'; Suppl. Fig. $\left.1 d^{\prime}\right)$, confirming that the OGD-induced decrease in glutamatergic and GABAergic synapse loss is mediated by BDNF.

proBDNF and $m B D N F$ signal via $p^{75^{N T R}}$ and TrkB receptors to induce glutamatergic and

\section{GABAergic synapse loss respectively after ischemia}

Next we investigated the molecular pathways involving BDNF-mediated synapse loss at 90 min post OGD in organotypic hippocampal slice cultures. To specifically investigate the contribution of proBDNF on OGD-induced dendritic spine loss, we used blocking antibodies to either inhibit proBDNF or $\mathrm{p} 75^{\mathrm{NTR}}$. Pre-treatment of OGD slices with either anti-proBDNF or anti-p $75^{\mathrm{NTR}}$ 
antibodies prevented OGD-induced dendritic spine loss (Fig. 3a-c; $\mathrm{P}=0.37$ ). Additionally, treatment of OGD slices with anti-proBDNF or anti-p $75^{\mathrm{NTR}}$ antibody did not prevent gephyrin cluster loss after OGD (Fig. 3d; $\mathrm{P}=0.52$ ). These findings indicate that pro-BDNF signaling through p75 ${ }^{\mathrm{NTR}}$ to specifically induces excitatory synapses loss following OGD.

We next investigated the role of mBDNF in OGD-induced synapse loss. For this we pretreated cultures with anti-mBDNF (N-9, a function blocking antibody) prior to OGD and then quantified the dendritic spines; control sister cultures were processed simultaneously with and without anti-mBDNF treatment. The data revealed a significant downregulation of total dendritic spines in both OGD and anti-mBDNF pretreated OGD slices, compared to control and cultures pretreated with anti-mBDNF (Fig. 3e-f). Similar to our earlier observations, only the mushroom and long-thin subtype of dendritic spines were downregulated 90 min following OGD with or without anti-mBDNF treatment (Fig. 3f). This data suggested to us that mBDNF was not mediating excitatory synapse loss after OGD. In contrast, anti-mBDNF treatment was sufficient to prevent the reduction of gephyrin cluster density in OGD treated slices (Fig. 3g). Reduction of gephyrin cluster volume was also prevented in the presence of anti-mBDNF antibody compared to OGD slices (Fig. 3h). Our results show that mBDNF acts specifically on GABAergic synapses after OGD.

\section{Blocking ERK1/2 and GSK3ß pathways protects gephyrin, but not dendritic spine loss}

mBDNF binds with high-affinity to TrkB receptors. It is also known that ERK1/2 and GSK3 $\beta$ pathways are activated downstream of TrkB ${ }^{37}$. Therefore, we determined whether ERK1/2 and GSK3 $\beta$ signaling cascades downstream of TrkB were activated after OGD to mediate gephyrin cluster reduction at GABAergic terminals. We pretreated slices with pharmacological inhibitors 
of GSK3 $\beta(25 \mu \mathrm{M}$ GSK3 $\beta$-IX) and MEK (30 $\mu \mathrm{M}$ PD98059) to prevent activation of these kinases before OGD. Control treated and untreated cultures served for comparative analysis. As expected, inhibiting ERK1/2 and GSK3 $\beta$ pathways did not prevent dendritic spine loss in OGD treated cultures (Fig. 4a, b). Specifically, the mushroom and stubby dendritic spines that were most affected by OGD could not be rescued with ERK1/2 and GSK3 $\beta$ blockade (Fig. 4b). However, at GABAergic synapses gephyrin cluster loss was prevented after OGD in slices pretreated with GSK3 $\beta$-IX and PD98059, compared to untreated OGD slices (Fig. 4a, c, d).

Previously, we have reported that GSK3 $\beta$ phosphorylates gephyrin on serine 270 (ser270) to negatively regulate the number of gephyrin clusters ${ }^{38}$, and ERK1/2 phosphorylates gephyrin at serine 268 (ser268) to negatively regulate the size of gephyrin clusters ${ }^{39}$. In order to determine whether phosphorylation of these serine residues were important for OGD-induced gephyrin downregulation, we used biolistic transfection of GFP-tagged gephyrin where serines 268 and 270

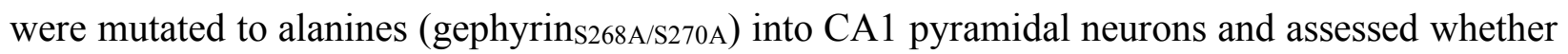
this gephyrin mutant is insensitive to mBDNF-mediated TrkB signaling after OGD. Our analysis showed that gephyrin ${ }_{\mathrm{S} 268 \mathrm{~A} / \mathrm{S} 270 \mathrm{~A}}$ mutant is resistant to OGD compared to wildtype gephyrin (gephyrinwT) (Fig. 4e, f, h, i). Transgene expression of gephyrin ${ }_{S 268 \mathrm{~A} / \mathrm{S} 270 \mathrm{~A}}$ mutant could not prevent dendritic spine loss after OGD, which is consistent with the data using pharmacological inhibitors that block kinase pathways directly phosphorylating gephyrin at Ser268 and Ser270 respectively

(Fig. 4g). Overall, our results identify gephyrin S268 and S270 phosphorylation downstream of TrkB as a determinant for GABAergic synapse loss after OGD.

\section{The MCAO model in vivo recapitulates OGD-induced synapse loss at $24 \mathrm{~h}$ post ischemia}


In order to confirm our in vitro OGD results also occurred in in vivo we used MCAO technique, in which an intraluminal filament is used to cause transient ischemia in the fronto-parietal cortex and striatum ${ }^{40}$, and assayed for glutamatergic and GABAergic synapse loss $24 \mathrm{~h}$ post MCAO. MCAO is the most extensively used model in rodents as it produces a reproducible infarct (core and peri infarct area) where pathophysilogical cascades are well described. The peri-infarct area surrounding the core is the site for inflammation, synaptic plasticity and circuit adaptations where structural and functional changes within cortex have been observed in patients after 3 months following an anterior ischemic stroke ${ }^{41}$. Cell death within the ischemic core renders the tissue fragile for morphology or functional analysis; hence, we restricted our analysis to the penumbra.

We used immunohistochemical staining to assess changes in glutamatergic and GABAergic synapse changes within the peri-infarct area and in the corresponding area contralaterally as comparison. For glutamatergic synapse labeling we chose VGLUT1 to test for glutamatergic presynaptic changes and PSD95 for glutamatergic postsynaptic changes (Fig. 5a, b, c). For GABAergic synapse labeling we chose GAD65/67 to label presynaptic terminals; $\mathrm{GABA}_{A} \mathrm{R} \gamma 2$ for synaptic receptors and $\mathrm{GABA}_{\mathrm{A}} \mathrm{R} \alpha 5$ for extrasynaptic receptors (Fig. 5a, d, e, f). Analysis of parietal cortex layer 2/3 (L2/3) $24 \mathrm{~h}$ after MCAO did not show any observable loss of PSD95 (Fig. 5c). This result is consistent with previous report that, under ischemic conditions, nNOS interacts with PSD95 to stabilize it at the cell membrane ${ }^{42}$. Analysis for VGLUT1-positive terminals showed a significant reduction in presynaptic sites in both ipsi- and contra-lateral hemispheres (Fig. 5b).

At GABAergic synapses, we saw a significant reduction in GAD65/67 terminals, $\gamma 2$ subunit-containing synaptic $\mathrm{GABA}_{\mathrm{A}} \mathrm{Rs}$ and $\alpha 5$ subunit-containing extrasynaptic GABA $\mathrm{A}_{\mathrm{R}}($ Fig. 5d-f). We could not morphologically assess for $\alpha 1$ and $\alpha 2$ subunit-containing GABA $\mathrm{A}_{\mathrm{A}}$ in MCAO 
tissue due to the strong post-fixation protocol that is not condusive for these two antibodies. Therefore, we used Western blot (WB) to examine the expression level of $\alpha 1$ and $\alpha 2$ subunits in control and MCAO tissue from both ipsi- and contra-lateral hemispheres of parietal cortex L2/3. We found a significant reduction in $\alpha 1$ and $\alpha 2 \mathrm{GABA}_{\mathrm{A}} \mathrm{R}$ subunit expression after MCAO (Suppl. Fig 2a, b). Overall, our analysis confirms that both ipsi- and contra-lateral hemispheres decrease protein expression of important glutamatergic and GABAergic synaptic markers. Therefore, reduced excitatory and inhibitory synapse markers in vivo recapitulate our in vitro OGD results showing impaired glutamatergic and GABAergic synaptic transmission.

Blocking effector kinases downstream of TrkB in vitro OGD experiments effectively rescued GABAergic synapse loss (Fig. 4c-d) and transgene expression of gephyrin S268A/S270A mutant insensitive to ERK1/2 and GSK3 $\beta$ kinases prevented gephyrin cluster loss after OGD (Fig. 4h-i). Hence, we assessed ERK1/2 and GSK3 $\beta$ kinase activation levels $24 \mathrm{~h}$ after MCAO in frontoparietal cortex ipsi- and contralaterally in BL6 WT mice. WB analysis for ERK1/2 and its phosphorylated form showed unchanged ERK1/2 levels but significantly increased levels of phosphorylated ERK1/2 in the ipsi- but not the contra-lateral hemisphere at $24 \mathrm{~h}$ after MCAO (Fig. $5 \mathrm{~g}, \mathrm{~h})$. WB analysis for GSK3 $\beta$ showed increased kinase expression in the contra-lateral hemisphere but not the ipsi-lateral hemisphere at $24 \mathrm{~h}$ after MCAO (Fig. 5i). Gephyrin expression level and its phosphorylation at Ser 268 and Ser 270 sites changed at 24 h after MCAO (Fig. 5jj','). WB quantification confirmed that total gephyrin protein levels significantly decrease at $24 \mathrm{~h}$ post MCAO (Fig. 5j'). Consistent with our observation of elevated ERK1/2 activation on the ipsilateral hemisphere, we observed significantly higher S268 phosphorylation on gephyrin (Fig. 5j',). Similarly, higher GSK3 $\beta$ levels in the contra-lateral hemisphere correlated with significantly higher gephyrin S270 phosphorylation (Fig. 5j'"'). These observations are consistent with our in 
vitro OGD data, and further confirm a role for ERK1/2 and GSK3 $\beta$ pathways in directly phosphorylating gephyrin to regulate protein stability after MCAO.

\section{Synapse loss after MCAO is attenuated in GphnS268A/S270A point mutant mice}

To obtain a more direct confirmation for the central role of gephyrin phosphorylation in synapse alterations 24 h post-MCAO, we generated a GphnS268A/S270A global point mutant mouse line using CRISPR/Cas9 (Cyagen, USA). We performed MCAO in GphnS268A/S270A mutant mice and compared synapse plasticity changes in the parietal cortex L2/3 with sham GphnS268A/S270A littermates. We analysed for changes in glutamatergic and GABAergic synaptic markers using immunohistochemical analysis $24 \mathrm{~h}$ post-MCAO (Fig. 6). In the GphnS268A/S270A mutant mice, we observed stabilization of excitatory VGLUT1-positive terminals on both ipsi- and contralateral hemispheres $24 \mathrm{~h}$ post MCAO (Fig. 6a, b, b'). PSD95 cluster density was also unchanged in the ipsi- and the contra-lateral hemispheres (Fig. 6b'). Analysis of GABAergic synaptic markers showed a significant increase in the GAD65/67 puncta density in both ipsi- and contra-lateral hemispheres (Fig. 6c). Significantly, we did not observe any reduction in $\gamma 2$ - and $\alpha 5$-containing $\mathrm{GABA}_{\mathrm{A}} \mathrm{Rs}$ in $G p h n 268 / \mathrm{S} 270 \mathrm{~A}$ mutant mice $24 \mathrm{~h}$ post MCAO (Fig. 6 c'-c'’). Consistent with observed synaptic marker changes, WB analysis for the $\alpha 1$ and $\alpha 2$ subunits in Gphn268/S270A mutant mice showed no alterations for these two major $\mathrm{GABA}_{\mathrm{A}} \mathrm{R}$ subunits $24 \mathrm{~h}$ following MCAO (Suppl. Fig. 2c-d). Taken together, these results substantiate an involvement of ERK1/2 and GSK3 $\beta$ as downstream effectors that critically influence gephyrin scaffold stability along with glutamatergic and GABAergic synapse integrity post-MCAO.

\section{Microglia induce synapse loss after MCAO}


While it is well established that BDNF levels increase after cerebral ischemia, the source of BDNF after stroke remains unclear. Given that Bdnf transcripts have been localized within microglia ${ }^{43}$, we wondered whether microglia contributed to BDNF signaling after stroke. We assessed for BDNF protein changes within ionized calcium-binding adaptor protein-1 (IBA-1) positive cells corresponding to microglia using near super resolution Airy scan microscopy analysis of sham and MCAO BL6 WT mice. Under baseline sham condition, we found low BDNF colocalization within IBA1 positive microglia (Suppl. Fig. 3a, a'). However, following MCAO we could detect elevated BDNF protein within both soma and processes on both ipsi- and contra-lateral hemispheres (Suppl. Fig. 3a). Quantification confirmed an increase in BDNF protein within microglia at $24 \mathrm{~h}$ post MCAO (Suppl. Fig. 3a'), implicating elevated BDNF protein translation within microglia in the synaptic pathology of stroke.

To directly examine the role of microglia in BDNF synthesis and synaptic changes after MCAO, we next sought to deplete microglia from the brain using the pharmacological inhibitor PLX5622 that targets colony stimulating factor 1 receptor (CSF-1R) phosphorylation in microglia (Plexxikon Inc. Berkeley, CA 94710). It has been reported that prolonged administration of this drug ( 1 week) in a formulated chow diet depletes $90 \%$ of the microglia from rodent brain ${ }^{44,45}$. Replacing the mice on regular diet repopulates microglia cells within 5-7 days. No adverse changes to synapse structure and function, or transcriptional changes have been reported after PLX5622 chow administration ${ }^{46}$. We confirmed the loss of microglia after PLX5622 chow administration after 7 days (Suppl. Fig. 3b, b'). ${ }^{43}$

In order to test whether microglia contribute to synaptic changes observed at $24 \mathrm{~h}$ post MCAO, we treated BL6 WT with PLX5622 formulated chow or control chow. We then assessed 
for synapse alterations by staining for synaptic markers and found no significant differences in either glutamatergic synaptic markers (VGLUT1, PSD-95) or GABAergic synaptic markers (GA65/67, $\alpha 5$ and $\gamma 2$ GABA $_{A}$ Rs) between the groups (Suppl. Fig. 3c-h). Similarly, WB analysis to assess $\alpha 1$ or $\alpha 2 \mathrm{GABA}_{\mathrm{A}} \mathrm{R}$ subunit expression showed no changes at $24 \mathrm{~h}$ post-MCAO in mice administered with PLX5622 (Suppl. Fig. 2e, f). In addition, WB analysis to assess total gephyrin or changes in gephyrin phosphorylation at S268 and S270 in PLX5622 treated mice showed no changes (Suppl. Fig. 3i-i','). These findings point to the critical role of microglia in mediating synapse loss after MCAO.

\section{Microglia release BDNF after MCAO to induce synapse loss}

BDNF has been shown to play a critical role in the activation of microglia in vitro, and increase in BDNF has been tightly linked to pro-inflammation responses ${ }^{47,48}$. To specifically evaluate the role of BDNF within microglia in synapse loss following MCAO, we used CX3CR1 $1^{\text {ERT2Cre+/- mice }}$ specifically expressing tamoxifen-inducible Cre recombinase in microglia cells and generated BDNF flox/flox / CX3CR1 $1^{\text {CreERT2+/- }} \mathrm{cKO}$ mouse line, thereby preventing $B d n f$ expression selectively in microglia. We used BDNF ${ }^{\mathrm{wt} / \mathrm{wt}} / \mathrm{CX} 3 \mathrm{CR} 1^{\mathrm{CreERT2+/-}}$ and BDNF flox/flox / CX3CR1 $1^{\mathrm{CreERT} 2+/-}$ mice to culture microglia from post-natal day 3 pups and treated with tamoxifen to confirm $B d n f$ gene deletion in microglia cells. qRT-PCR analysis confirmed significant reduction in microglial $B d n f$

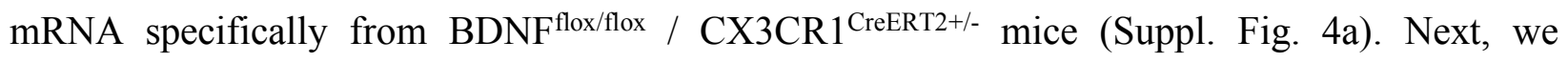

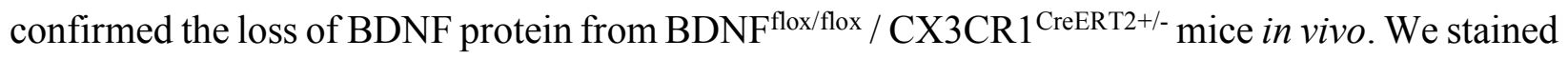
for BDNF and IBA1 in brain slices from BDNF ${ }^{\mathrm{wt} / \mathrm{wt}} / \mathrm{CX} 3 \mathrm{CR} 1^{\text {CreERT2+/- }}$ and BDNF flox/flox /

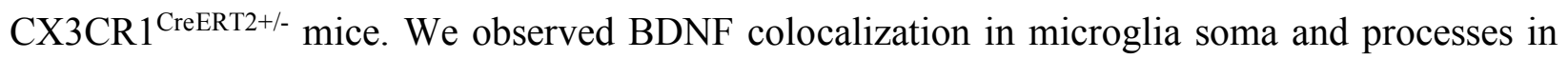
the BDNF ${ }^{\mathrm{wt} / \mathrm{wt}} / \mathrm{CX} 3 \mathrm{CR} 1^{\mathrm{CreERT2}+/-}$ tissue but not in BDNF flox/flox $/ \mathrm{CX} 3 \mathrm{CR} 1^{\mathrm{CreERT2+/}}$ mice tissue (Suppl. Fig. 4b, b’). 
Having confirmed that our cKO mouse line efficiently deletes BDNF in microglia cells, we examined the contribution of microglia Bdnf to synaptic changes observed at $24 \mathrm{~h}$ post MCAO. For this we assessed changes in synaptic markers (VGLUT1,GAD65/67, GABA $\mathrm{R} \gamma 2$ and $\left.\mathrm{GABA}_{\mathrm{A}} \mathrm{R} \alpha 5\right)$ in both ipsi- and contra-lateral hemispheres at $24 \mathrm{~h}$ post-MCAO compared to BDNF flox/flox $/$ CX3CR1 $1^{\text {CreERT2+/- }}$ sham animals. There was no significant changes between the MCAO and sham groups (Suppl. Fig. 4c-h). We also performed WB analysis to measure $\alpha 1$ and $\alpha 2 \mathrm{GABA}_{\mathrm{A}} \mathrm{R}$ subunit expression level changes between MCAO and sham group (Suppl. Fig 2g, h). Our analysis confirmed that synaptic receptor expression is unchanged upon $B d n f$ gene depletion from microglia cells post-MCAO. Our earlier observation showed stabilized gephyrin protein levels and no increase in gephyrin phopshorylation at Ser268 and Ser270 after microglia depletion using PLX5622 (Suppl. Fig. 3i- I'”,). In order to confirm if microglial BDNF signaling led to gephyrin protein loss and elevated phosphorylation at Ser268 and Ser270 residues, we performed WB analysis using tissue from BDNF flox/flox / CX3CR1 $1^{\text {CreERT2+/- }}$ sham and MCAO animals (Suppl. Fig. 4i- i','). The WB analysis showed that total gephyrin levels and gephyrin phosphorylation at S270 were unchanged, while gephyrin phosporlyation at S268 is reduced post MCAO. Our results uncover a consistent pattern of gephyrin stabilization, reduced gephyrin phosphorylation at Ser268 and Ser270 residues and synapse preservation at $24 \mathrm{~h}$ post-MCAO in BL6 mice treated with PLX5622 and BDNF flox/flox / CX3CR1 $1^{\text {CreERT2+/- }}$ transgene mice, suggesting that microglia derived BDNF signals for gephyrin phosphorylation and subsequent synapse downregulation at $24 \mathrm{~h}$ post MCAO.

Microglia have been implicated in the rapid engulfment and clearance of synapses following inflammatory brain pathology ${ }^{49}$.To assess if $B d n f$ gene deletion influenced microglia ramification post MCAO, we stained for IBA-1 in BDNF ${ }^{\mathrm{wt} / \mathrm{wt}} / \mathrm{CX} 3 \mathrm{CR} 1^{\text {CreERT2+/- }}$ and BDNF flox/flox 


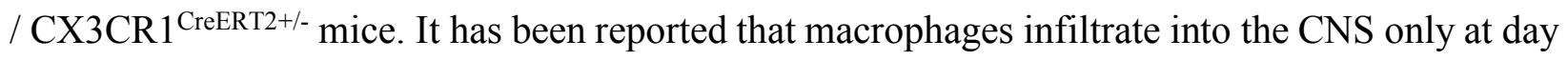
4 following $\mathrm{MCAO}^{50}$; therefore, IBA-1 positive cells are likely to be resident microglial cells. We performed 3D volume analysis of reconstructed microglia cells from ipsi- and contra-lateral hemispheres (Suppl. Fig. 5a). Quantification showed a significant volume increase in the ipsi- but not the contra-lateral hemisphere in $\mathrm{BDNF}{ }^{\mathrm{wt} / \mathrm{wt}} / \mathrm{CX} 3 \mathrm{CR} 1^{\mathrm{CreERT}+/-}$ mice, while there was no change

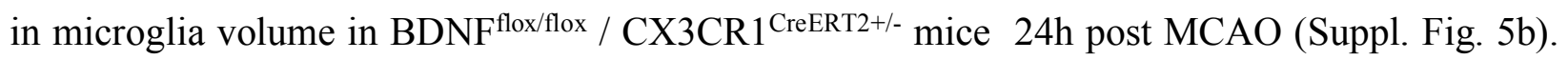
Our data shows significant hypertrophy, indicative of microglial activation, in the ipsi-lateral hemisphere of only BDNF ${ }^{\mathrm{wt} / \mathrm{wt}} / \mathrm{CX} 3 \mathrm{CR} 1^{\mathrm{CreERT2+/}}$ mice . The lack of microglial activation in

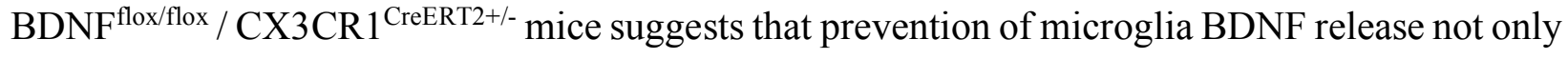
preserves synapses, but also prevents alterations in microglia morphology.

GphnS268A/S270A mutation or Bdnf deletion from microglia reduce brain damage after $M C A O$

While BDNF has been shown to play the role of pro-survival factor, including microglia activation in vivo ${ }^{14}$, there is evidence to suggest that neuronal activity-dependent exocytosis and/or release from microglia can contibute to sepcific conditions of brain pathology ${ }^{51}$. To test this, we performed MCAO in BL6 WT, BL6 WT mice treated with PLX5622, GphnS268A/S270A mutant

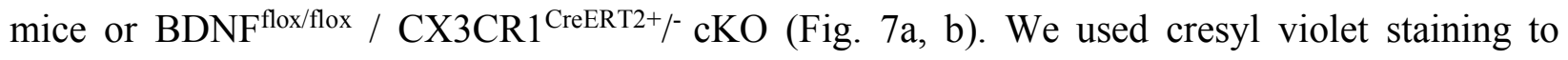
measure the infarct volume across brain sections $24 \mathrm{~h}$ following MCAO. Quantification confirmed that in GphnS268A/S270A mutant mice and BDNFflox/flox / CX3CR1 ${ }^{\text {CreERT2+/- }}$ cKO mice the ischemic tissue damage was significantly reduced as compared to BL6 WT mice. We see a trend in reduced infarct volume in PLX5622 treated mice that does not reach significance. It is likely that ablating microglia completely causes compensatory adaptations in the brain that are not 
identitical to microglia-specific $B d n f$ gene deletion. These results support our idea that prevention of microglia BDNF release after ischemia or blocking its downstream phosphorylation target gephyrin enhances tissue preservation $24 \mathrm{~h}$ post-MCAO.

In vivo inflammatory processes play a key role in tissue damage and repair. In response to inflammation, microglia acquire properties for reactive species generation and inflammatory cytokine production, and are therefore thought to be principal drivers of pro-inflammatory responses ${ }^{52,53}$. We have demonstrated above, that depletion of microglia-derived BDNF release reduces MCAO-induced increase in microglia volume (Suppl. Fig. 5). Given that GphnS268A/S270A mutant mice show enhanced tissue preservation 24h post-MCAO, we anticipated gephyrin scaffold stabilization to reduce microglia activation after MCAO. To assess microglia properties, we first examined the density of resident microglia at baseline and $24 \mathrm{~h}$ post MCAO in the brains of BL6 WT and GphnS268A/S270A mutant mice (Fig. 7c, d). Quantification of IBA-1 positive microglia showed a significant reduction of microglia density from both ipsiand contra-lateral hemispheres after MCAO in BL6 WT mice (Fig. 7c'). Similarly, analysing for area covered by the microglia cells showed significant reduction in both ipsi- and contra-lateral sides (Fig. 7c'). These observed changes are consistent with the report showing microglia migration changes after stroke ${ }^{53}$. To confirm inflamation and microglia activation status at $24 \mathrm{~h}$ post-MCAO we stained for the activation-state marker CD11b in microglial cells. Quantification for CD11b intensity showed elevated levels only on the ipsi- but not the contralateral side in BL6 WT mice (Fig. 7c'"'). Analysis of microglia density in GphnS268A/S270A mutant mice showed no changes between sham and MCAO samples (Fig. 7d'). Similarly, there was no change in the area covered (Fig. 7d"). If indeed gephyrin scaffold stabilization leads to reduced microglia activation, we anticipated less activation of microglia in GphnS268A/S270A mutant mice after 
MCAO. Analysis for CD11b intensity was not elevated after MCAO in the GphnS268A/S270A mutant mice (Fig. 7d"',). Taken together, our data shows that in Gphn268/S270A mutant mice, the mutation selectively blocks MCAO-induced microglia activation and reduces ischemic tissue damage.

\section{Bdnf deletion from microglia or GphnS268A/S270A mutation prevent BDNF increase}

If gephyrin scaffold stability contributes towards microglia activation after MCAO, then it should be reflected in proBDNF and mBDNF level changes in our different mouse lines. As a first step, we assessed for differences in the baseline level of proBDNF and mBDNF across our different mouse lines. WB analysis showed that BL6 WT mice treated with PLX5622 had elevated levels of proBDNF and BDNF ${ }^{\text {flox/flox }} / \mathrm{CX} 3 \mathrm{CR} 1^{\text {CreERT2+/- }}$ mice exhibited significantly lower levels of proBDNF, while others had levels similar to BL6 WT control (Suppl. Fig. 6a, a'). The analysis of mBDNF across different mice lines showed no significant differences (Suppl. Fig. 6a, a'”).

Once we established the baseline differences in proBDNF and mBDNF expression across mice lines used in our study, we went on to compare intra-mouse changes in proBDNF and mBDNF within ipsi- and contra-lateral hemispheres $24 \mathrm{~h}$ post-MCAO. In BL6 WT mice, we observed a significant increase in both pro- and mature- forms of BDNF specifically in the ipsilateral side; there was a small increase in the contra-lateral side but it was not statistically significant (Suppl. Fig. 6b-b'), In BL6 WT mice treated with PLX5622, we saw a significant reduction of pro-BDNF levels in both hemispheres $24 \mathrm{~h}$ post MCAO compared to sham group (Suppl. Fig. 6c-c'). This reduction suggests that resident microglia themselves are an important source of proBDNF or alternatively that microglia signal for proBDNF release elsewhere following MCAO. Levels of mBDNF showed no changes within both ipsi- and contra-lateral 
hemispheres in PLX5622 treated mice (Suppl. Fig. 6c'”). In BDNF flox/flox / CX3CR1 ${ }^{\text {CreERT2+/- }}$ cKO, although proBDNF levels were significantly lower than BL6 WT animals at baseline, there was no significant difference within the genotype after MCAO (Suppl. Fig. 6d, d'). However, mBDNF that was at similar levels to BL6 WT, showed a significant reduction in both ipsi- and contralateral hemispheres after MCAO within the genotype (Suppl. Fig. 6d-d'). We then assessed BDNF level changes in the GphnS268A/S270A mutant mice. Quantification of the WB showed that gephyrin scaffold stabilization prevented the ipsilateral increase in proBDNF and mBDNF levels observed in BL6 WT animals after MCAO (Suppl. Fig. 6e-e’'). These observations suggests that 1) microglia are an important source of BDNF after MCAO and that 2) microglial proBDNF and mBDNF release is influenced by the phosphorlyation status of gephyrin at S268 and S270 following MCAO.

We looked for direct evidence linking gephyrin phosphorylation at Ser268 and Ser270 contributing to BDNF changes within microglia after MCAO. In this regard, we stained for BDNF and IBA-1 and used near super resolutin Airy scan microscopy to quantify BDNF protein changes within microglia of $\mathrm{BDNF}^{\mathrm{wt} / \mathrm{wt}} / \mathrm{CX} 3 \mathrm{CR} 1^{\mathrm{CreERT2+/-}}, \mathrm{BDNF}^{\text {flox/flox }} / \mathrm{CX} 3 \mathrm{CR} 1^{\text {CreERT2+/- }}$ and GphnS268A/S270A mutant mice (Fig. 8a-c'). Quantification of BDNF intensity within IBA-1 cells confirmed increase within ipsi- and contra-lateral hemispheres of BDNFwt/wt /

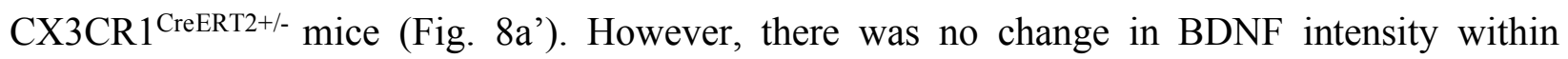

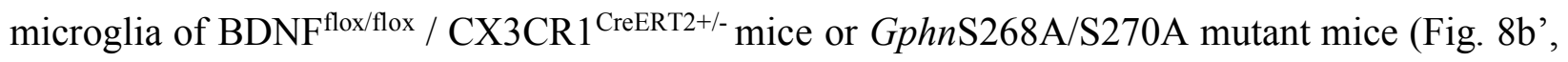
c'). These results indeed confirm that gephyrin phosphorylation regulates BDNF levels within microglia to initiate release and downstream signaling that ultimately lead to synapse loss following cerebral ischemia (see model Fig. 8d). 


\section{Discussion:}

This study reveals a direct connection between microglia, BDNF signaling and gephyrin phosphorylation as a key pathway regulating tissue integrity and synapse loss after ischemic stroke. Preventing BDNF release from microglia or preventing gephyrin phosphorylation at Ser 268 and Ser 270 are equally protective against synapse loss within 24 h post ischemic injury. Specifically, we demonstrate that (a) microglia released proBDNF and mBDNF act through p75 ${ }^{\mathrm{NTR}}$ and TrkB receptors respectively to facilitate glutamatergic and GABAergic synapse loss after ischemia; (b) ERK1/2 and GSK3 $\beta$ kinase phosphorylate gephyrin at Ser 268 and Ser 270 downstream of TrkB for GABAergic synapse downregulation; (c) in vivo microglia-specific Bdnf gene deletion or expression of gephyrin phospho-null S268A/S270A mutant protect against synapse loss at $24 \mathrm{~h}$ post-MCAO; (d) GphnS268A/S270A mutant mice prevent microglia activation and synapse loss at $24 \mathrm{~h}$ post-MCAO; (e) the mouse lines tested in our study consistently indicate similar changes within both ipsi- and contra-lateral hemispheres which is rarely addressed in stroke studies. Taken together, microglia activation and BDNF secretion are tightly coupled to glutamatergic and GABAergic synapse integrity in our model of ischemia-induced brain injury.

\section{GABAergic system in ischemia}

Our results concur with previous observations in the gerbil where following transient cerebral ischemia (24 h) $\mathrm{GABA}_{\mathrm{A}} \mathrm{Rs}$ are downregulated ${ }^{11}$. In mice, it was reported that tonic GABA currents are increased in the peri-infarct area 3 days after ischemia. An impairment in GABA transporter GAT3/GAT4 function was shown to contribute towards upregulation of extrasynaptic $\alpha 5$ and $\delta \mathrm{GABA}_{\mathrm{A}} \mathrm{R}$ tonic current ${ }^{54}$. In this study, we report a reduction in synaptic $\mathrm{GABA}_{\mathrm{A}} \mathrm{R}$ subunits $\alpha 1, \alpha 2, \gamma 2$ and also extrasynaptic $\alpha 5$ subunit expression at $24 \mathrm{~h}$ after ischemia (Fig. 5; 
Suppl. Fig. 2). In addition, we report that presynaptic GAD65/67 terminals are also significantly reduced at $24 \mathrm{~h}$ after ischemia. Importantly, our data indicates that by stabilizing GABAergic synaptic and extrasynaptic transmission, one can reduce ischemic brain damage (Fig. 7a). It has also been reported that inverse agonists specific for $\alpha 5$-subunit-containing extrasynaptic $\mathrm{GABA}_{\mathrm{A}}$ receptors administered 4 days after stroke promotes early stroke recover ${ }^{54}$. Hence, it is conceivable that increase in extrasynaptic $\mathrm{GABA}_{\mathrm{A}}$ Rs observed at day 4 post ischemia is a homeostatic response to the early reduction in GABA and synaptic $\mathrm{GABA}_{\mathrm{A}}$ Rs. Stabilizing the gephyrin scaffold could thus explain the neuroprotection observed in Gphn268A/S270A mutant mice at $24 \mathrm{~h}$ post ischemia.

\section{Postsynaptic scaffold stability after ischemia}

In WT mice, $24 \mathrm{~h}$ post MCAO gephyrin protein levels are reduced (Suppl. Fig. 3e); however, we observe no change in PSD95 clusters, as a proxy for glutamatergic post-synaptic sites (Fig. 5c). Previous studies have revealed that after ischemia nNOS interaction with PSD95 stabilizes the protein at postsynaptic sites ${ }^{42}$. Interestingly, gephyrin has also been reported to interact with nNOS

55. However, it remains to be tested whether interaction with nNOS is influenced by ERK1/2 and GSK3 $\beta$ phosphorylation of gephyrin. It is possible that reduced gephyrin expression after MCAO might make more nNOS available for PSD95 interaction and stabilization. If neuroinflammation is the trigger for nNOS activation, our data shows reduced inflammation in GphnS268A/S270A mutant mice and BDNF flox/flox; $\mathrm{CX} 3 \mathrm{CR} 1^{\text {CreERT2+/- }} \mathrm{cKO}$ mice. Therefore, BDNF signaling could increase neuroinflammation by activating nNOS after ischemia.

proBDNF and mBDNF signaling regulate synapse downregulation 
At the neuromuscular junction, proBDNF and mBDNF elicit opposite effects by promoting axon retraction through activation of $\mathrm{p} 75^{\mathrm{NTR}}$ on presynaptic site or potentiate synapse through $\operatorname{TrkB}$ activation at the postsynaptic site respectively. High-frequency neuronal activity controls the ratio of extracellular proBDNF/mBDNF by regulating the secretion of extracellular proteases, serving as a reward signal to stabilize synaptic contacts and strengthen neurotransmission ${ }^{56}$ However, within hippocampal neurons, proBDNF has been reported to activate $\mathrm{p} 75^{\mathrm{NTR}}$ localized in dendritic spines of CA1 neurons and enhance NR2B-dependent LTD ${ }^{57}$.

In the current study, we present evidence for proBDNF and mBDNF in glutamatergic and GABAergic synapse down regulation after ischemia. $\mathrm{p} 75^{\mathrm{NTR}}$ lack intrinsic enzymatic activity and activate signal transduction pathway by associating with adaptor proteins that are distinct from TrkB signaling cascade ${ }^{58}$. Consistent with this literature, our data shows the specificity for proBDNF and $\mathrm{p} 75^{\mathrm{NTR}}$ signaling for spine downregulation and mBDNF and TrkB for gephyrin regulation at GABAergic postsynaptic sites (Fig. 3). Our study has important implications. First, we uncover a synaptic plasticity function for proBDNF and $\mathrm{p} 75^{\mathrm{NTR}}$ in the ischemic brain, which is in marked contrast to its role in regulating neuronal apoptosis. Second, our results show that TrkB and downstream pathways (ERK1/2 and GSK3 $\beta$ ) specifically influence the stability of shaft synapse and of not spine synapses (Fig.4).

\section{Microglia as a source of BDNF in ischemia}

Deletion of BDNF from specific subpopulations of neurons has revealed that both presynaptic and postsynaptic BDNF contributes to specific aspects of LTP. For example, presynaptic BDNF was documented to increase the strength of LTP, while postsynaptic BDNF facilitates LTP maintenance ${ }^{59}$. In addition, BDNF release from dendritic spines can activate NMDA and TrkB 
receptors within the same release site to influence structural plasticity ${ }^{60}$. At GABAergic terminals, time duration of exogenous BDNF exposure has opposite effects on GABA $\mathrm{R}$ and gephyrin clustering. In hippocampal neuron culture, short-term (5 min) BDNF application inhibits $\mathrm{GABA}_{\mathrm{A}} \mathrm{R}$ internalization through phosphoinositide-3 kinase (PI-3 kinase) and PKC pathways (Jovanovic et al., 2004). However, long-term (90 min) BDNF application reduces GABA $\mathrm{R}$ and gephyrin clustering ${ }^{20}$. Presynaptically, BDNF regulates GAD65 mRNA expression through the recruitment of ERK pathway, leading to cAMP-response element (CRE)-binding protein (CREB) activation $^{61}$.

To add to this complexity, BDNF is not only found in neurons but also expressed in both astrocytes and microglia 43,62 . Within the spinal cord circuit, BDNF activates TrkB in lamina I neurons to downregulate KCC2 (Chloride potassium symporter), thereby increasing intracellular chloride concentration and reversing GABAergic inhibition to cause neuronal depolarization ${ }^{48}$. The resulting hyperexcitability of neurons contributes to mechanical hypersensitivity. Microgliaspecific Bdnf knockout reduces PNI-induced pain ${ }^{63}$ In the current study, we report microglia as the major source of BDNF after ischemia for glutamatergic and GABAergic synapse regulation. We observed elevated proBDNF in PLX5622 treated mice in sham condition, which suggests that microglia ablation at baseline could trigger proBDNF synthesis from other cell-types as compensatory mechanism (Suppl. Fig. 6 a'). Interestingly, proBDNF levels reduce significantly in PLX5622 treated animals after MCAO, indicating that microglia play a pivotal role in de novo BDNF synthesis, we also do not know how different metalloproteases are affected after microglia depletion. The release of BDNF from microglia has been linked to purinergic receptor P2X4R activation, causing disinhibition of pain-transmitting spinal lamina I neurons ${ }^{64,65}$. The expression of P2X4R increases after exposure to proinflammatory cytokines such as INF- $\gamma^{66}$. Microglia can 
sense extracellular ATP through P2Y12R to migrate ${ }^{67}$, or P2X7R to be activated ${ }^{68}$. Hence, it is possible that within $24 \mathrm{~h}$ post ischemia inflammation activates P2X4R expression to promote BDNF protein synthesis and release.

\section{Gephyrin phosphorylation influences microglia activation}

During acute ischemic stroke natural killer (NK) cells infiltrate periinfarct areas of the brain to promote inflammation (e.g. microglia activation) and neuronal damage. Interestingly, depletion of NK cells within the first $12 \mathrm{~h}$ after MCAO attenuates neurological deficits and infarct volume ${ }^{69}$. Here, we report that microglia depletion using PLX5622 (Suppl. Fig 5) and Bdnf gene deletion specifically from microglia (Suppl. Fig. 4) prevent glutamatergic and GABAergic synapse loss and reduce the extent of infarct volume (Fig. 7a) after ischemia. Hence, it is possible that NK cells influence infarct development through microglia activation and local BDNF release to trigger glutamatergic and GABAergic synapse loss. Furthermore, in addition to activation of microglia, we uncover activation of ERK1/2 and GSK3 $\beta$ pathways (Fig. 5g-i) $24 \mathrm{~h}$ post ischemia. As a direct target of ERK1/2 ${ }^{39}$ and GSK3 ${ }^{38}$, gephyrin phosphorylation at Ser268 and Ser270 sites are significantly increased after ischemia, while total gephyrin level is decreased (Fig. 5 j-j','). Stabilization of gephyrin clusters through the expression of S268A/S270A mutant in hippocampal slice culture selectively prevents GABAergic synapse loss after ischemia (Fig. 4h, i). In GphnS268A/S270A mutant mice both glutamatergic and GABAergic synapse loss can be prevented (Fig. 6). This suggests to us that mechanisms activated after ischemia in vivo might be similar to mechanisms operational in organotypic slice cultures after OGD.

We reveal a link between gephyrin phosphorylation at Ser268 and Ser270, microglia activation and BDNF secretion (see model; Fig. 8d). Within this model, a currently unknown signal 
originating at GABAergic postsynaptic sites would activate microglia to synthesize and release proBDNF and mBDNF for downstream signaling after MCAO. This is based on our data showing BDNF protein levels do not change after MCAO in Gphn268A/S270A mutant mice, which suggests that gephyrin scaffold stability controls microglia activation. The glutamatergic spine synapse collapse through $\mathrm{p} 75^{\mathrm{NTR}}$ signaling and GABAergic synapse downregulation via gephyrin S268 and S270 phosphorylation could facilitate microglia aided stripping of VGLUT and VGAT terminals. It has been reported that microglia physically displace GABAergic presynaptic terminals after lipopolysaccharide induced inflammation ${ }^{70}$. Together, our data identifies the mechanistic basis for silencing neurotransmission at the initial $24 \mathrm{~h}$ post-MCAO.

\section{Experimental Procedures:}

\section{Ethics Statement}

All animal handling procedures were carried out consistent with guidelines set by the Canadian Council on Animal Care, the European Community Council Directives of November 24, 1986 (86/609/EEC) and approved by the cantonal veterinary office of Zurich (ZH219/15). All procedures were approved by the Animal Resource Committee of the School of Medicine at McGill University and are outlined in McGill University Animal Handling Protocol \#5057.

\section{Hippocampal Slice Cultures and Oxygen-Glucose Deprivation (OGD)}

We have chosen to study the hippocampus as it possesses a unique unidirectional network that is preserved within organotypic cultures ${ }^{71}$, making it an ideal candidate to study microcircuitry remodeling. Organotypic hippocampal slices were prepared using the roller-tube method, as 
previously described ${ }^{71}$ with transgenic mice expressing membrane-targeted MARCKS-enhanced GFP under the Thy-1 promoter in a subpopulation of CA1 cells ${ }^{72}$. We used an established model of OGD as a model of ischemia ${ }^{73}$, briefly, mature organotypic hippocampal slices were placed in a glass dish containing glucose-free Tyrode's solution (in $\mathrm{mM}: \mathrm{NaCl}, 137 ; \mathrm{KCl}, 2.7 ; \mathrm{CaCl}_{2}, 2.5$; $\mathrm{MgCl}_{2}, 2 ; \mathrm{NaHCO}_{3}, 11.6 ; \mathrm{NaH}_{2} \mathrm{PO}_{4}, 0.4 ; \mathrm{pH}$ 7.4) containing: $2 \mathrm{mM}$ 2-Deoxyglucose, $3 \mathrm{mM}$ sodium azide and $8 \mathrm{mM}$ sucrose, for 4-5 min, and were then returned to culture media for $90 \mathrm{~min}$, $24 \mathrm{~h}$ or 1 week. Control slices were exposed to Tyrode's solution (in $\mathrm{mM}$ : $\mathrm{NaCl}, 137$; $\mathrm{KCl}, 2.7$; $\mathrm{CaCl}_{2}, 2.5 ; \mathrm{MgCl}_{2}, 2 ; \mathrm{NaHCO}_{3}, 11.6 ; \mathrm{NaH}_{2} \mathrm{PO}_{4}, 0.4$; glucose, 5.6; $\mathrm{pH}$ 7.4) for 4-5 min and returned to culture media.

\section{Mouse lines}

All procedures fulfilled the ARRIVE guidelines on experimental design, animal allocation to different experimental groups, blinding of samples to data analysis and reporting animal experiments. Littermates from heterozygous breedings were used within similar genotypes and inbred animal backgrounds. We compared results within same genotypes. The GphnS268A/S270A mutant mouse was generated using CRISPR/cas9 (Cyagen, USA) in BL6 background. B6.129P2(C)-Cx3cr1tm2.1(cre/ERT2) (Stock 020940) ${ }^{74}$ mice and Bdnftm3Jae or BDNF ${ }^{\mathrm{Tg}}$ (Stock 004339) ${ }^{75}$ were obtained from Jackson Laboratory to generate BDNFwt/wt/ CX3CR1 ${ }^{\text {ERT2Cre+/- and }}$

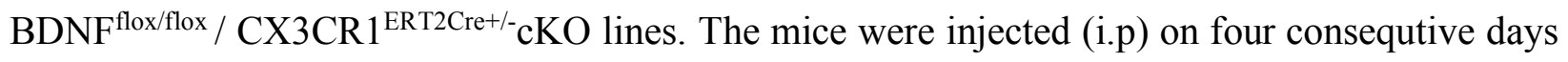
with tamoxifen dissolved in corn oil (Sigma H-6278; 1mg/ day) to induce Cre expression at 4 weeks followed by sham or MCAO surgery at 8-9 weeks of age. Animals were random assigned and both genders were used for both conditions. The PLX5622 treatment for microglia depletion followed recommended company dose (1200mg of active form of PLX5622/kg of chow). 


\section{Middle cerebral artery occlusion (MCOA) model}

Wild type C57B16/J-Crl1 mice were purchased from Charles River Laboratories (Germany), the GphnS268A/S270A mutant, BDNF ${ }^{\mathrm{Tg} / \mathrm{Tg}} / \mathrm{CX} 3 \mathrm{CR} 1^{\text {ERT2Cre+/- }}$ were bred in house. At 4 weeks age animals were randomly allocated to groups. The transient occlusion of the middle cerebral artery was conducted at 8-9 weeks age using the filament model as described previously ${ }^{76}$. Briefly, anaesthesia was induced using 3\% isoflurane in an oxygen/air (1:4) mixture and maintained at $2 \%$ Isoflurane. The area around the neck was shaved, disinfected and an incision was made along the midline. The common and the external carotid artery were isolated and ligated. A silicon rubber filament (Doccol, USA, Lot 701956RE) was inserted into the internal carotid artery to block the middle cerebral artery. The filament remained in place for $30 \mathrm{~min}$ before reperfusion was allowed by withdrawing it. During occlusion the mouse was placed in a preheated $\left(30^{\circ} \mathrm{C}\right)$ recovery box and allowed to recover from anaesthesia. After reperfusion, the internal carotid artery was ligated to prevent bleeding and the wound was sutured. Sham operation involved identical surgical procedures, but the filament was immediately withdrawn after insertion. A total volume of $500 \mu 1$ of Buprenorphine $(0.03 \mathrm{mg} / \mathrm{g})$ with Saline was injected after surgery and consecutive after $4 \mathrm{~h}$ and $8 \mathrm{~h}$ s.c. The mouse was kept for $2 \mathrm{~h}$ in the recovery box and then placed back into its home cage. Mashed food and food pellets were placed on the cage bottom to encourage food uptake. The described lesion paradigm caused an extensive damage to the unilateral basal ganglia and the adjacent neocortex. For clinical scoring, mice were examined for forelimb flexion and body posture maintenance using the Bederson score $[$ as described in https://www.ahajournals.org/doi/pdf/10.1161/01.STR.17.3.472]. Animals were excluded from the studies when they fulfilled one of the following criteria: prolonged surgery time ( $>15 \mathrm{~min})$; no 
reflow after filament withdrawal; Clinical scoring of 0; seizures during/after MCAO or dead before experimental endpoint. A total of 7 animals were exluded ( 3 due to clinical scoring of 0 following MCAO and 4 due to seizures during/after MCAO).

\section{Cresyl violet staining}

Cresyl violet staining was performed $24 \mathrm{~h}$ after of reperfusion and infarct volume was assessed as percentage of the affected hemisphere. Five $20 \mu \mathrm{m}$ thick coronal sections taken at Bregma +2.8 , $+1.54,+0.14,-1.94$ and $-4.6 \mathrm{~mm}$, were stained with cresyl violet using vendor protocol and later digitalised using a Zeiss Axio Scan.Z1 at 5x magnification and lesions were determined using Zen Software (Zeiss). The person analysing was blinded to the treatment groups. Cerebral lesion volume was calculated summing up the volume of each section, while corrected for oedema (group numbers $>4)$.

\section{Immunofluorescence and Confocal Microscopy}

\section{Hippocampal organotypic slices}

$90 \mathrm{~min}$ after OGD induction, hippocampal slices were fixed for $1 \mathrm{~h}$ at room temperature in $4 \%$ paraformaldehyde in $0.1 \mathrm{M}$ phosphate buffer (PB), $\mathrm{pH}$ 7.4. Slices were then washed $5 \times$ in $0.1 \mathrm{M}$ $\mathrm{PB}$, permeabilized in $0.4 \%$ Triton $\mathrm{X}-100$ and blocked with $1.5 \%$ heat inactivated horse serum overnight at $4^{\circ} \mathrm{C} \cdot 1: 500$ anti-Gephyrin (Synaptic Systems) was incubated for 5 days at $4^{\circ} \mathrm{C}$ in permeabilizing buffer and washed $5 \times$ with $0.1 \mathrm{M}$ PB containing $1.5 \%$ heat inactivated horse serum. Slices were incubated for $3 \mathrm{~h}$ at room temperature with 1:250 anti-mouse DyLight 650 (Jackson ImmunoResearch, Burlington, ON, Canada) secondary antibody diluted in $0.1 \mathrm{M}$ PB containing $1.5 \%$ heat inactivated horse serum. Following $5 \times$ washes with $0.1 \mathrm{M}$ PB containing $1.5 \%$ heat- 
inactivated horse serum, slices were mounted with fluorescent mounting medium (DAKO, Mississauga, ON, Canada) onto microscope slides.

Slices were imaged using a Leica TCS SP2 scan head (Leica Microsystems) on a Leica DM6000 B upright microscope, equipped with HCX PL APO 63× NA 1.4 oil immersion objective using a $543 \mathrm{~nm}$ HeNe laser line. Image stacks were collected at $\mathrm{Z}=0.3 \mu \mathrm{m}$ and averaged 2-3 times to improve signal-to-noise ratio. For quantification, image stacks were obtained with identical parameters (laser intensity, filters, pinhole size, photomultiplier tube gain and offset). Representative images are maximum intensity projections of 5 sections from each stack.

\section{Immunohistochemical imaging of brain slices}

$24 \mathrm{~h}$ following MCAO, P60-70 (male or female) mice were anesthetized by intraperitoneal pentobarbital injection (Nembutal; $50 \mathrm{mg} / \mathrm{kg}$ ) and perfused transcardially with ice-cold oxygenated $\mathrm{ACSF}^{77}$, $\mathrm{pH} 7.4$, for $2 \mathrm{~min}$. Brains were immediately fixed in $4 \%$ PFA for $3 \mathrm{~h}$ at $4^{\circ} \mathrm{C}$. After rinsing in PBS, brains were incubated in $30 \%$ sucrose (in PBS) at $4^{\circ} \mathrm{C}$ over-night. $45 \mu \mathrm{m}-$ thick coronal sections were cut from frozen blocks using a sliding microtome (HM400; Microm) and stored at $-20^{\circ} \mathrm{C}$ in antifreeze solution. After 3 times 10 min washes in Tris-Triton Solution (50mM Tris, $150 \mathrm{mM} \mathrm{NaCl}, 0.05 \%$ Trito X-100, $\mathrm{pH} 7.4)$, sections were incubated in primary antibody solution (50mM Tris, $150 \mathrm{mM} \mathrm{NaCl}, 0.4 \%$ Triton X-100, 2\% normal goat serum, $\mathrm{pH} 7.4$ ) at $4^{\circ} \mathrm{C}$ overnight. Primary antibodies are listed in Table 1 . Sections were washed 3 x $10 \mathrm{~min}$ in TrisTriton Solution and incubated in secondary antibody solution (50mM Tris, $150 \mathrm{mM} \mathrm{NaCl,} 0.05 \%$ Triton X-100, 2\% normal goat serum, $\mathrm{pH} 7.4$ ) for 1 hour at room temperature with secondary antibodies raised in goat. All secondary antibodies used were diluted 1:500. Antibodies conjugated to AlexaFluor-488 and AlexaFluor-647 were purchased from Invitrogen, while antibodies 
conjugated to $\mathrm{Cy} 3$ were purchased from Jackson ImmunoResearch Laboratories. Sections were washed 3 times $10 \mathrm{~min}$ in Tris-Triton Solution and mounted on gelatin-coated slides using Fluorescence Mounting Medium (Dako). Z-stack images (4 optical sections, $0.75 \mu \mathrm{m}$ step size) were recorded of all sections using confocal laser scanning microscopy (LSM 700, Carl Zeiss). Images were taken using a 40x objective with a numerical aperture of 1.4, and pixel size of 112 $\mathrm{nm}^{2}$. Three juxtaposed images of the motor cortex layer $2 / 3$ were taken on the ipsi- and contralateral hemispheres. To reduce variability, multiple images were captured from 3 sections per mouse and total of 4-5 mice were analyzed per condition/genotype. Cluster density values were averaged from these sections. All imaging parameters were kept constant between MCAO and sham animals. For cluster analysis, a custom Python-script using the ImageJ image-processing framework was used. The script can be used as a plugin and is openly available on a github repository (https://github.com/dcolam/Cluster-Analysis-Plugin). Representative example images were processed using ImageJ. Statistical tests were performed using Prism software (GraphPad) using 5 mice per group.

\section{Microglia analysis}

Iba1 staining was acquired using a spinning-disk confocal microscope (Nikon Ti2 coupled to Yokogawa CSU-W1 confocal scanning unit) with a Omicron modified Light HUB+ laser emitting at $488 \mathrm{~nm}$ and a CFI Plan Apochromat Lambda 60X oil objective (N.A 1.40, W.D. 0.13mm). 3D stack images of $25.5 \mu \mathrm{m}$ were acquired with a z-step of $0.3 \mu \mathrm{m}$ in layer II/III of the motor cortex (2-3 slices per animal, 5 animals per group). For the microglia density analysis, individual microglial cells were counted in FIJI, through colocalization of the Ibal and DAPI stainings in 3D 
and the «Analyze Particles» function. The mean intensity and area covered by the Iba1 signal were analyzed on the maximum intensity projection of the stack in FIJI.

Imaris Software (Bitplane, Switzerland) was used for reconstruction of MCAO BDNFwt/wt

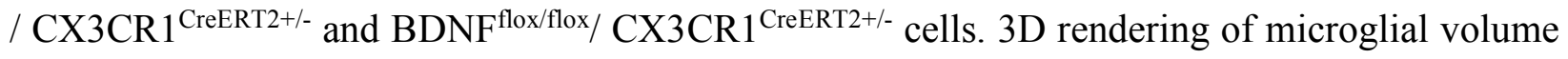
was based on Iba1 immunoreactivity, applying recorded algorithms with fixed thresholds for Iba1 signal intensity. Morphometric values were extracted per individual cells (BDNFwt/wt /

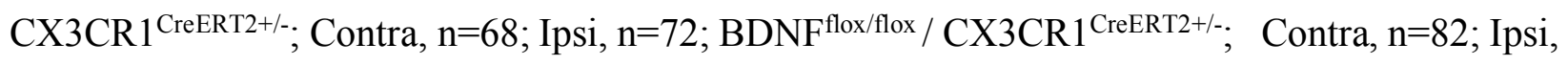
$\mathrm{n}=94)$.

\section{Dendrite Reconstructions, Spine Quantification, cluster Quantification}

3D confocal stacks were deconvolved with Huygens Essentials software (Scientific Volume Imaging, Hilversum, The Netherlands) using a full maximum likelihood extrapolation algorithm. Stacks were then imported and rendered using the Surpass function in Imaris software (Bitplane AG). Experimenter was blinded to conditions and treatment groups, spines were manually counted and using the ratio of the diameter and length of the head and neck of spines it was possible to distinguish between stubby, mushroom, and thin subtypes of dendritic spines. These classifications were based on previously established criteria ${ }^{34}$. Lastly, $n$ values for spine analysis represent $\sim 75-$ $100 \mu \mathrm{m}$ of dendrite from 1-2 cells imaged from each slice. The number and volume of gephyrin clusters were quantified using the Spot function of Imaris software, which differentiates puncta based on the fluorescence intensity.

\section{Electrophysiological Recordings and Analysis}


Slices were transferred into a temperature-controlled chamber $\left(25^{\circ} \mathrm{C}\right)$ mounted on an upright microscope (DM LFSA, Leica Microsystems) and continuously perfused with external solution. Patch recording electrodes were pulled from borosilicate glass (GC150TC; Clark Instruments, Old Sarum, Salisbury UK). All electrophysiological recordings were made using an Axopatch 200A amplifier (Molecular Devices, Sunnyvale, CA, U.S.A.).

AMPA-mediated mEPSCs were gathered from whole-cell voltage-clamp recordings of CA1 pyramidal neurons obtained at $25^{\circ} \mathrm{C}$ using electrodes with resistances of 4-5 $\mathrm{M} \Omega$ and filled with intracellular solution containing (in mM): K-Gluconate, 120; EGTA, 1; HEPES, 10; MgATP, 5; Na-GTP 0.5; NaCl, 5; KCl, 5; phosphocreatine, 10; 295 mOsm; pH adjusted with $\mathrm{KOH}$ to 7.3. $\mathrm{mEPSCs}$ were recorded at $-60 \mathrm{mV}$ and in the presence of $1 \mu \mathrm{M}$ tetrodotoxin (TTX), $15 \mu \mathrm{M}$ 3-[(R)-2-carboxypiperazin-4-yl]-propyl-1-phosphonic acid (CPP), $100 \mu \mathrm{M}$ picrotoxin, and $1 \mu \mathrm{M}$ CGP55845 in the external Tyrode's solution. Access resistance was monitored with brief test pulses at regular intervals (2-3 min) throughout the experiment. Access resistance was usually 10$13 \mathrm{M} \Omega$ and data were discarded if the resistance deviated more than $10 \%$ through the course of the experiment. Series resistance of the access pulse and decay time was also used for the calculation of total membrane capacitance. After the holding current had stabilized, data were recorded at a sampling frequency of $10 \mathrm{kHz}$ and filtered at $2 \mathrm{kHz}$ for 10 to $15 \mathrm{~min}$.

$\mathrm{GABA}_{\mathrm{A}} \mathrm{R}$-mediated mIPSCs were gathered from whole-cell voltage-clamp recordings of CA1 pyramidal neurons obtained at $25^{\circ} \mathrm{C}$ using electrodes with resistances of $4-5 \mathrm{M} \Omega$ and filled with intracellular solution containing (in $\mathrm{mM}$ ): $\mathrm{CsCl}, 140 ; \mathrm{NaCl}, 4 ; 0.5, \mathrm{CaCl} 2 ; \mathrm{HEPES}, 10$; EGTA, 5; QX-314, 2; Mg-ATP, 2; Na-GTP 0.5; 290 mOsm; pH adjusted with CsOH to 7.36. mIPSCs were recorded at $-60 \mathrm{mV}$ and in the presence of $1 \mu \mathrm{M}$ TTX, $25 \mu \mathrm{M} \mathrm{CPP}, 5 \mu \mathrm{M}$ CGP55845, $5 \mu \mathrm{m}$ 6-cyano-7-nitroquinoxaline-2,3-dione (CNQX) and $0.3 \mu \mathrm{m}$ strychnine in external Tyrode's 
solution. Access resistance was monitored with brief test pulses at regular intervals (2-3 min) throughout the experiment. After the holding current had stabilized, data were recorded at a sampling frequency of $10 \mathrm{kHz}$ and filtered at $2 \mathrm{kHz}$ for 10 to $15 \mathrm{~min}$.

All mEPSCs and mIPSCs were detected offline using the Mini Analysis Software (Synaptosoft, Decatur, GA, USA). The amplitude threshold for mEPSC and mIPSCs detection was set at four times the root-mean-square value of a visually event-free recording period. From every experiment, $5 \mathrm{~min}$ of stable recording was randomly selected for blinded analysis of amplitude and inter-event interval. The data obtained was then used to plot cumulative histograms with an equal contribution from every cell. For statistical analysis, data were averaged for every single cell. It should be noted that the amplitude analysis was conducted only on single mEPSCs and mIPSCs that did not have subsequent events occurring during their rising and decaying phases. For frequency analysis, all selected events were considered.

\section{Pharmacological Treatments}

To scavenge BDNF, slices were treated with TrkB-Fc (R\&D Systems; Minneapolis, MN, USA), a fusion protein in which the BDNF binding site of the TrkB receptor replaces the Fc fragment of a human IgG1 antibody. We found that TrkB-Fc treatment to hippocampal cultures for $24 \mathrm{~h}$ downregulated TrkB receptor phosphorylation (data not shown). TrkB-Fc was diluted in culture media at a final concentration of $10 \mu \mathrm{g} / \mathrm{ml}$ and treatment began immediately following induction of OGD. ERK activation was inhibited using $30 \mu \mathrm{M}$ of MEK inhibitor PD98059 (Tocris Biosciences, ON, Burlington, Canada), GSK3 $\beta$ activity was inhibited using $25 \mu \mathrm{M}$ GSK3 $\beta$-IX (Tocris Biosciences). PD98059 and GSK3 $\beta$-IX were diluted in dimethyl sulfoxide (Invitrogen) and treatment began overnight prior to OGD induction, removed during induction and continued 
for $90 \mathrm{~min}$ after. Control sister cultures were treated with control culture media containing

dimethyl sulfoxide only. The following function blocking antibodies were used: 1:200 anti-p75 (kind gift from Dr. P. Barker, McGill University, Montreal, QC, Canada; Rex antibody for more information see ${ }^{78}, 1: 200$ anti-proBDNF (kind gift from Dr. Philip Barker, McGill University, Montreal, Canada) and 1:100 anti-mBDNF (N-9, Developmental Studies Hybridoma Bank, University of Iowa, IA, USA; for more information see ${ }^{79}$. Function blocking antibody treatment began $2 \mathrm{~h}$ prior to OGD induction, removed during induction and continued for $90 \mathrm{~min}$ after.

\section{Biolistic Gene Transfection}

Cartridges were prepared according to manufacturer's protocol (Bio-Rad, Helios Gene Gun). Briefly, $15 \mathrm{mg}$ of gold particles $(1 \mu \mathrm{m}$ diameter $)$ were first coated with $0.05 \mathrm{M}$ spermidine. $15 \mu \mathrm{g}$ of plasmid DNA expressing tdTomato and $45 \mu \mathrm{g}$ of wildtype gephyrin-GFP (gephyrinwT-GFP) or dephosphorylation mutant gephyrin-GFP S268A/S270A (gephyrin $2268 \mathrm{~A} / \mathrm{S2} 20 \mathrm{~A}-\mathrm{GFP}$ ). Plasmids were then precipitated onto the particles by adding $\mathrm{CaCl} 2$. The coated particles were resuspended into $100 \%$ ethanol and infused into Tefzel tubing, which were then coated with the particles. Coated tubing was cut into 0.5 inch cartridges which were then transfected into mature organotypic slice cultures by shooting at a distance of $2 \mathrm{~cm}$ with a pressure of $200 \mathrm{psi}$ through a nylon mesh. Following $48 \mathrm{~h}$, slices which expressed target plasmids in CA1 pyramidal neurons were processed with OGD or control Tyrode solution.

\section{Western blotting}

$24 \mathrm{~h}$ following MCAO, mice were killed by decapitation and brains were dissected on ice. The ipsi- and contralateral cortices were immediately transferred to lysis buffer (50 mM Tris, $\mathrm{pH} 7.6$, 
$150 \mathrm{mM} \mathrm{NaCl}, 1 \%$ Triton X-100, CompleteMini Protease Inhibitor Mixture, Roche). The cortices were homogenized and incubated on ice for 1 hour. Lysates were centrifuged at 20,000 RPM for $30 \mathrm{~min}$ at $4^{\circ} \mathrm{C}$, and supernatants were stored at $-80^{\circ} \mathrm{C}$. Samples were run on Tris-glycine polyacrylamide gels and proteins were transferred to PVDF membranes. Primary antibodies (see Table 1) were incubated in Tris-buffered saline with $0.05 \%$ Tween 20 (TBST), including 5\% WesternBlocking Solution (Roche) overnight at $4^{\circ} \mathrm{C}$. Membranes were washed $5 \mathrm{x} 5 \mathrm{~min}$ in TBST. HRP-coupled donkey secondary antibodies $(1: 30,000)$ and fluorescent-coupled donkey secondary antibodies $(1: 20,000)$ were incubated for $30 \mathrm{~min}$ at room temperature, and membranes were washed again 5 times 5 min in TBST. Fluorescent signals were captured using the Odyssey® CLx Imager. SuperSignal West Pico ChemiluminescentSubstrate (Thermo Fisher Scientific) was applied to visualize HRP labelled antibodies and developed using the FUJIFILM Luminescent Image Analyzer LAS-1000 plus \& Intelligent Dark Box II (Fujifilm). Images were analyzed using ImageJ and statistical tests were performed using Prism software (GraphPad) using a minimum of 4 mice per group. Western blot membrane stripping for restaining was performed for p-ERK1/2 and ERK1/2 antibodies using a mild stripping protocol from abcam. Briefly, membranes were incubated twice for 5-10 min with mild stripping buffer (200mM gylcine, 20mM SDS, 0.01\% Tween 20, pH 2.2) followed by 2 x 10min incubation with PBS and 2 x 5 min incubations with TBST. Efficiency of stripping was checked by incubating with chemiluniscent detection. When stripping was judged satisfactory, the membranes were rinsed and incubated with primary antibody.

\section{Statistical Analysis}


An a priori power analysis was conducted using data reported in Figures 5-8 obtained with the MCAO surgery procedure. Combined with type-1 error set to 0.05 . Power set to 0.8 (1-beta-error), we determined the effect size with a group size of 5 . These indicated an inter individual variation (SD) of $10-15 \%$. We used multiple group comparison test (Bonferroni) to make maximal number of comparisons between groups.

For the slice culture comparisons we performed 2-way ANOVA and independent t-tests on the following figures: Figure 2b, d, e, ,f', f, g, g'; Figure 3b, c, d, f, g, h; Figure 4b, c, d, g, h, i. The t-tests were performed to confirm significance between conditions as 2-way ANOVA and post hoc test does not cover all the comparisons of our interest. These t-test $\mathrm{p}$ values are unprotected, therefore we adjusted them using Bonferroni correction (e.g. if the set of the data is compared 5 times, we need to multiply the p-value by 5). The listed significant comparisons all have protected p-values by Bonferroni post-test. $* * * \mathrm{p}<0.001 ; * * \mathrm{p}<0.01 ;{ }^{*} \mathrm{p}<0.05$.

Comparison between two groups were made using two-tailed independent Student's $t$-test. Comparisons between multiple groups and treatments were made using two-way ANOVA with post hoc Bonferroni multiple comparison test. Comparisons between multiple groups were made using one-way ANOVA. Cumulative probability plots were compared using KolmogorovSmirnov test for probability distributions. Results are expressed as mean $\pm \mathrm{sd}$.

\section{Author Contributions}

SKT and RAM designed the study. ZST and PKYC performed and analyzed the OGD electrophysiology. RG performed and analysed the morphology data from OGD experiments. ZST, PKYC and RG supported data collection and analysis. MV performed the MCAO, TC, MV, FM, RP and SS performed and analysed the mouse MCAO data. DC wrote the macro for 
morphology analysis. SBN performed data analysis. RCP, PAB, SAB and JK provided technical expertise, infrastructure support and biological reagents for this study. RG, PKYC, ZST, FM prepared OGD figures, TC, SS, SBN, MV, FM and SKT prepared MCAO figures. All authors contributed to writing and editing the manuscript.

\section{Acknowledgments:}

We thank Dr. J-M. Fritschy for antibody reagents and feedback on the manuscript; F. Charron for preparation and maintenance of organotypic slice cultures; G.Bosshard for preparation of microglia cultures; Plexxikon for sharing their PLX5622 chow formulated compound for our studies. This project was initiated as part of ZNZ-McGill-Oxford tripartite collaboration. This work was supported by grants from CIHR to RAM (grant ID: MOP 133611), Savoy Foundation to RAM, Swiss National Science Foundation (320030_179277), ERA-NET NEURON (32NE30_173678/1), the Synapsis Foundation and the Vontobel foundation to JK, Swiss National

Science Foundation grants to SKT (31003A_159867, 310030_192522), internal UZH funding to SKT, Forschungskredit Candoc grant to TC and Forschungskredit postdoc grant to MV.

\section{Competing financial interests}

The authors declare no competing financial interests. 


\section{References}

1. Donkor, E. S. Stroke in the 21st Century: A Snapshot of the Burden, Epidemiology, and Quality of Life. Stroke Res Treat 2018, 1-10 (2018).

2. Brouns, R. \& Deyn, P. P. D. The complexity of neurobiological processes in acute ischemic stroke. Clin Neurol Neurosur 111, 483-495 (2009).

3. Lakhan, S. E., Kirchgessner, A. \& Hofer, M. Inflammatory mechanisms in ischemic stroke: therapeutic approaches. J Transl Med 7, 97 (2009).

4. Albers, G. W., Goldstein, L. B., Hall, D., Lesko, L. M. \& Investigators, for the A. A. S. Aptiganel Hydrochloride in Acute Ischemic Stroke. Jama 286, 2673 (2001).

5. Lyden, P. D., Jackson-Friedman, C., Shin, C. \& Hassid, S. Synergistic Combinatorial Stroke Therapy: A Quantal Bioassay of a GABA Agonist and a Glutamate Antagonist. Exp Neurol 163, 477-489 (2000).

6. Zhang, J., Liu, J., Li, D., Zhang, C. \& Liu, M. Calcium antagonists for acute ischemic stroke. Cochrane Database Syst Rev 2, CD001928 (2019).

7. Gibson, L. M., Brazzelli, M., Thomas, B. M. \& Sandercock, P. A. G. A systematic review of clinical trials of pharmacological interventions for acute ischaemic stroke (1955-2008) that were completed, but not published in full. Trials 11, 43 (2010).

8. Shuaib, A. et al. NXY-059 for the Treatment of Acute Ischemic Stroke. New Engl J Med 357, 562-571 (2007).

9. Campbell, B. C. V. et al. Ischaemic stroke. Nat Rev Dis Primers 5, 70 (2019).

10. Zhang, S., Boyd, J., Delaney, K. \& Murphy, T. H. Rapid Reversible Changes in Dendritic Spine Structure In Vivo Gated by the Degree of Ischemia. J Neurosci 25, 5333-5338 (2005).

11. Alicke, B. \& Schwartz-Bloom, R. D. Rapid Down-Regulation of GABAA Receptors in the Gerbil Hippocampus Following Transient Cerebral Ischemia. J Neurochem 65, 2808-2811 (2002).

12. Yu, S.-J. et al. Local administration of AAV-BDNF to subventricular zone induces functional recovery in stroke rats. Plos One 8, e81750 (2013).

13. Schäbitz, W.-R. et al. Intravenous Brain-Derived Neurotrophic Factor Enhances Poststroke Sensorimotor Recovery and Stimulates Neurogenesis. Stroke 38, 2165-2172 (2007).

14. Jiang, Y. et al. Effects of brain-derived neurotrophic factor on local inflammation in experimental stroke of rat. Mediat Inflamm 2010, 372423 (2010).

15. Gottlieb, M. et al. Brain-derived neurotrophic factor blood levels in two models of transient brain ischemia in rats. Gen Physiol Biophys 32, 139-142 (2013). 
16. Adachi, N., Numakawa, T., Richards, M., Nakajima, S. \& Kunugi, H. New insight in expression, transport, and secretion of brain-derived neurotrophic factor: Implications in brainrelated diseases. World J Biological Chem 5, 409-28 (2014).

17. Yang, J. et al. proBDNF negatively regulates neuronal remodeling, synaptic transmission, and synaptic plasticity in hippocampus. Cell Reports 7, 796-806 (2014).

18. Tanaka, J.-I. et al. Protein synthesis and neurotrophin-dependent structural plasticity of single dendritic spines. Sci New York N Y 319, 1683-7 (2008).

19. Kraemer, B. R., Yoon, S. O. \& Carter, B. D. Handb Exp Pharmacol 220, 121-164 (2014).

20. Brünig, I., Penschuck, S., Berninger, B., Benson, J. A. \& Fritschy, J. M. BDNF reduces miniature inhibitory postsynaptic currents by rapid downregulation of GABAA receptor surface expression. European Journal of Neuroscience 13, 1320-1328 (2001).

21. Mou, L., Dias, B. G., Gosnell, H. \& Ressler, K. J. Gephyrin plays a key role in BDNFdependent regulation of amygdala surface GABAARs. Neuroscience 255, 33-44 (2013).

22. Wood, H. A role for double-negative T cells in post-stroke neuroinflammation. Nat Rev Neurol 15, 246-247 (2019).

23. Schafer, D. P. \& Stevens, B. Microglia Function in Central Nervous System Development and Plasticity. Csh Perspect Biol 7, a020545 (2015).

24. Lenz, K. M. \& Nelson, L. H. Microglia and Beyond: Innate Immune Cells As Regulators of Brain Development and Behavioral Function. Front Immunol 9, 698 (2018).

25. Lenz, K. M., Nugent, B. M., Haliyur, R. \& McCarthy, M. M. Microglia are essential to masculinization of brain and behavior. The Journal of neuroscience : the official journal of the Society for Neuroscience 33, 2761-2772 (2013).

26. Reemst, K., Noctor, S. C., Lucassen, P. J. \& Hol, E. M. The Indispensable Roles of Microglia and Astrocytes during Brain Development. Front Hum Neurosci 10, 566 (2016).

27. Fumagalli, S., Perego, C., Ortolano, F. \& Simoni, M.-G. D. CX3CR1 deficiency induces an early protective inflammatory environment in ischemic mice. Glia 61, 827-42 (2013).

28. Li, Y., Du, X.-F., Liu, C.-S., Wen, Z.-L. \& Du, J.-L. Reciprocal regulation between resting microglial dynamics and neuronal activity in vivo. Dev Cell 23, 1189-202 (2012).

29. Cserép, C. et al. Microglia monitor and protect neuronal function through specialized somatic purinergic junctions. Sci New York N Y 367, 528-537 (2019).

30. Chen, Z. et al. Lipopolysaccharide-induced microglial activation and neuroprotection against experimental brain injury is independent of hematogenous TLR4. J Neurosci Official J Soc Neurosci 32, 11706-15 (2012).

31. Jang, S.-W. et al. A selective TrkB agonist with potent neurotrophic activities by 7,8dihydroxyflavone. P Natl Acad Sci Usa 107, 2687-92 (2010). 
32. Gill, R., Chang, P. K.-Y., Prenosil, G. A., Deane, E. C. \& McKinney, R. A. Blocking brainderived neurotrophic factor inhibits injury-induced hyperexcitability of hippocampal CA3 neurons. Eur J Neurosci 38, 3554-3566 (2013).

33. Bergeron, M., Yu, A. Y., Solway, K. E., Semenza, G. L. \& Sharp, F. R. Induction of hypoxia-inducible factor-1 (HIF-1) and its target genes following focal ischaemia in rat brain. Eur J Neurosci 11, 4159-4170 (1999).

34. McKinney, R. A. Excitatory amino acid involvement in dendritic spine formation, maintenance and remodelling. J Physiology 588, 107-16 (2009).

35. Lai, S.-W. et al. Regulatory Effects of Neuroinflammatory Responses Through BrainDerived Neurotrophic Factor Signaling in Microglial Cells. Mol Neurobiol 55, 7487-7499 (2018).

36. Béjot, Y. et al. Time-dependent contribution of non neuronal cells to BDNF production after ischemic stroke in rats. Neurochem Int 58, 102-11 (2010).

37. Mohammadi, A., Amooeian, V. G. \& Rashidi, E. Dysfunction in Brain-Derived Neurotrophic Factor Signaling Pathway and Susceptibility to Schizophrenia, Parkinson's and Alzheimer's Diseases. Curr Gene Ther 18, 45-63 (2018).

38. Tyagarajan, S. K. et al. Regulation of GABAergic synapse formation and plasticity by GSK3beta-dependent phosphorylation of gephyrin. Proceedings of the National Academy of Sciences 108, 379-384 (2011).

39. Tyagarajan, S. K. et al. Extracellular signal-regulated kinase and glycogen synthase kinase $3 \beta$ regulate gephyrin postsynaptic aggregation and GABAergic synaptic function in a calpaindependent mechanism. The Journal of biological chemistry 288, 9634-9647 (2013).

40. Morris, G. P. et al. A Comparative Study of Variables Influencing Ischemic Injury in the Longa and Koizumi Methods of Intraluminal Filament Middle Cerebral Artery Occlusion in Mice. Plos One 11, e0148503 (2016).

41. Yassi, N. et al. The Association between Lesion Location and Functional Outcome after Ischemic Stroke. Int J Stroke 10, 1270-1276 (2015).

42. Zhou, L. et al. Treatment of cerebral ischemia by disrupting ischemia-induced interaction of nNOS with PSD-95. Nat Med 16, 1439-43 (2010).

43. Parkhurst, C. N. et al. Microglia Promote Learning-Dependent Synapse Formation through Brain-Derived Neurotrophic Factor. Cell 155, 1596-1609 (2013).

44. Elmore, M. R. P. et al. Replacement of microglia in the aged brain reverses cognitive, synaptic, and neuronal deficits in mice. Aging Cell 17, e12832 (2018).

45. Spangenberg, E. E. et al. Eliminating microglia in Alzheimer's mice prevents neuronal loss without modulating amyloid- $\beta$ pathology. Brain J Neurology 139, 1265-81 (2016).

46. Elmore, M. R. P. et al. Colony-stimulating factor 1 receptor signaling is necessary for microglia viability, unmasking a microglia progenitor cell in the adult brain. Neuron 82, 380-97 (2014). 
47. Calabrese, F. et al. Brain-derived neurotrophic factor: a bridge between inflammation and neuroplasticity. Front Cell Neurosci 8, 430 (2014).

48. Inoue, K. \& Tsuda, M. Microglia in neuropathic pain: cellular and molecular mechanisms and therapeutic potential. Nat Rev Neurosci 19, 138-152 (2018).

49. Paolicelli, R. C. et al. TDP-43 Depletion in Microglia Promotes Amyloid Clearance but Also Induces Synapse Loss. Neuron 95, 297-308.e6 (2017).

50. Rajan, W. D. et al. Dissecting functional phenotypes of microglia and macrophages in the rat brain after transient cerebral ischemia. Glia 67, 232-245 (2018).

51. Gomes, C. et al. Activation of microglial cells triggers a release of brain-derived neurotrophic factor (BDNF) inducing their proliferation in an adenosine A2A receptor-dependent manner: A2A receptor blockade prevents BDNF release and proliferation of microglia. $J$ Neuroinflamm 10, 16 (2013).

52. Wei, Z. et al. Notch Activation Enhances the Microglia-Mediated Inflammatory Response Associated With Focal Cerebral Ischemia. Stroke 42, 2589-2594 (2011).

53. Buscemi, L., Price, M., Bezzi, P. \& Hirt, L. Spatio-temporal overview of neuroinflammation in an experimental mouse stroke model. Sci Rep-uk 9, 507 (2019).

54. Clarkson, A. N., Huang, B. S., Macisaac, S. E., Mody, I. \& Carmichael, S. T. Reducing excessive GABA-mediated tonic inhibition promotes functional recovery after stroke. Nature 468, 305-9 (2010).

55. Dejanovic, B. \& Schwarz, G. Neuronal nitric oxide synthase-dependent S-nitrosylation of gephyrin regulates gephyrin clustering at GABAergic synapses. The Journal of neuroscience: the official journal of the Society for Neuroscience 34, 7763-7768 (2014).

56. Je, H. S. et al. ProBDNF and mature BDNF as punishment and reward signals for synapse elimination at mouse neuromuscular junctions. J Neurosci Official J Soc Neurosci 33, 9957-62 (2013).

57. Woo, N. H. et al. Activation of $\mathrm{p} 75 \mathrm{NTR}$ by proBDNF facilitates hippocampal long-term depression. Nat Neurosci 8, 1069-1077 (2005).

58. Kaplan, D. R. \& Miller, F. D. Neurotrophin signal transduction in the nervous system. Curr Opin Neurobiol 10, 381-391 (2000).

59. Lin, P.-Y., Kavalali, E. T. \& Monteggia, L. M. Genetic Dissection of Presynaptic and Postsynaptic BDNF-TrkB Signaling in Synaptic Efficacy of CA3-CA1 Synapses. Cell Reports 24, 1550-1561 (2018).

60. Hedrick, N. G. et al. Rho GTPase complementation underlies BDNF-dependent homo- and heterosynaptic plasticity. Nature 538, 104-108 (2016).

61. Sánchez-Huertas, C. \& Rico, B. CREB-Dependent Regulation of GAD65 Transcription by BDNF/TrkB in Cortical Interneurons. Cereb Cortex New York N Y 1991 21, 777-88 (2010).

62. Ferrini, F. \& Koninck, Y. D. Microglia control neuronal network excitability via BDNF signalling. Neural Plast 2013, 429815 (2013). 
63. Sorge, R. E. et al. Different immune cells mediate mechanical pain hypersensitivity in male and female mice. Nat Neurosci 18, 1081-3 (2015).

64. Beggs, S., Trang, T. \& Salter, M. W. P2X4R+ microglia drive neuropathic pain. Nat Neurosci 15, 1068-73 (2012).

65. Masuda, T. et al. Transcription factor IRF5 drives P2X4R+-reactive microglia gating neuropathic pain. Nat Commun 5, 3771 (2014).

66. Tsuda, M. et al. IFN-gamma receptor signaling mediates spinal microglia activation driving neuropathic pain. P Natl Acad Sci Usa 106, 8032-7 (2009).

67. Ohsawa, K. et al. Involvement of P2X 4 and $\mathrm{P} 2 \mathrm{Y} 12$ receptors in ATP-induced microglial chemotaxis. Glia 55, 604-616 (2007).

68. Monif, M., Reid, C. A., Powell, K. L., Smart, M. L. \& Williams, D. A. The P2X7 Receptor Drives Microglial Activation and Proliferation: A Trophic Role for P2X7R Pore. J Neurosci 29, 3781-3791 (2009).

69. Gan, Y. et al. Ischemic neurons recruit natural killer cells that accelerate brain infarction. $P$ Natl Acad Sci Usa 111, 2704-9 (2014).

70. Chen, Z. et al. Microglial displacement of inhibitory synapses provides neuroprotection in the adult brain. Nat Commun 5, 4486 (2014).

71. Guérineau, N. C., McKinney, R. A., Debanne, D., Mollard, P. \& Gähwiler, B. H. Organotypic cultures of the rat anterior pituitary: morphology, physiology and cell-to-cell communication. J Neurosci Meth 73, 169-176 (1997).

72. Paola, V. D., Arber, S. \& Caroni, P. AMPA receptors regulate dynamic equilibrium of presynaptic terminals in mature hippocampal networks. Nat Neurosci 6, 491-500 (2003).

73. Gee, C. E. et al. NMDA receptors and the differential ischemic vulnerability of hippocampal neurons. European Journal of Neuroscience 23, 2595-2603 (2006).

74. Yona, S. et al. Fate mapping reveals origins and dynamics of monocytes and tissue macrophages under homeostasis. Immunity 38, 79-91 (2012).

75. Rios, M. et al. Conditional Deletion Of Brain-Derived Neurotrophic Factor in the Postnatal Brain Leads to Obesity and Hyperactivity. Mol Endocrinol 15, 1748-1757 (2001).

76. Vaas, M., Ni, R., Rudin, M., Kipar, A. \& Klohs, J. Extracerebral Tissue Damage in the Intraluminal Filament Mouse Model of Middle Cerebral Artery Occlusion. Front Neurol 8, 85 (2017).

77. Notter, T., Panzanelli, P., Pfister, S., Mircsof, D. \& Fritschy, J.-M. A protocol for concurrent high-quality immunohistochemical and biochemical analyses in adult mouse central nervous system. The European journal of neuroscience 39, 165-175 (2014).

78. Weskamp, G. \& Reichardt, L. F. Evidence that biological activity of NGF is mediated through a novel subclass of high affinity receptors. Neuron 6, 649-663 (1991). 
bioRxiv preprint doi: https://doi.org/10.1101/2020.04.22.055087; this version posted December 20, 2020. The copyright holder for this preprint (which was not certified by peer review) is the author/funder, who has granted bioRxiv a license to display the preprint in perpetuity. It is made available under aCC-BY-ND 4.0 International license.

79. Kolbeck, R., Bartke, I., Eberle, W. \& Barde, Y.-A. Brain-Derived Neurotrophic Factor Levels in the Nervous System of Wild-Type and Neurotrophin Gene Mutant Mice. J Neurochem 72, 1930-1938 (2008). 


\section{Figure Legends:}

\section{Figure 1 -OGD induces morphological and functional deficits in excitatory synapses.}

(a) Example tertiary dendrites from CA1 pyramidal neurons expressing myristoylated-eGFP in control versus $90 \mathrm{~min}$ post-OGD organotypic hippocampal cultures. Scale $=2 \mu \mathrm{m}$. (b) Quantification of dendritic spines categorized into stubby, mushroom and long thin subtypes $\left({ }^{*} p\right.$ $<0.05$ and ${ }^{* *} p<0.01$, two-tailed independent Student's $t$-test). Total spine density (spines/ $\mu$ m of dendrite): Control $-1.76 \pm 0.08(n=8)$; OGD $-1.25 \pm 0.11(n=8)$. (c) Example images of maximum intensity projections of area CA1 immunostained for gephyrin and VGAT in control cultures versus 90 min following OGD. (d) Quantification of number of gephyrin clusters per confocal stack (consisting of five 512x512 pixel z-planes each; ***p $<0.0001$, two-tailed independent Student's $t$-test; gephyrin cluster values were normalized to control). Mean number (A.U.): Control $-1.00 \pm 0.03$ ( $n=10$ slices); OGD $-0.40 \pm 0.05$ ( $n=10$ slices). (e) Quantification of the total volume of gephyrin cluster $(* * * p<0.0001$, two-tailed independent Student's $t$-test). Mean volume (A.U.): Control $-1.00 \pm 0.05$; OGD $-0.53 \pm 0.03$. Data shown as mean \pm s.d. $(n=30$ cells). (f) Cumulative probability histogram and mean \pm SEM amplitude $(p<0.001$, KolmogorovSmirnov test). (f') Cumulative probability histogram and mean \pm SEM for IEIs of mEPSCs $(p<$ 0.05, Kolmogorov-Smirnov test). (f'') sample mEPSC traces from control cells and OGD cells. (g) Cumulative probability histogram and mean \pm SEM amplitude of mIPSCs $(p<0.001$, Kolmogorov-Smirnov test). (g') Cumulative probability histogram and mean \pm SEM for IEIs of mIPSCs $(p<0.05$, Kolmogorov-Smirnov test). (f'') sample mEPSC traces from control cells and OGD cells. Data shown as mean \pm s.d.

Figure 2 - Scavenging BDNF rescues $O G D$-induced synapse deficits 
(a) Example dendrites from CA1 neurons expressing myristoylated-eGFP with and without TrkBfc treatment. Scale $=2 \mu \mathrm{m}$. (b) Quantification of dendritic spines $(* p<0.05$, Two-way ANOVA with Bonferroni multiple comparison test). Total spine density (spines/ $\mu \mathrm{m}$ of dendrite): Control $1.22 \pm 0.05(n=16) ;$ Control+TrkB-Fc - $1.40 \pm 0.10(n=12) ;$ OGD $-0.95 \pm 0.06(n=12)$; OGD+TrkB-Fc $-1.19 \pm 0.07(n=14)$. (b') Immunoprecipitation using TrkB-Fc chimera and WB against proBDNF or mBDNF. (c) Example images of maximum intensity projections of organotypic hippocampal slices immunostained for gephyrin with and without TrkB-Fc treatment. Scale $=2 \mu \mathrm{m}$. (d) Quantification of number of gephyrin puncta per confocal stack (consisting of five $512 \times 512$ pixel z-planes each; $* * * p<0.0001$, Two-way ANOVA with Bonferroni multiple comparison test; all gephyrin values were normalized to control). Mean number (A.U.): Control $1.00 \pm 0.04(n=15) ;$ Control+TrkB-Fc $-0.95 \pm 0.04(n=9$ slices $) ;$ OGD $-0.27 \pm 0.03(n=13)$; OGD+TrkB-Fc $-0.86 \pm 0.05(n=13)$. (e) Quantification of gephyrin puncta volume $(* * * p<$ 0.0001, Two-way ANOVA with Bonferroni multiple comparison test). Mean volume (A.U.): Control $-1.00 \pm 0.03$; Control+TrkB-Fc $-0.98 \pm 0.03 ;$ OGD $-0.67 \pm 0.06 ;$ OGD+TrkB-Fc -1.02 \pm 0.04 . Data shown as mean \pm s.d. ( $n=30$ cells). (f) Cumulative probability histogram of mean amplitude ( $p<0.001$, Two-way ANOVA with Bonferroni multiple comparison test). (f') Cumulative probability histogram for IEIs of mEPSCs $(p<0.05$, Two-way ANOVA with Bonferroni multiple comparison test). (g) Cumulative probability histogram of mean amplitude of mIPSCs ( $p>0.05$, Two-way ANOVA with Bonferroni multiple comparison test). (g') Cumulative probability histogram for IEIs of mIPSCs $(p<0.05$, Two-way ANOVA with Bonferroni multiple comparison test). Data shown as mean \pm s.d. 
Figure 3 - Differential proBDNF and mBDNF signaling induce synapse loss after ischemia.

(a) Example tertiary dendrites from CA1 pyramidal neurons of organotypic hippocampal slices immunostained for gephyrin, with or without anti-proBDNF or anti-p $75^{\mathrm{NTR}}(\mathrm{Rex})$ treatment before OGD. Scale $=2 \mu \mathrm{m}$. (b) Dendritic spine quantification $(* * * p<0.0001$ Two-way ANOVA with Bonferroni multiple comparison test). Total spine density (spines/ $\mu \mathrm{m}$ of dendrite): Control -1.43 $\pm 0.04(n=15) ;$ Control+anti-proBDNF $-1.37 \pm 0.07(n=15) ;$ Control+anti-p75 NTR $-1.42 \pm 0.05$ $(n=15) ;$ OGD $-0.95 \pm 0.04(n=39) ;$ OGD +anti-proBDNF $-1.40 \pm 0.03(n=30)$; OGD + anti$\mathrm{p} 75^{\mathrm{NTR}}-1.35 \pm 0.04(n=32)$. (c) Quantification of number of gephyrin cluster density (consisting of five 512x512 pixel z-planes each; $p=0.28$, Two-way ANOVA with Bonferroni multiple comparison test, compared to control and treated controls; all gephyrin values were normalized to control). Mean number (A.U.): Control - $1.00 \pm 0.03$ ( $n=18$ slices); Control+anti-proBDNF $0.97 \pm 0.07(n=6) ;$ Control+anti-p $75^{\mathrm{NTR}}-0.97 \pm 0.06(n=6) ;$ OGD $-0.43 \pm 0.03(n=20$ slices $)$; OGD + anti-proBDNF $-0.47 \pm 0.03$ ( $n=16$ slices $)$; OGD + anti-p75 $5^{\mathrm{NTR}}-0.47 \pm 0.04(n=9)$. (d) Quantification of gephyrin puncta volume ( $p=0.57$, Two-way ANOVA with Bonferroni multiple comparison test, compared to control and treated controls). Mean volume (A.U.): Control - 1.00 \pm 0.04 ; Control+anti-proBDNF $-0.95 \pm 0.07$; Control+anti-p75 ${ }^{\mathrm{NTR}}-1.01 \pm 0.09 ;$ OGD $-0.63 \pm$ 0.03; OGD+anti-proBDNF $-0.65 \pm 0.02$; OGD + anti-p $75^{\mathrm{NTR}}-0.62 \pm 0.03$. (e) Example tertiary dendrites from CA1 pyramidal neurons of organotypic hippocampal slices immunostained for gephyrin, with or without anti-mBDNF (N-9) treatment before OGD. Scale $=2 \mu \mathrm{m}$. (f) Quantification of dendritic spines $(* * p<0.001$ Two-way ANOVA with Bonferroni multiple comparison test). Total spine density (spines/ $\mu \mathrm{m}$ of dendrite): Control $-1.45 \pm 0.04(n=34)$; Control+anti-mBDNF (N-9) - $1.37 \pm 0.03(n=17)$; OGD - $0.95 \pm 0.05(n=31)$; OGD+anti-N-9 $-1.05 \pm 0.06$ ( $\mathrm{n}>20$ cells). (g) Quantification of number of gephyrin clusters per confocal stack; 
consisting of five $512 \times 512$ pixel z-planes each $(* * * p<0.0001$, Two-way ANOVA with Bonferroni multiple comparison test; all gephyrin values were normalized to control). Mean number (A.U.): Control $-1.00 \pm 0.03(n=11)$; Control+N-9 $-0.99 \pm 0.04(n=6$ slices $)$; OGD $0.44 \pm 0.04(n=12)$; OGD+ anti-mBDNF $(\mathrm{N}-9)-0.85 \pm 0.06(n=8)$. (h) Quantification of gephyrin cluster volume $(* * * p<0.0001$, Two-way ANOVA with Bonferroni multiple comparison test). Mean volume (A.U.): Control - $1.00 \pm 0.06$; Control+N-9 - $1.07 \pm 0.06$; OGD - 0.66 \pm 0.05 ; OGD $+\mathrm{N}-9-0.88 \pm 0.05$. Data shown as mean \pm s.d.

Figure $4-E R K 1 / 2$ and GSK3ß pathways induce gephyrin degradation but not dendritic spine

\section{loss after OGD.}

(a) Example tertiary dendrites from CA1 pyramidal neurons from control and 90 min post-OGD slices, immunostained for gephyrin, with or without pharmacological treatment (GSK3 $\beta$ inhibitor: GSK3 $\beta$-IX and MEK inhibitor: PD98059). Scale $=2 \mu \mathrm{m}$. (b) Dendritic spine quantification $(* * p$ $<0.01$ Two-way ANOVA with Bonferroni multiple comparison test). Total spine density (spines/ $\mu \mathrm{m}$ of dendrite): Control $-1.41 \pm 0.06(n=21)$; Control+PD+G.-IX $-1.46 \pm 0.07(n=19)$; OGD $-1.09 \pm 0.06(n=22)$; OGD $+\mathrm{PD}+\mathrm{G} .-\mathrm{IX}-1.15 \pm 0.05(n=21)$. (c) Quantification of number of gephyrin cluster density (consisting of five $512 \times 512$ pixel z-planes each; $* * * p<0.0001$, Twoway ANOVA with Bonferroni multiple comparison test; all gephyrin values were normalized to control). (d) Quantification of number of gephyrin cluster volume. Mean number (A.U.): Control $-1.00 \pm 0.08(n=11)$; Control+PD+G.-IX - $1.12 \pm 0.08(n=9) ;$ OGD - $0.44 \pm 0.08(n=9)$; OGD+PD+G.-IX $-1.16 \pm 0.09(n=9)$. (d) Quantification of gephyrin puncta volume $(* * p<0.001$ Two-way ANOVA with Bonferroni multiple comparison test). Mean volume (A.U.): Control $1.00 \pm 0.04 ;$ Control+PD+G.-IX $-0.91 \pm 0.05 ;$ OGD $-0.67 \pm 0.04 ;$ OGD+PD+G.-IX $-0.96 \pm$ 
0.07. (e) Maximum intensity projection of example CA1 pyramidal neurons which had been biolistitally transfected with a plasmid expressing tdTomato. Scale $=10 \mu \mathrm{m}$. Neurons were cotransfected with either wildtype or mutant gephyrin. (f) Example tertiary dendrites from CA1 pyramidal neurons from control and 90 min post-OGD slices which were transfected with either

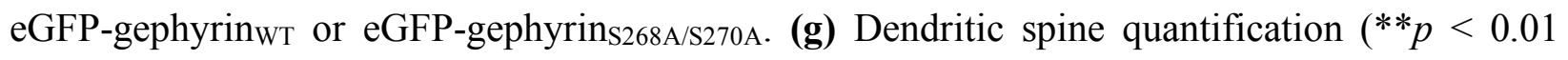
Two-way ANOVA with Bonferroni multiple comparison test). Total spine density (spines/ $\mu \mathrm{m}$ of dendrite): Control + gephyrin ${ }_{W T}-G F P-1.05 \pm 0.052(n=11)$; Control + eGFP-gephyrin ${ }_{S 268 \mathrm{~A} / \mathrm{S} 270 \mathrm{~A}}$ $-1.05 \pm 0.090(n=12)$; OGD - eGFP-gephyrin ${ }_{W T} 0.74 \pm 0.056(n=14) ;$ OGD + eGFPgephyrin $_{\mathrm{S} 268 \mathrm{~A} / \mathrm{S} 270 \mathrm{~A}}-0.69 \pm 0.064(n=8) ;{ }^{* *} p<0.01$, Two-way ANOVA with Bonferroni multiple comparison test). (h) Quantification of number of gephyrin cluster density ( $* * p<0.01$, Two-way ANOVA with Bonferroni multiple comparison test). Density: Control + eGFP-gephyrinwt -0.38 \pm 0.58; Control + eGFP-gephyrin ${ }_{\mathrm{S} 268 \mathrm{~A} / \mathrm{S} 270 \mathrm{~A}}-0.47 \pm 0.092 ;$ OGD + eGFP-gephyrin ${ }_{\mathrm{WT}}-0.11 \pm$ 0.015; OGD+ eGFP-gephyrin $268 \mathrm{~A} / \mathrm{S} 270 \mathrm{~A}-0.44 \pm 0.12$. (i) Quantification of gephyrin cluster volume $(* * p<0.01$, Two-way ANOVA with Bonferroni multiple comparison test). Mean volume: Control + eGFP-gephyrin $_{\mathrm{WT}}-0.85 \pm 0.012$; Control + eGFP-gephyrin ${ }_{\mathrm{S} 268 \mathrm{~A} / \mathrm{S} 270 \mathrm{~A}}-0.22 \pm 0.043 ; \mathrm{OGD}+$ eGFP-gephyrin ${ }_{W T}-0.046 \pm 0.01$; OGD + eGFP-gephyrin ${ }_{\mathrm{S} 268 \mathrm{~A} / \mathrm{S} 270 \mathrm{~A}}-0.21 \pm 0.049$. Data shown as mean \pm s.d.

Figure 5 - Synapse changes in the peri-infarct area of ipsi-lateral hemispheres 24 h following MCAO.

(a) Example composite images for glutamatergic synaptic proteins (VGLUT1 and PSD95) and GABAergic synaptic markers (GAD65/67, $\gamma 2$ and $\alpha 5 \mathrm{GABA}_{\mathrm{A}} \mathrm{Rs}$ ). (b) Quantification for VGLUT1 cluster density (One-way ANOVA, Bonferroni multiple comparison post-hoc test; $F(2,49)=11.2$; 
$\mathrm{P}<0.0001)$. (c) Quantification for PSD95 cluster density (One-way ANOVA, Bonferroni multiple comparison post-hoc test; $\mathrm{F}(2,49)=0.84 ; \mathrm{P}=0.43$ ). (d-f) Quantification for GAD65/67 (One-way ANOVA, Bonferroni multiple comparison post-hoc test; $\mathrm{F}(2,10)=7.9 ; \mathrm{P}=0.0085$ ), $\gamma 2$ (One-way ANOVA, Bonferroni multiple comparison post-hoc test; $\mathrm{F}(2,9)=6.4 ; \mathrm{P}=0.018)$ and $\alpha 5 \mathrm{GABA}_{\mathrm{A}} \mathrm{R}$ cluster density (One-way ANOVA, Bonferroni multiple comparison test; $\mathrm{F}(2,9)=8.3 ; \mathrm{P}=0.0088$ ). Data shown as mean \pm s.d. ( $\mathrm{n}=5$ animals); $* \mathrm{P}<0.05 ; * * \mathrm{P}<0.01 ; * * * \mathrm{P}<0.001$. (g-i) WB analysis for phospho-ERK1/2, total ERK1/2, GSK3 $\beta$ and actin in sham and $24 \mathrm{~h}$ post MCAO samples. (j-j'”,) WB analysis for total gephyrin, phospho gephyrin-S268 and phospho gephyrin-S270 levels in BL6 WT sham and $24 \mathrm{~h}$ post MCAO mice. Data shown as mean \pm s.d. ( $\mathrm{n}=4$ animals).

Figure 6 - Synapse loss is attenuated in GphnS268A/S270A mutant mice 24 h following MCAO.

(a) Example images of immunohistochemical stainings from motor cortex L2/3 ipsi- and contralateral sides in GphnS268A/S270A mutant mice $24 \mathrm{~h}$ following MCAO. Glutamatergic synaptic terminals were visualised using VGLUT1 and PSD95 (see inset). GABAergic synaptic sites were visualised using GAD65/67, $\gamma 2$ and $\alpha 5 \mathrm{GABA}_{\mathrm{A}}$ Rs. (b) Quantification for VGLUT1 (One-way ANOVA, Brown-Forsythe test; $\mathrm{F}(2,11)=0.35 ; \mathrm{P}=0.70$ ). (b') Quantification of PSD95 cluster density (One-way ANOVA, Brown-Forsythe test; $\mathrm{F}(2,11)=1.03 ; \mathrm{P}=0.38$ ). (c) Quantification for GAD65/67 (One-way ANOVA, Bonferroni multiple comparison test; $\mathrm{F}(2,9)=11.9 ; \mathrm{P}=0.003$ ). (c') Quantification of $\gamma 2$ (One-way ANOVA, Bonferroni multiple comparison test; $F(2,11)=0.60$; $\mathrm{P}=0.56$ ). (c') Quantification of $\alpha 5 \mathrm{GABA}_{\mathrm{A}}$ Rs clusters (One-way ANOVA, Bonferroni multiple comparison test; $\mathrm{F}(2,11)=4.9 ; \mathrm{P}=0.02)$. Data shown as mean \pm s.d. ( $\mathrm{n}=5$ animals). 
Figure 7 - GphnS268A/S270A mutant mice show reduced neuroinflammation 24 h following

MCAO. (a) Quantification of infarct volume in BL6 WT, BL6 WT treated with PLX5622, $\mathrm{BDNF}^{\text {flox/flox }} / \mathrm{CX} 3 \mathrm{CR} 1^{\mathrm{ERT} 2 \mathrm{Cre}+/-}$ and GphnS268A/S270A mutant mice at $24 \mathrm{~h}$ following MCAO (One-way ANOVA, Brown-Forsythe test; $\mathrm{F}(3,15)=8.9 ; \mathrm{P}=0.0028$ ). (b) Example image of volumetry analysis from $24 \mathrm{~h}$ post MCAO tissue sections from BL6 WT, BL6 WT treated with PLX5622, BDNF ${ }^{\text {flox/flox }} / \mathrm{CX} 3 \mathrm{CR} 1^{\mathrm{ERT2Cre+/-}}$ and GphnS268A/S270A mutant mice. Results expressed as mean \pm s.d. ( $\mathrm{N}=5$ or more). (c) Example images of BL6 WT mice stained from sham or $24 \mathrm{~h}$ post MCAO tissue for microglia markers IBA1 and CD11b (IBA1 shown). (c') Quantification for microglia density in sham animals and ipsi- and contra-lateral sides of MCAO animals. (c') Quantification microglia area covered using IBAI staining from sham animals, ipsiand contra-lateral sides of MCAO animals. (c"') Quantification for CD11b intensity in sham animals, ipsi- and contra-lateral sides after MCAO animals. (d) Example images of GphnS268A/S270A mutant mice stained from sham or $24 \mathrm{~h}$ post MCAO tissue for microglia markers IBA1 and CD11b (IBA1 shown). (d') Quantification for microglia density in sham animals and ipsi- and contra-lateral sides of MCAO animals. (d') Quantification microglia area covered using IBAI staining from sham animals, ipsi- and contra-lateral sides of MCAO animals. (d"') Quantification for CD11b intensity in sham animals, ipsi- and contra-lateral sides after MCAO animals. Results expressed as mean \pm s.d. ( $\mathrm{n}=5$ animals); $* \mathrm{P}<0.05$ (One-way ANOVA, Bonferroni multiple comparison test).

Figure 8 - GphnS268A/S270A mutation affects BDNF levels in microglia after MCAO. (a) Example images from $\mathrm{BDNF}^{\mathrm{wt} / \mathrm{wt}} / \mathrm{CX} 3 \mathrm{CR} 1^{\mathrm{ERT} 2 \mathrm{Cre}+/-}$ mice stained from sham or $24 \mathrm{~h}$ post $\mathrm{MCAO}$ tissue for microglia markers IBA1 and BDNF (composite shown). (a') Quantification for BDNF 
intensity within IBA1 positive cells in $\mathrm{BDNF}^{\mathrm{wt} / \mathrm{wt}} / \mathrm{CX} 3 \mathrm{CR} 1^{\mathrm{ERT} 2 \mathrm{Cre}+/-}$ sham animals and ipsi- and contra-lateral sides of MCAO animals. (b) Example images from BDNF flox/flox / CX3CR1 ${ }^{\text {ERT2Cre+/- }}$ mice stained from sham or $24 \mathrm{~h}$ post MCAO tissue for microglia markers IBA1 and BDNF. (b’) Quantification for BDNF intensity within IBA1 positive cells in BDNF flox/flox / CX3CR1 $1^{\text {ERT2Cre+/- }}$ sham animals and ipsi- and contra-lateral sides of MCAO animals. (c) Example images from GphnS268A/S270A mutant sham animals and ipsi- and contra-lateral sides of MCAO animals. (c') Quantification for BDNF intensity within IBA1 positive cells in GphnS268A/S270A mutant sham animals and ipsi- and contra-lateral sides of MCAO animals. (d) Overview of pathway(s) regulating synaptic downregulation downstream of microglia release proBDNF and mBDNF; (1) an unknown signal from GABAergic postsynaptic sites facilitates microglia hyper reactivity after MCAO; (2) activated microglia produce and release proBDNF and mBDNF to act on their cognate receptors $\mathrm{p} 75^{\mathrm{NTR}}$ and TrkB respectively; (3) proBDNF via $\mathrm{p} 75^{\mathrm{NTR}}$ activates RhoA/Rac1 pathway for actin remodeling and spine loss, triggering microglia aided stripping of VGLUT terminals; (4) ERK1/2 and GSK3 $\beta$ pathways are activated downstream of TrkB receptors to phosphorylate gephyrin at S268 and S270 residues respectively; (5) gephyrin phosphorylation at S268 and S270 leads to gephyrin scaffold loss via calpain-1 clevage to facilitate $G_{A B A} R$ internalization and microglia aided displacement of GABAergic terminals. This mechanism outlines how excitatory and inhibitory synapses are lost at both ipsi- and contra-lateral sides $24 \mathrm{~h}$ post ischemia. 
bioRxiv preprint doi: https://doi.org/10.1101/2020.04 22 055087: this version posted December 20, 2020. The copyright holder for this preprint (which was not certified by peer review) is the author/funder, who has granted bioRxiv a license to display the preprint in perpetuity. It is made available under aCC-BY-ND 4.0 International license.

Table 1

\begin{tabular}{|c|c|c|c|c|}
\hline Target & Host species & Dilution & Catalog \# & Company/origin \\
\hline \multicolumn{5}{|c|}{ Antibodies used for immunohistochemistry } \\
\hline GABAAR $\gamma 2$ & Guinea pig & $1: 2000$ & - & Home-made \\
\hline GABAAR $\alpha 5$ & Guinea pig & $1: 3000$ & - & Home-made \\
\hline Gad-65/67 & Rabbit & $1: 1000$ & GC 3008 & Affiniti \\
\hline Vglut1 & Guinea pig & $1: 1000$ & 135304 & Synaptic Systems \\
\hline PSD95 & Mouse & $1: 1000$ & $73-028$ & NeuroMap \\
\hline GFAP & Mouse & $1: 2000$ & MAB360 & Millipore \\
\hline Iba-1 & Rabbit & $1: 1000$ & $019-19741$ & Wako \\
\hline Cd11b & Rat & $1: 50$ & 550282 & BD-Pharmingen \\
\hline mBDNF N-9 & Mouse & $1: 100$ & BDNF \#9 & DSHB \\
\hline $\mathrm{p} 75^{\mathrm{NTR}}$ & Rabbit & $1: 200$ & AB-NO1 & $\begin{array}{l}\text { Advanced Targeting } \\
\text { System }\end{array}$ \\
\hline pro-BDNF \#B240 & Mouse & $1: 200$ & & Philip Barker \\
\hline \multicolumn{5}{|c|}{ Antibodies used for Western Blot } \\
\hline GABAAR $\alpha 1$ & Rabbit & $1: 600$ & - & Home-made \\
\hline GABAAR $\alpha 2$ & Rabbit & $1: 1000$ & - & Home-made \\
\hline Gephyrin & Mouse & $1: 1000$ & 147111 & Synaptic Systems \\
\hline Gephyrin PS268 & Rabbit & $1: 500$ & - & Home-made \\
\hline Gephyrin PS270 & Rabbit & $1: 500$ & - & Home-made \\
\hline GSK3 $\beta$ & Rabbit & $1: 1000$ & ab124661 & Abcam \\
\hline Erk1/2 & Rabbit & $1: 4000$ & $9102 \mathrm{~S}$ & Cell Signaling \\
\hline $\begin{array}{l}\text { Phospho-Erk1/2 } \\
\text { (Thr202/Tyr204) }\end{array}$ & Rabbit & $1: 4000$ & 9101 & Cell Signaling \\
\hline BDNF & Rabbit & $1: 3000$ & Ab108319 & Abcam \\
\hline Actin & Mouse & $1: 10,000$ & MAB1501 & Millipore \\
\hline
\end{tabular}


b

2.0

a bioRxiv preprint doi: https://doiorg/10.1101/2020.04.22:055087; thro vepstoh posted Decem
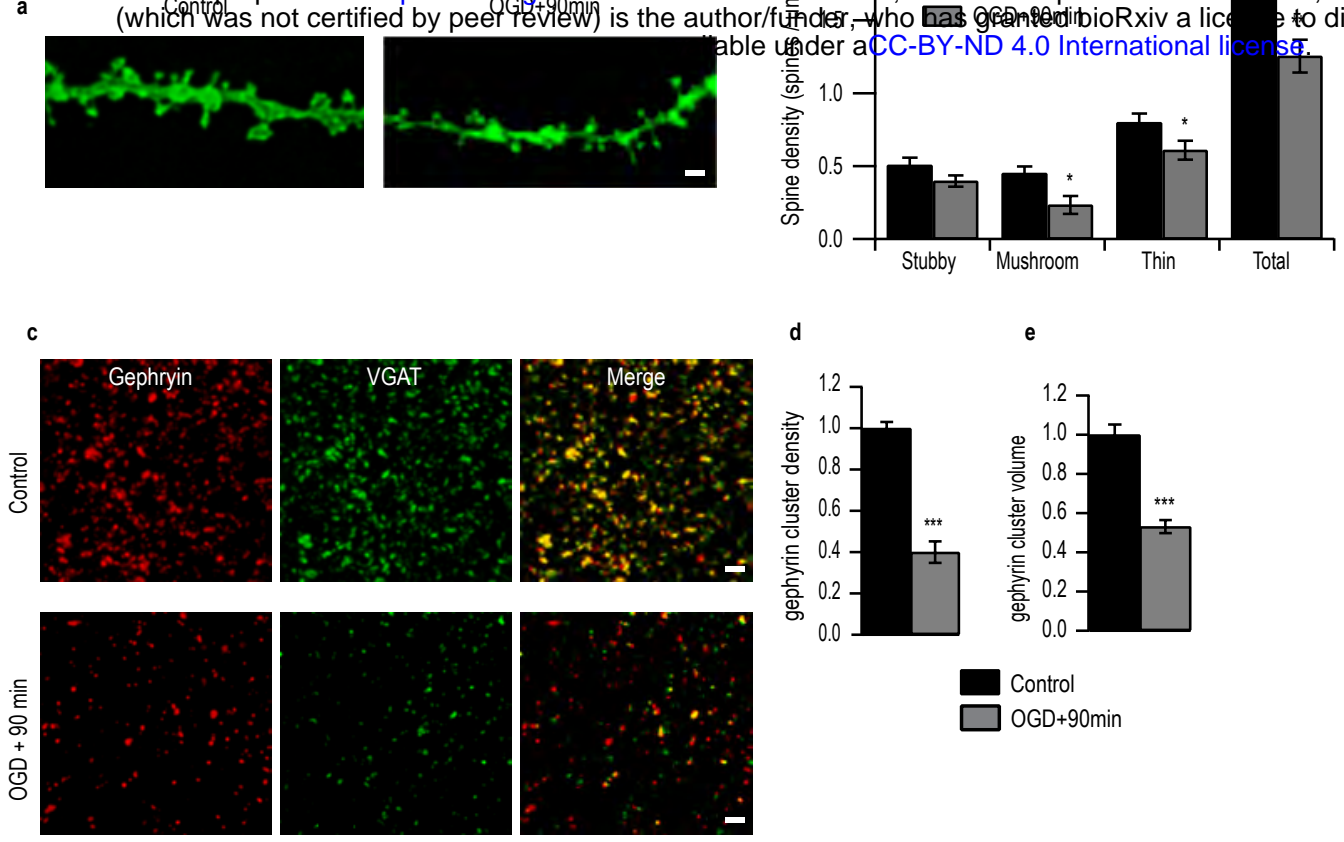

e
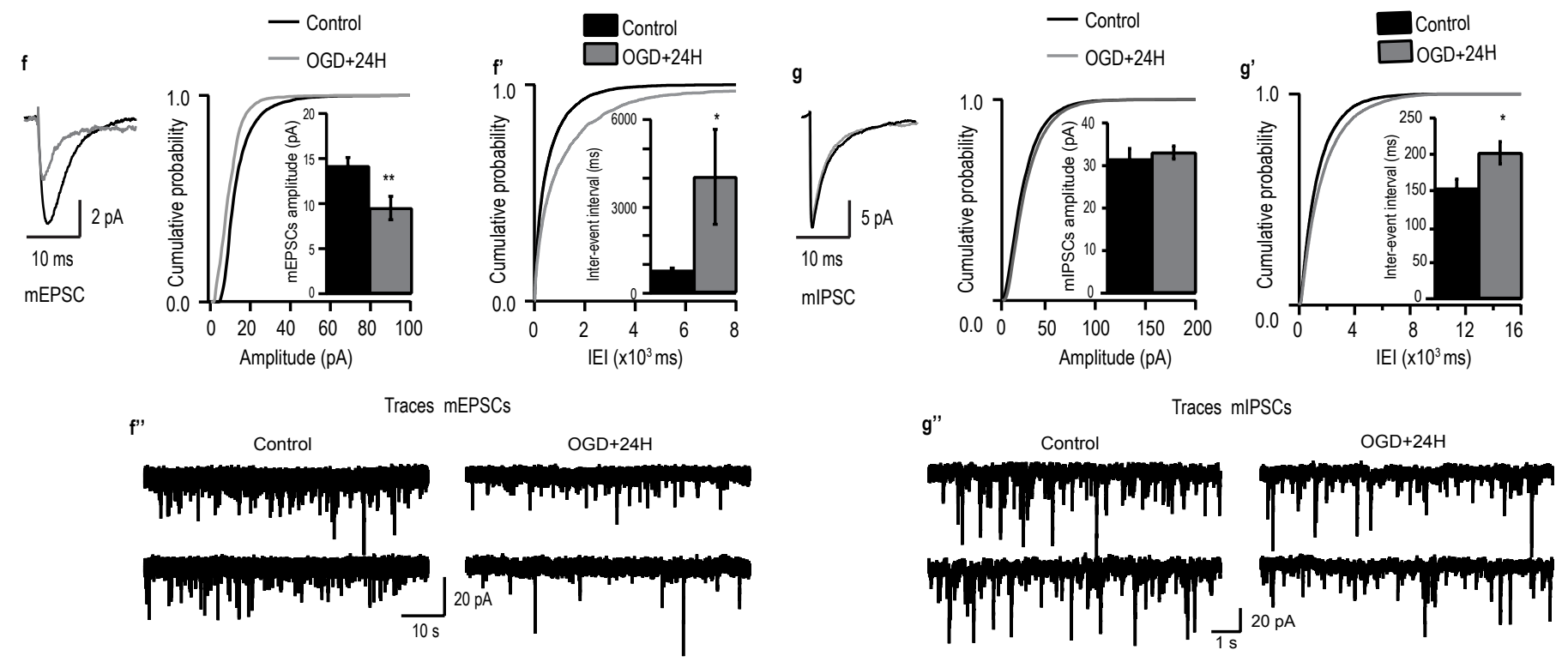

Cramer et al., Figure 1. 

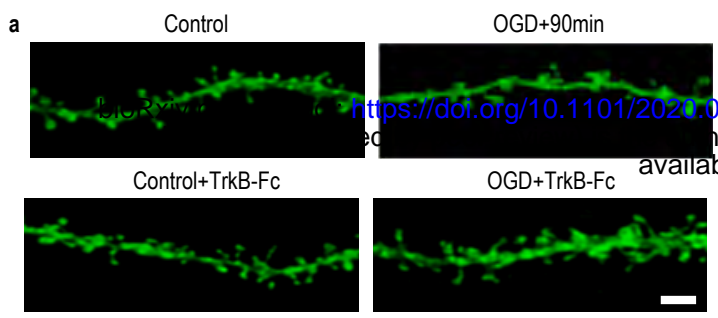

c
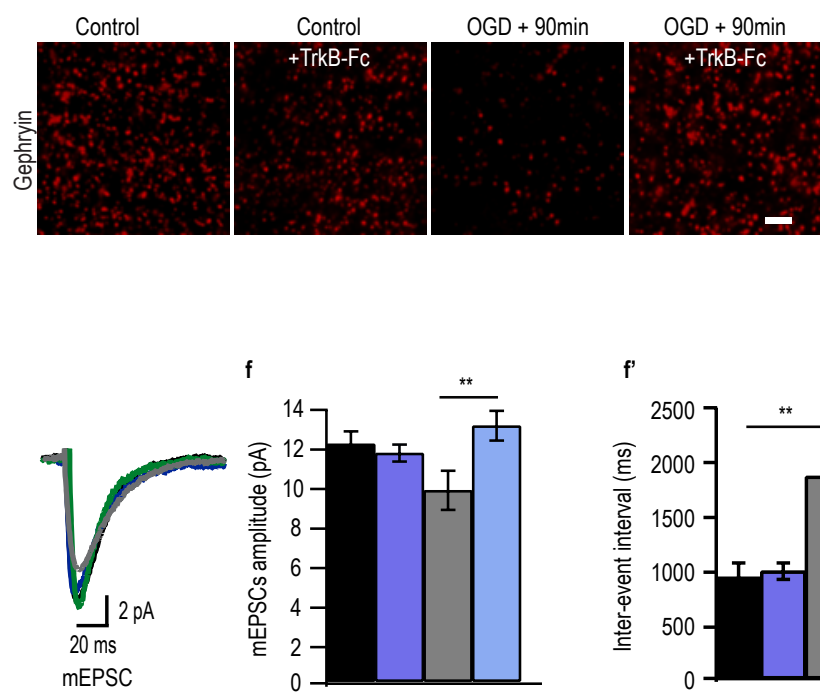

b 1.6

1.6
$1.4-\square$ Control
$\square$ Control+TrkB-Fc or/furfer, who has granted bioRxiv a license to displ the preprint in perpetuity. It is made .

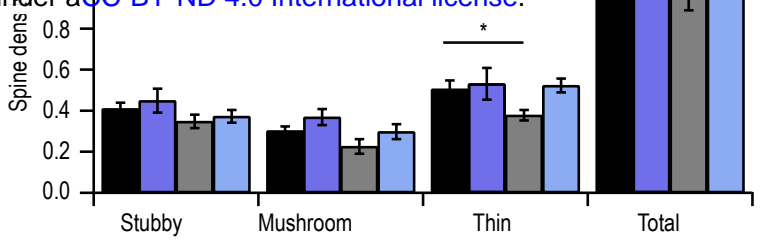

d
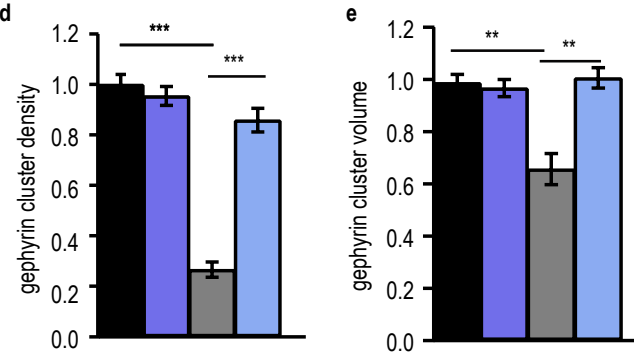

Control

$\square$ Control+TrkB-Fc

$\square$ OGD+90min

$\square$ oGD+TrkB-Fc
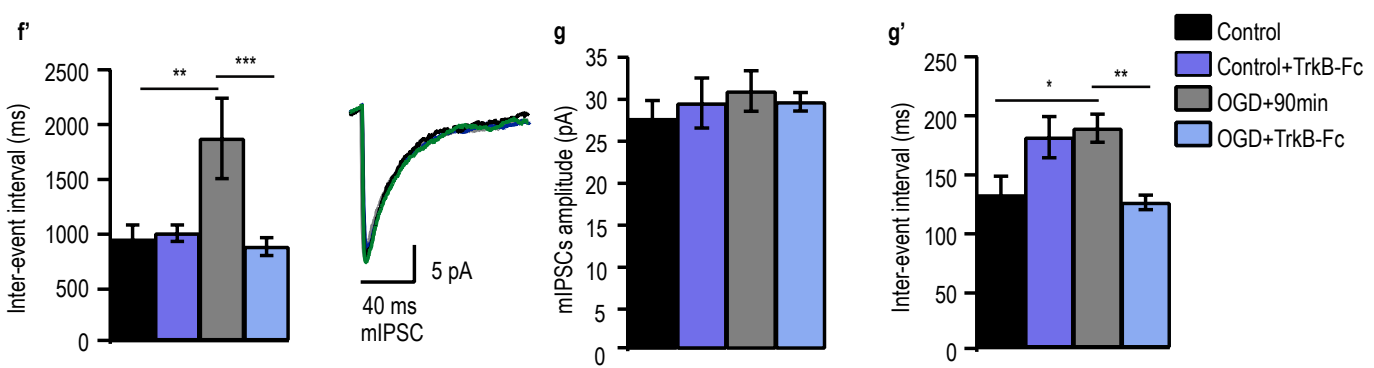

Cramer et al., Figure 2 

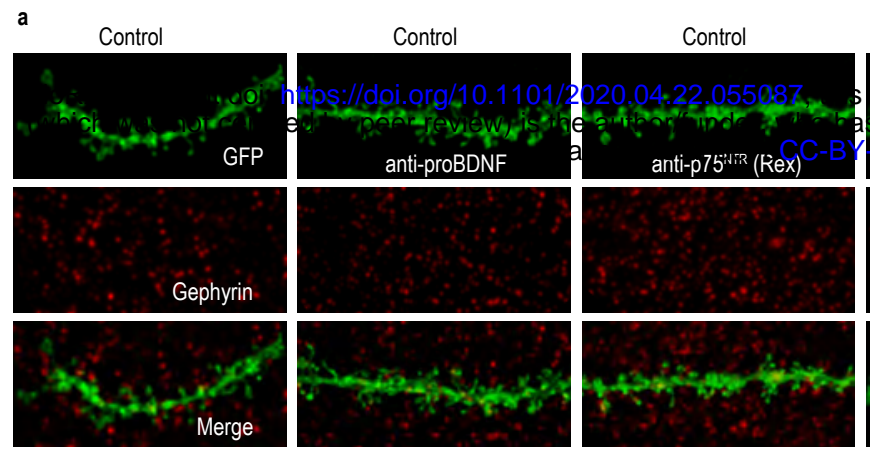

$O G D+90 \mathrm{~min}$

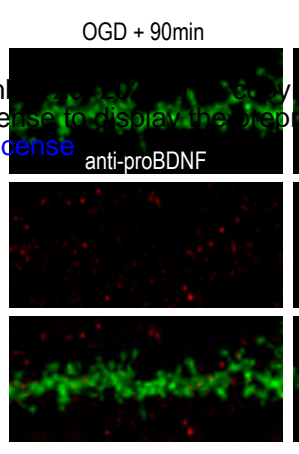

$O G D+90 \mathrm{~min}$
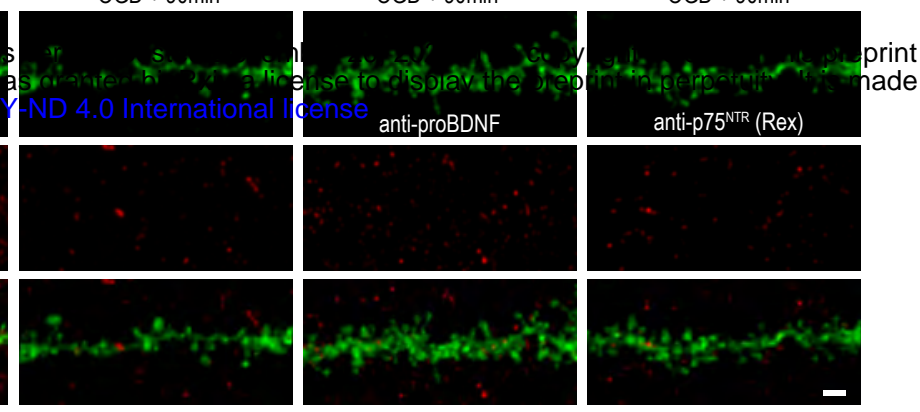

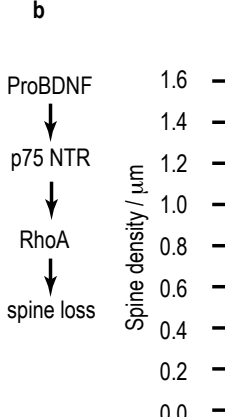

\section{Control}

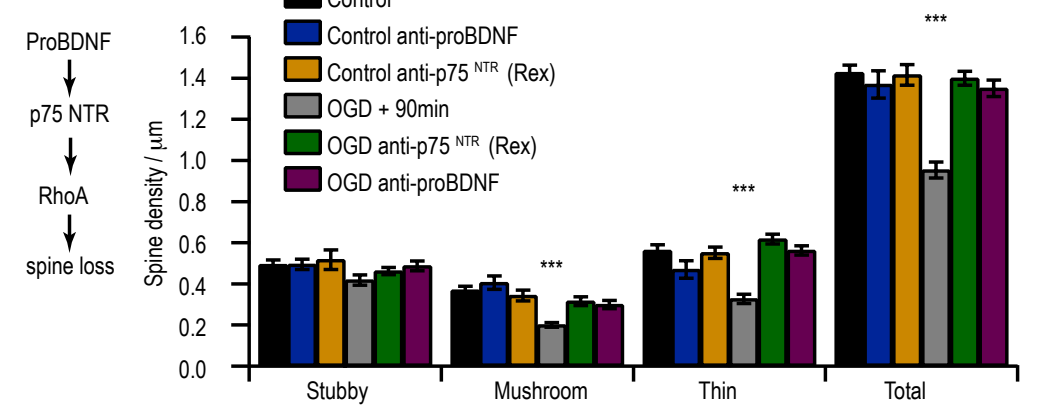

c
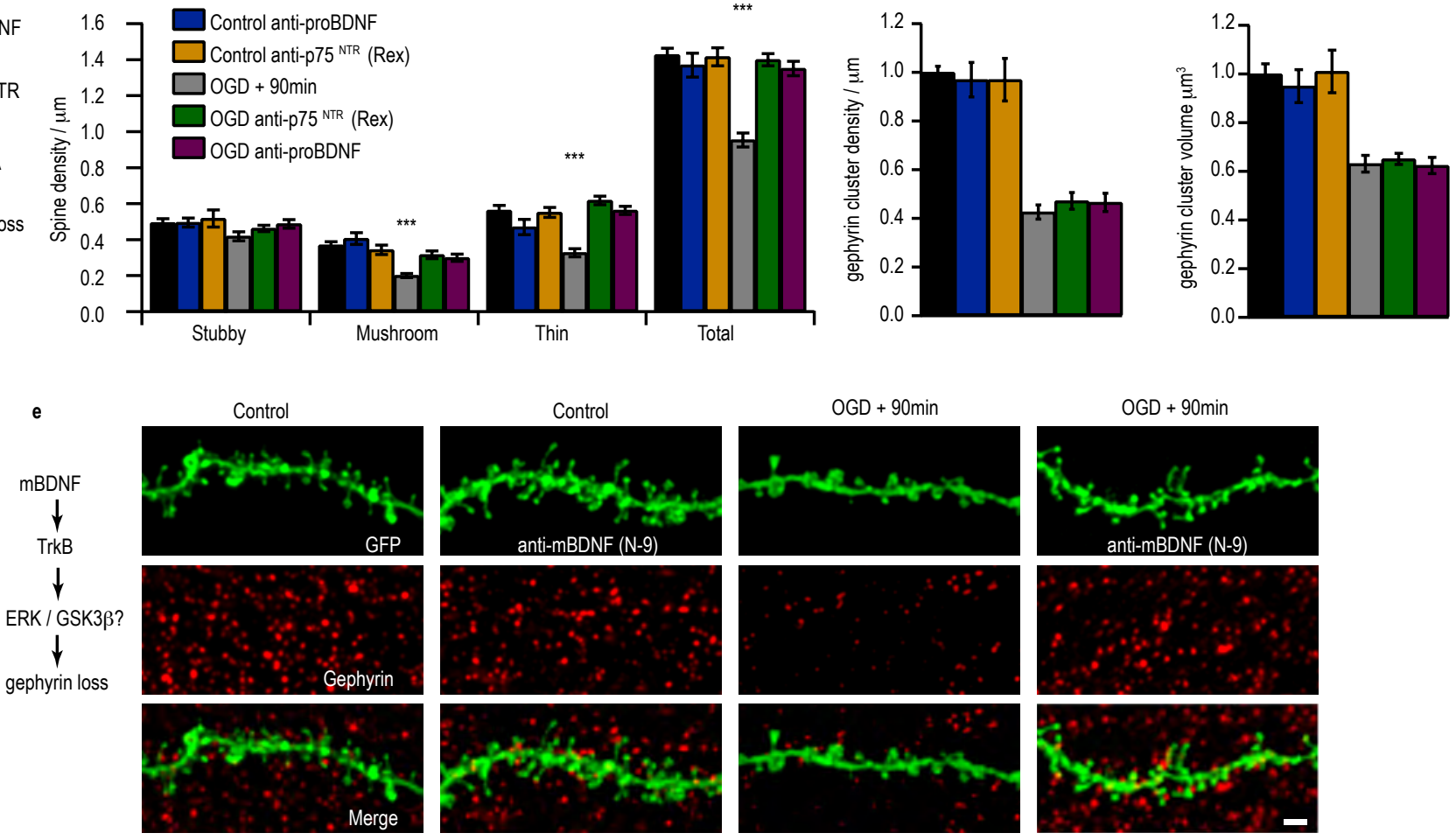

$$
\mathrm{f}
$$
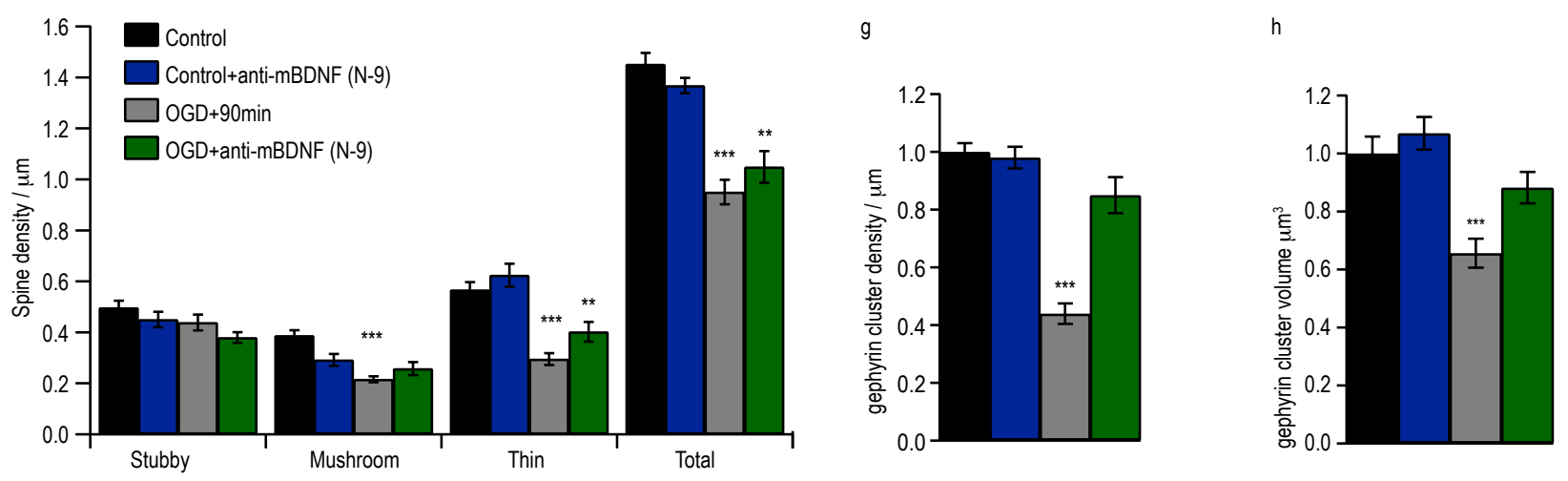

Cramer et al., Figure 3 


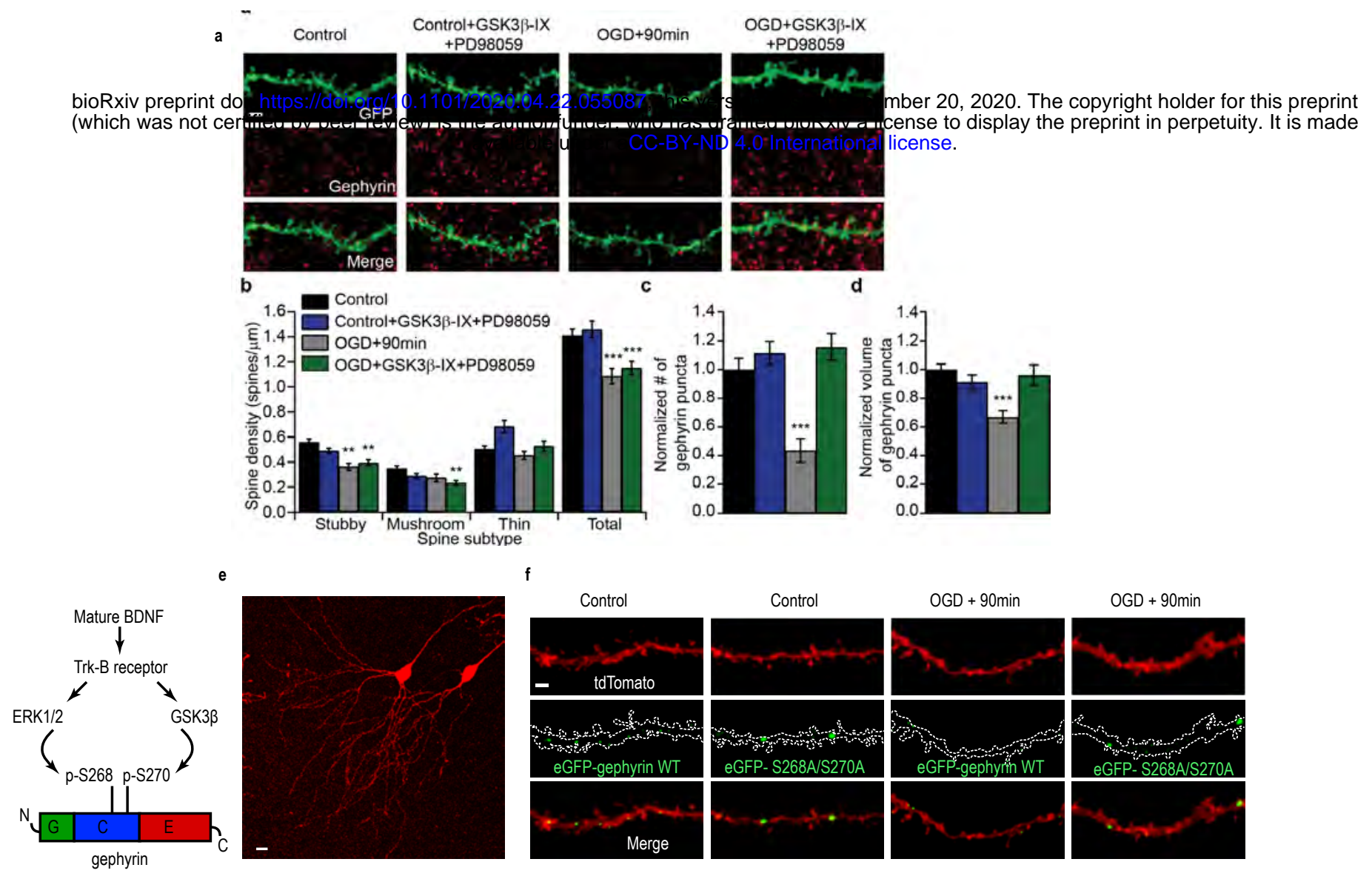

g

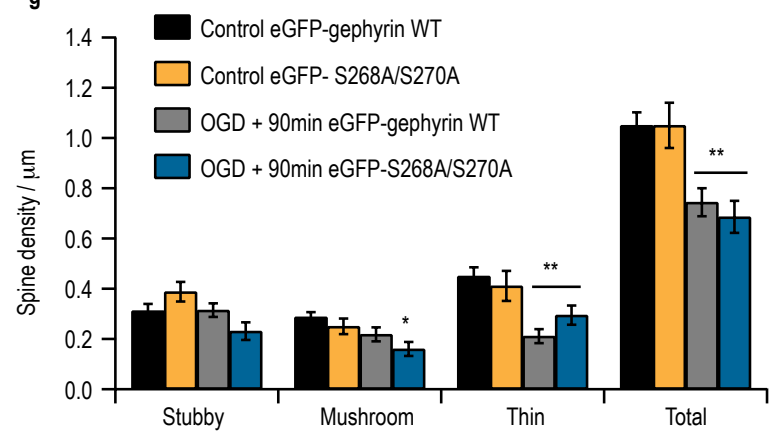

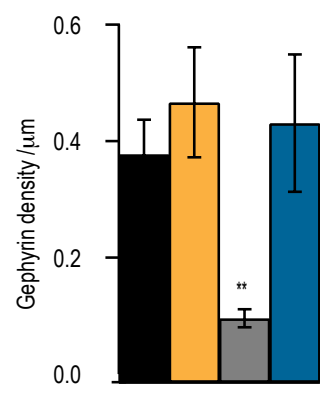

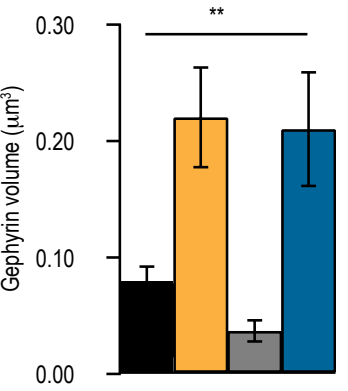

Cramer et al., Figure 4 

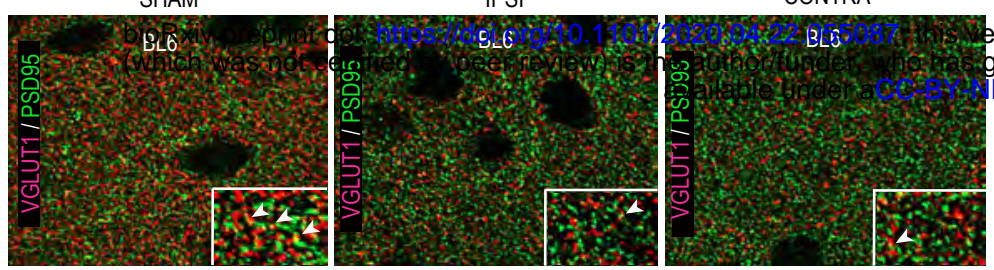

ersign 2 posted December 20, 2020. Theacongyright holder for this preprint graeted bioRxiv a license to display thempreprint in perpetuity. It is made
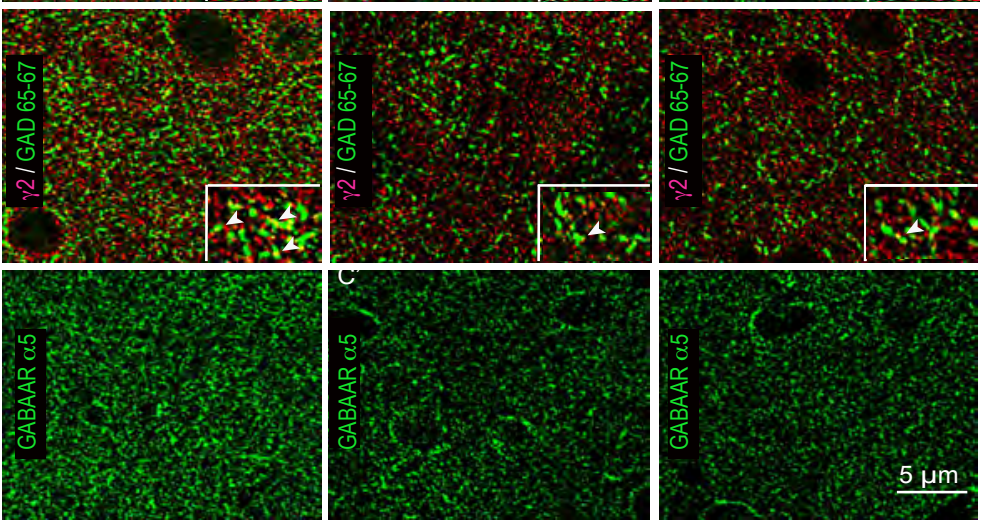
年0 internation
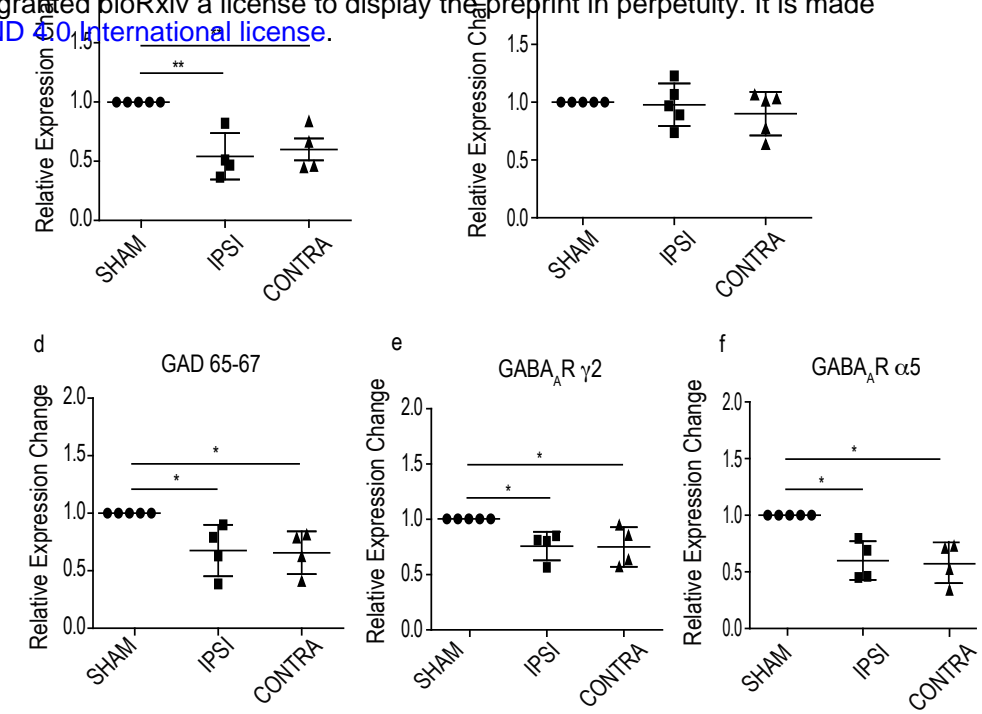

g

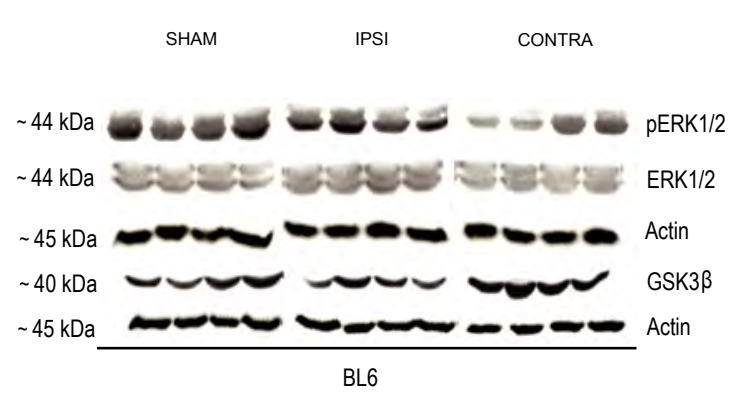

SHAM

IPSI

CONTRA

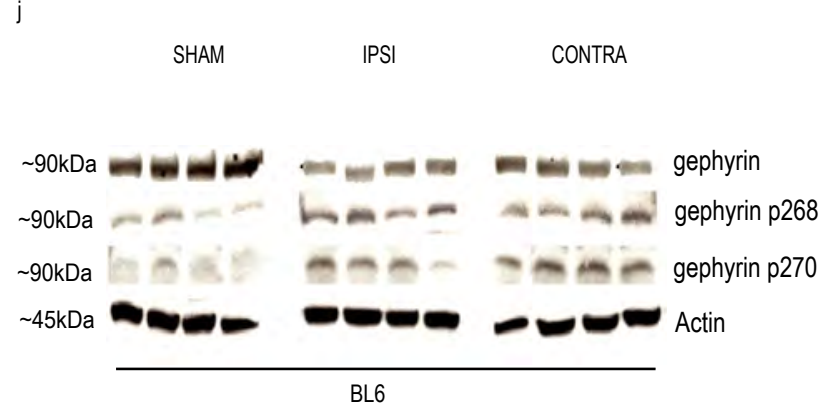

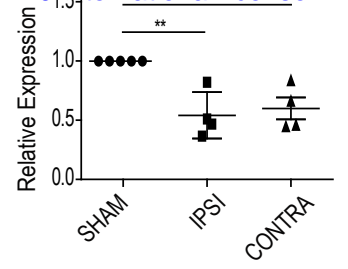
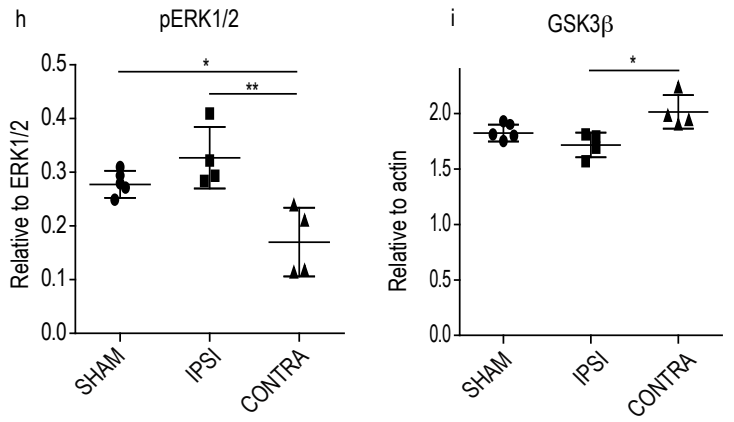

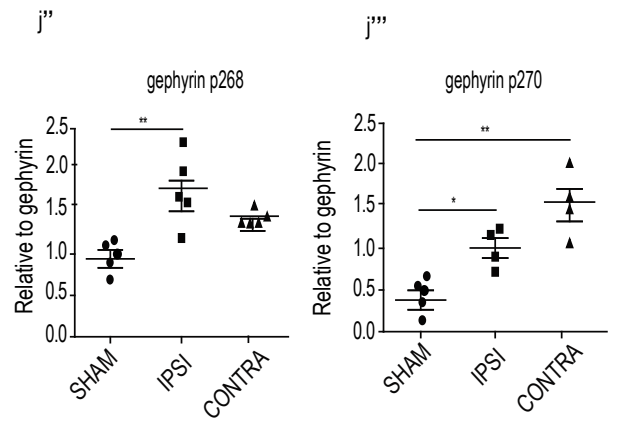

Cramer et al., Figure 5 


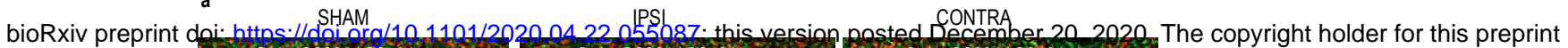
(which was not cef
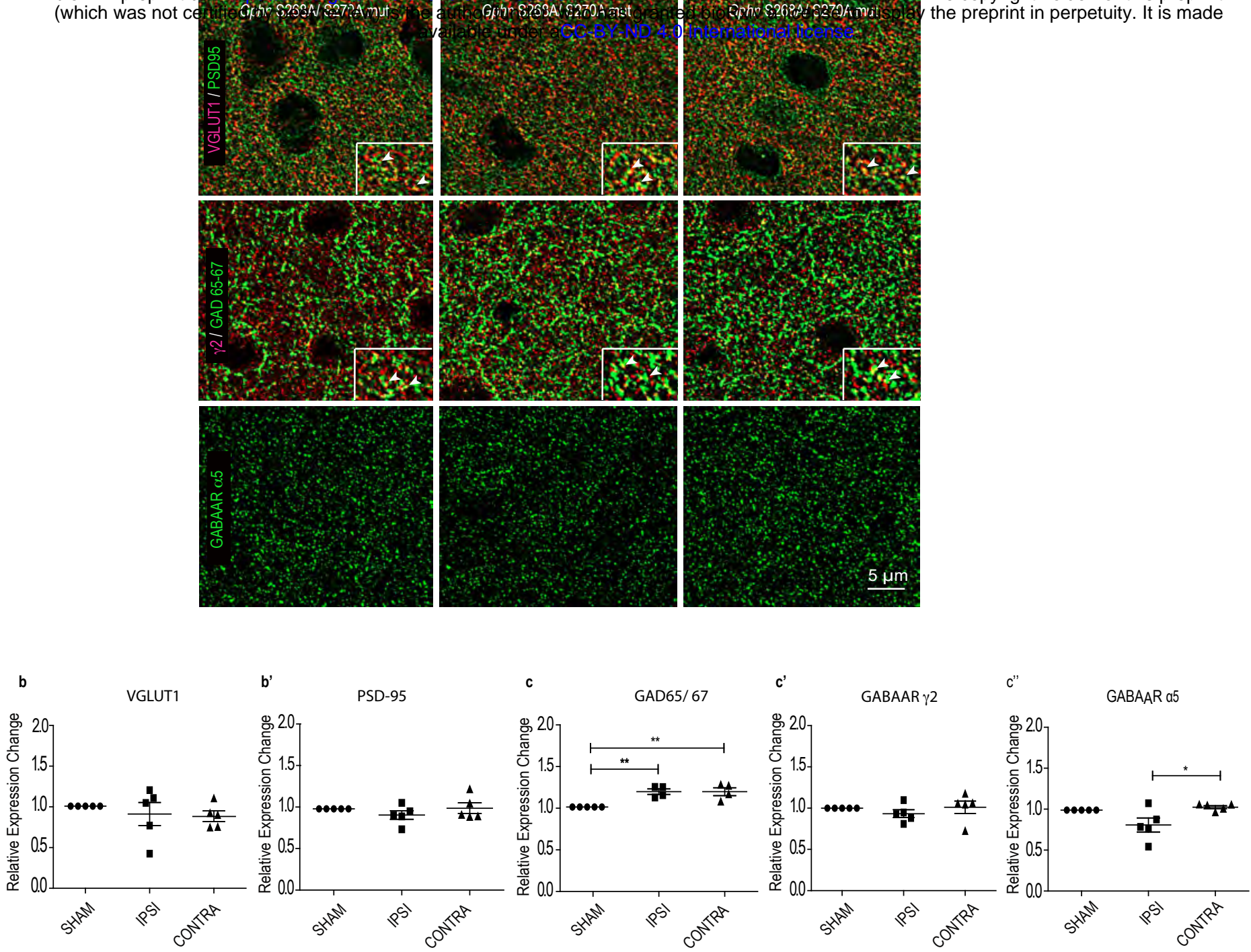

Cramer et al., Figure 6 
bioRxiv preprint doi: https://doi.org/10.1101/2020.04.22.055087; this version posted December 20, 2020. The copyright holder for this preprint (which was not certified by peer review) is the author/funder, who has granted bioRxiv a license to display the preprint in perpetuity. It is made
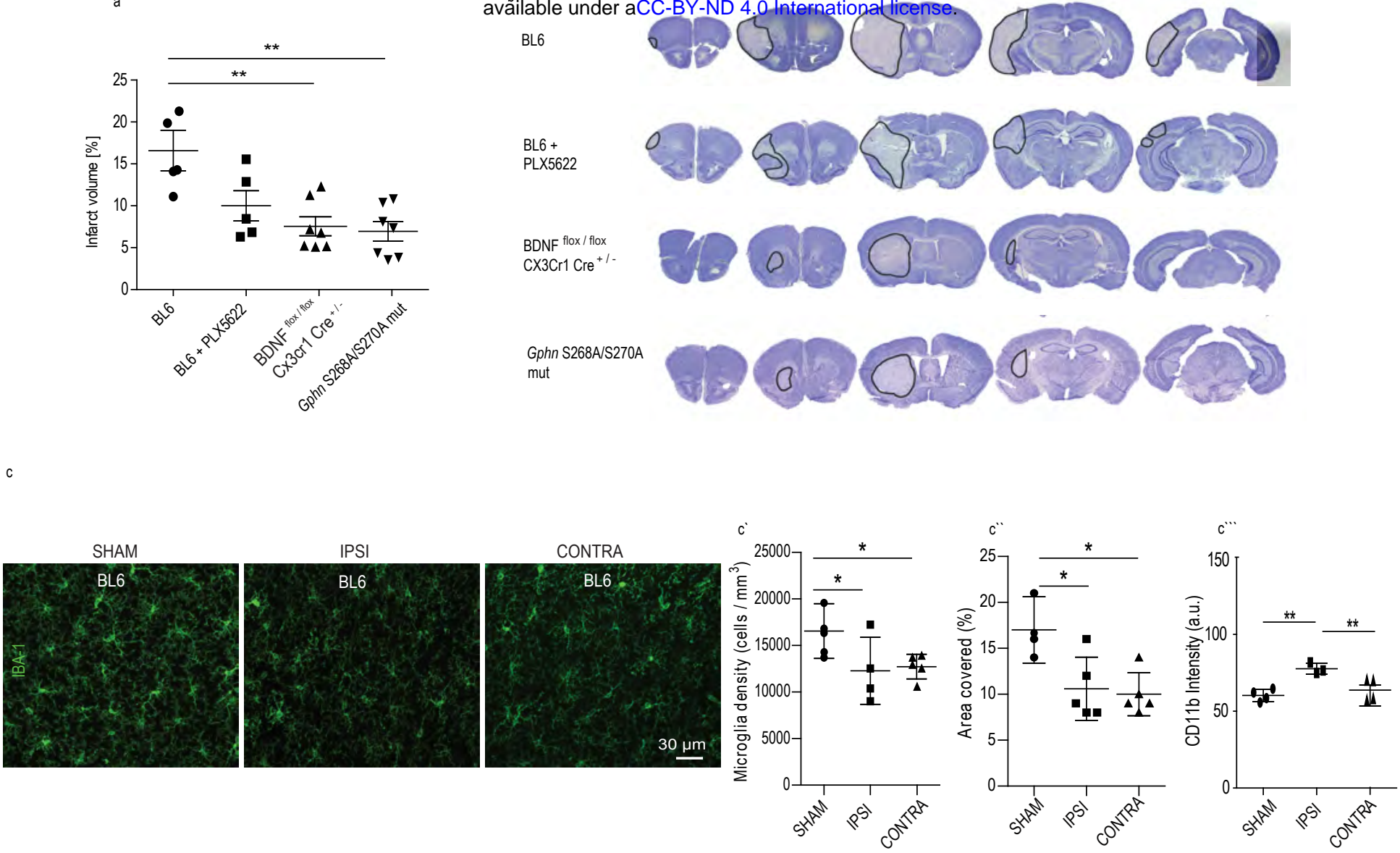

SHAM

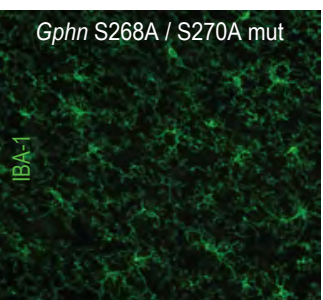

IPSI

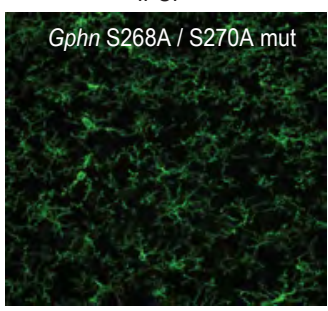

CONTRA

Gphn S268A / S270A mut
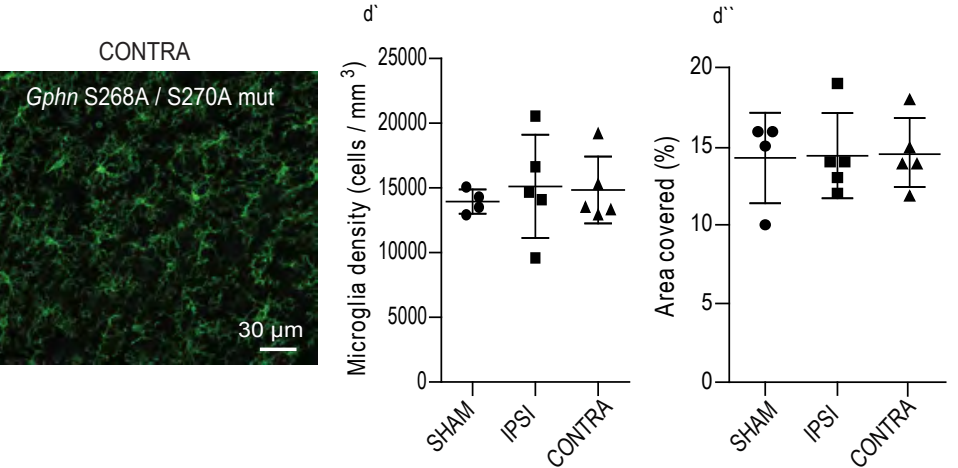

$d^{\prime \prime}$

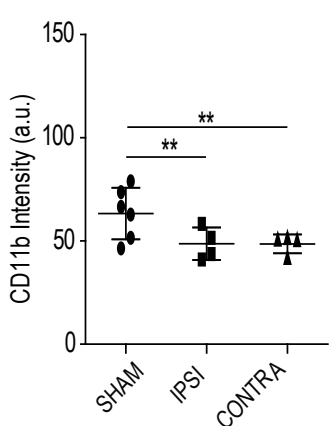




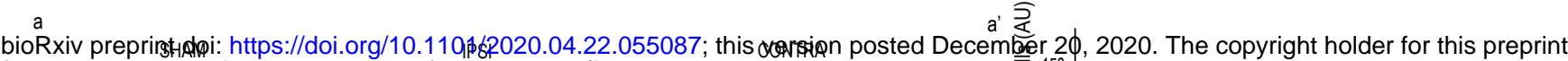
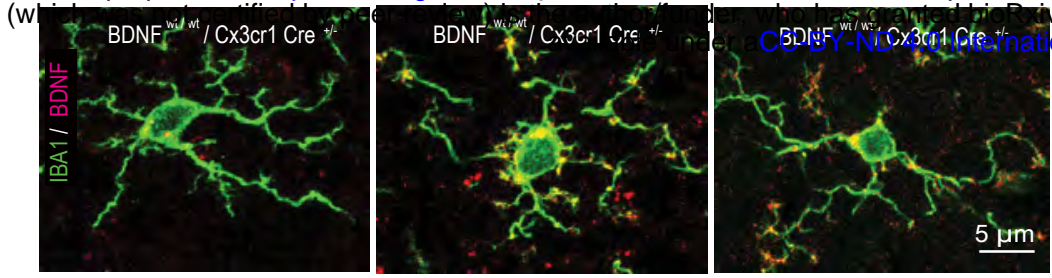

b

SHAM
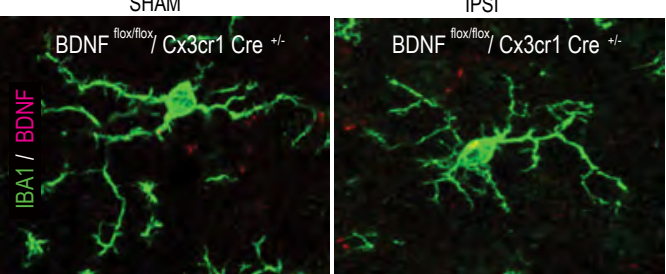

CONTRA

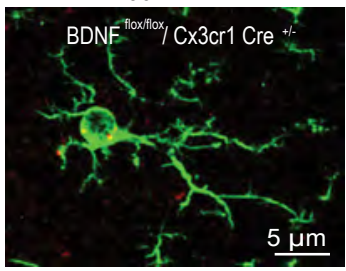

b'
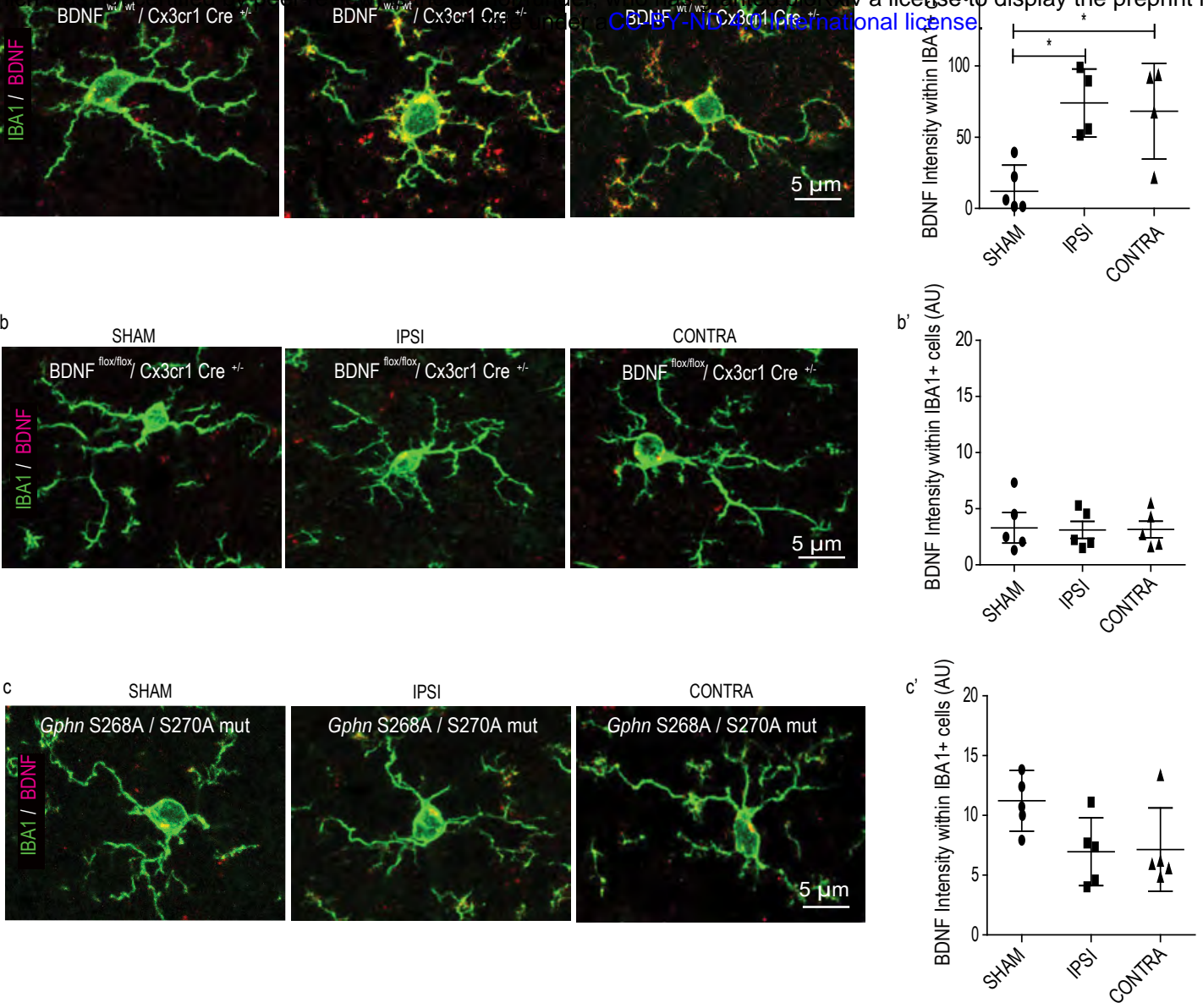

d

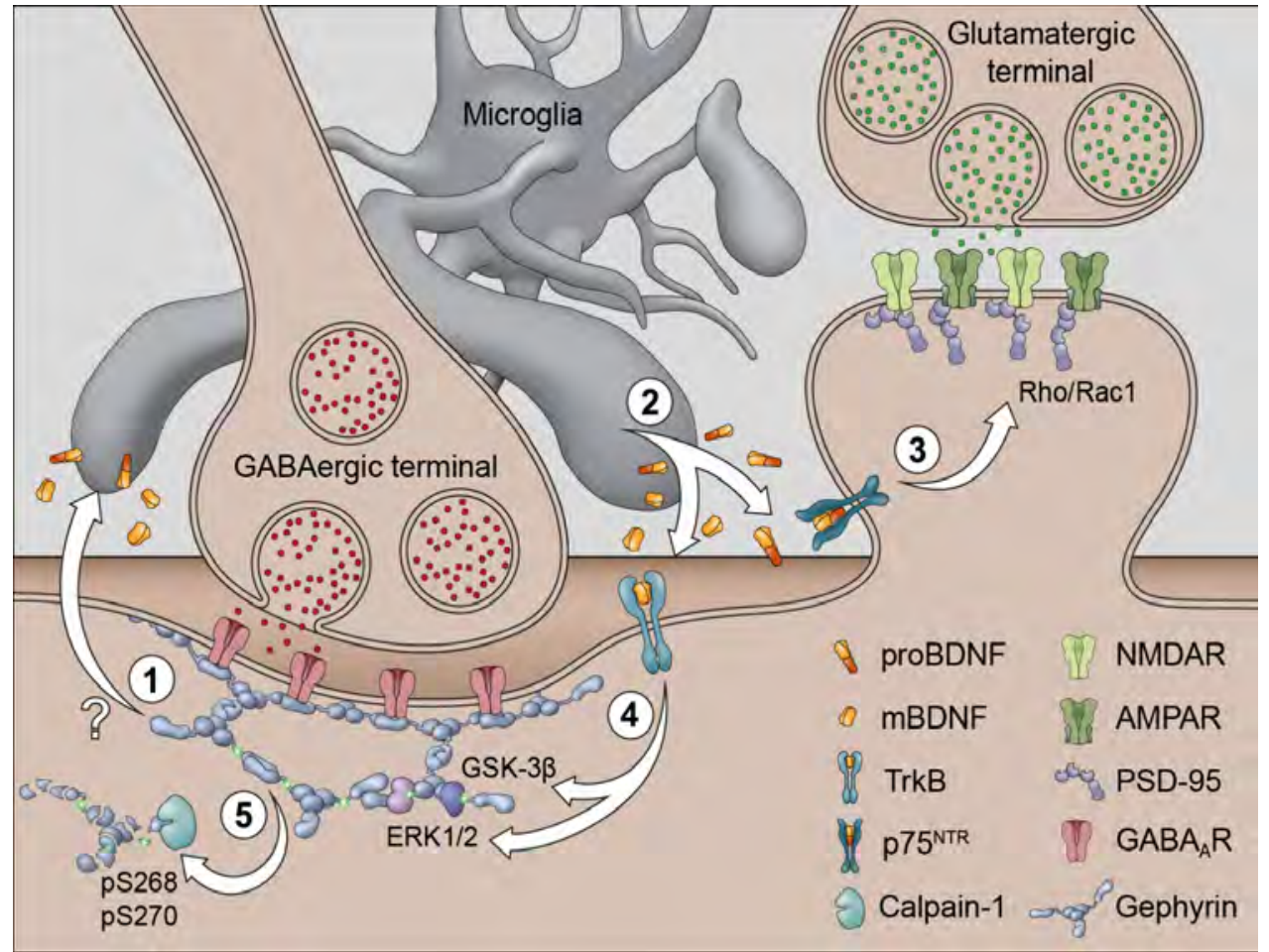

Cramer et al., Figure 8 
a

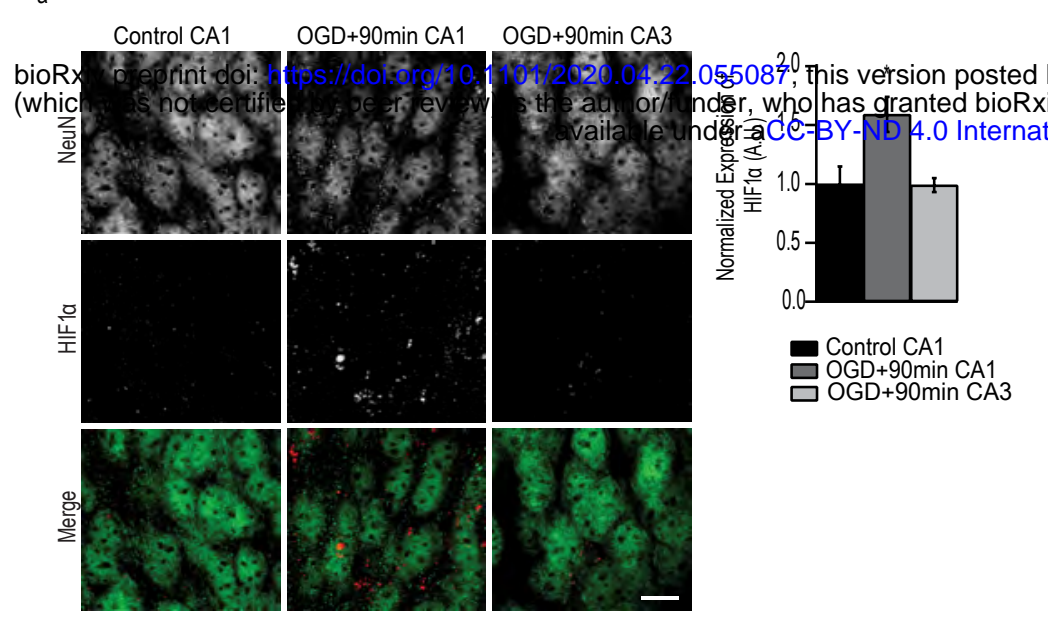

b

b'

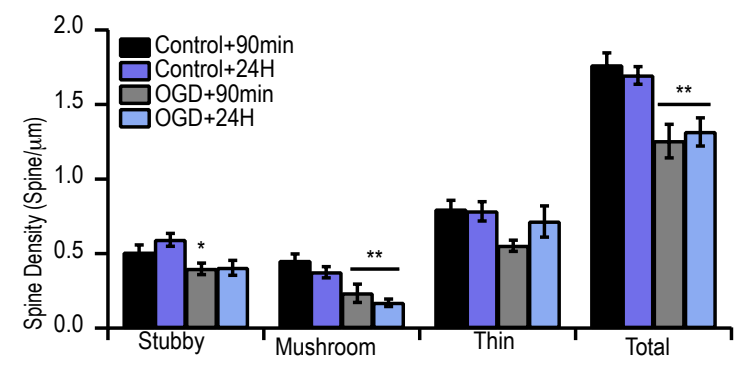

c
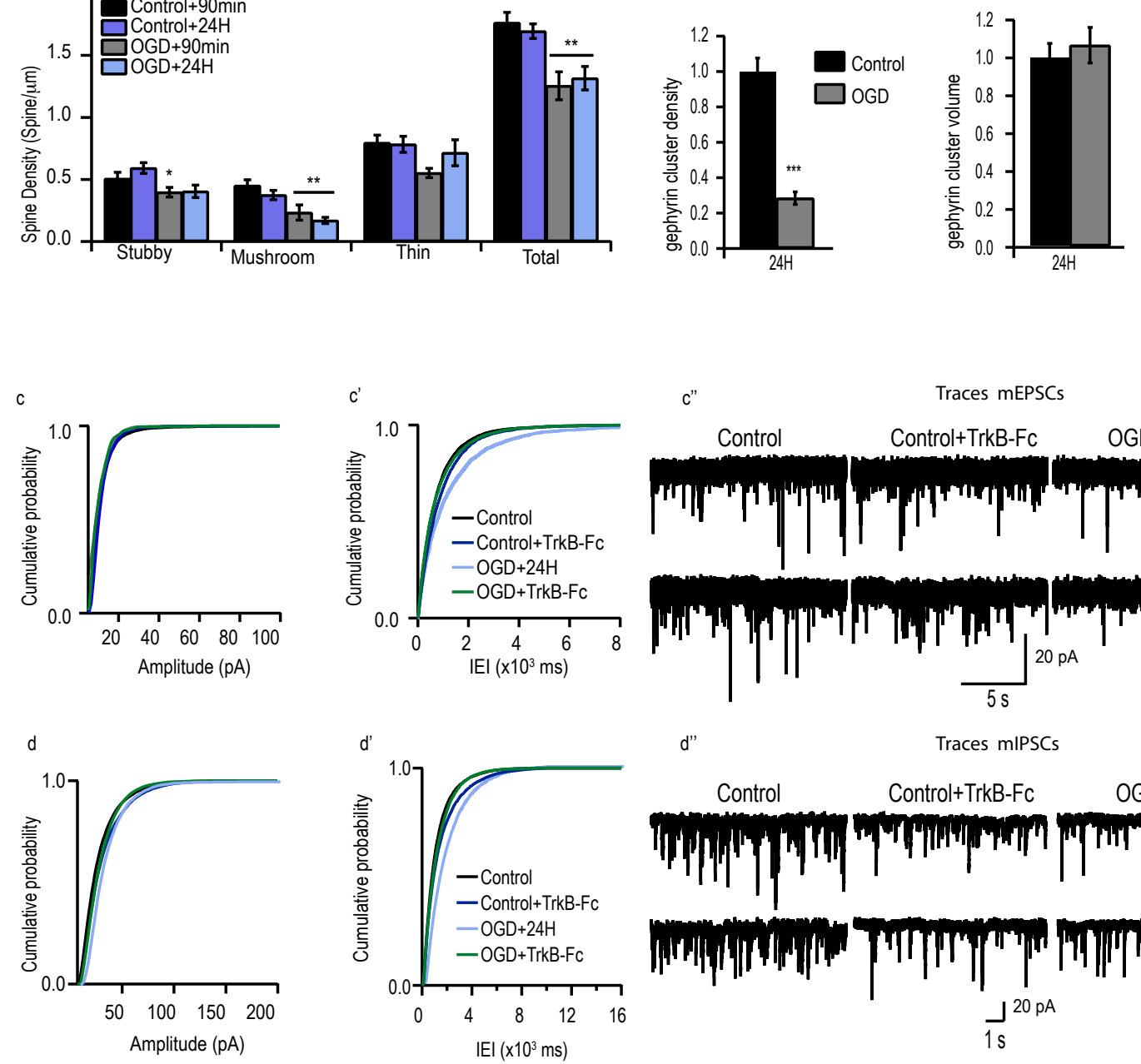

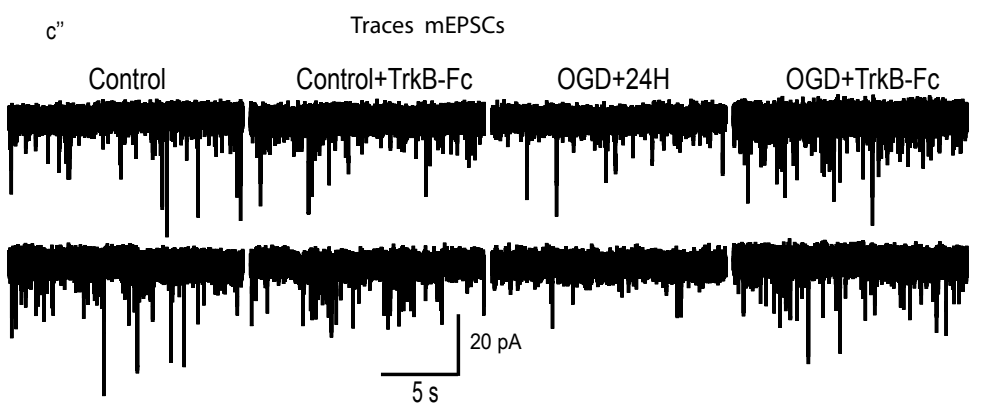

d" $\underset{\text { Control }}{\text { Control+TrkB-Fc }}$ OGD+24H

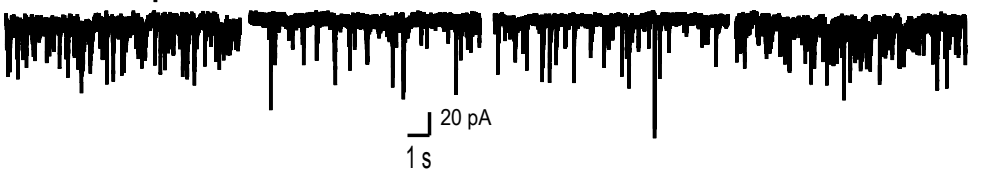


bioRxiv preprint doi: https://doi.org/10.1101/2020.04.22.055087; this version posted December 20, 2020. The copyright holder for this preprint (which was not certified by peer review) is the author/funder, who has granted bioRxiv a license to display the preprint in perpetuity. It is made available under aCC-BY-ND 4.0 International license.
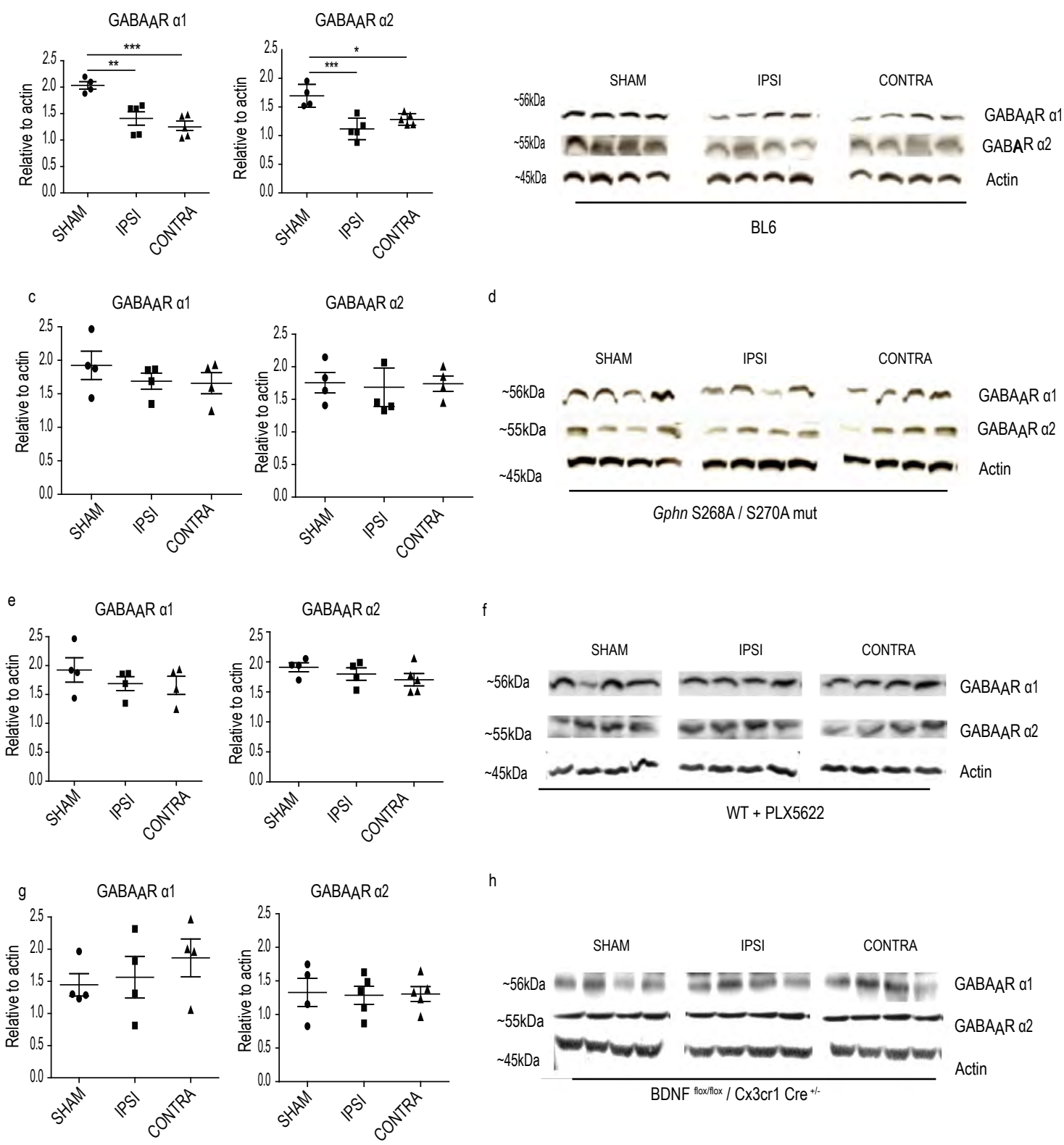

WT + PLX5622 


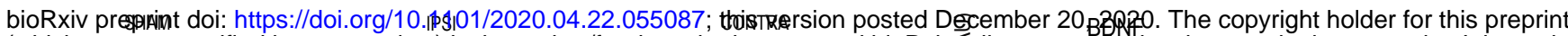
(which was not cortified by peer
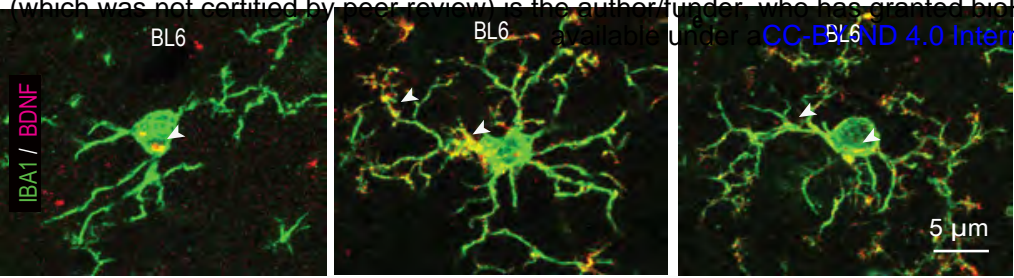

b'
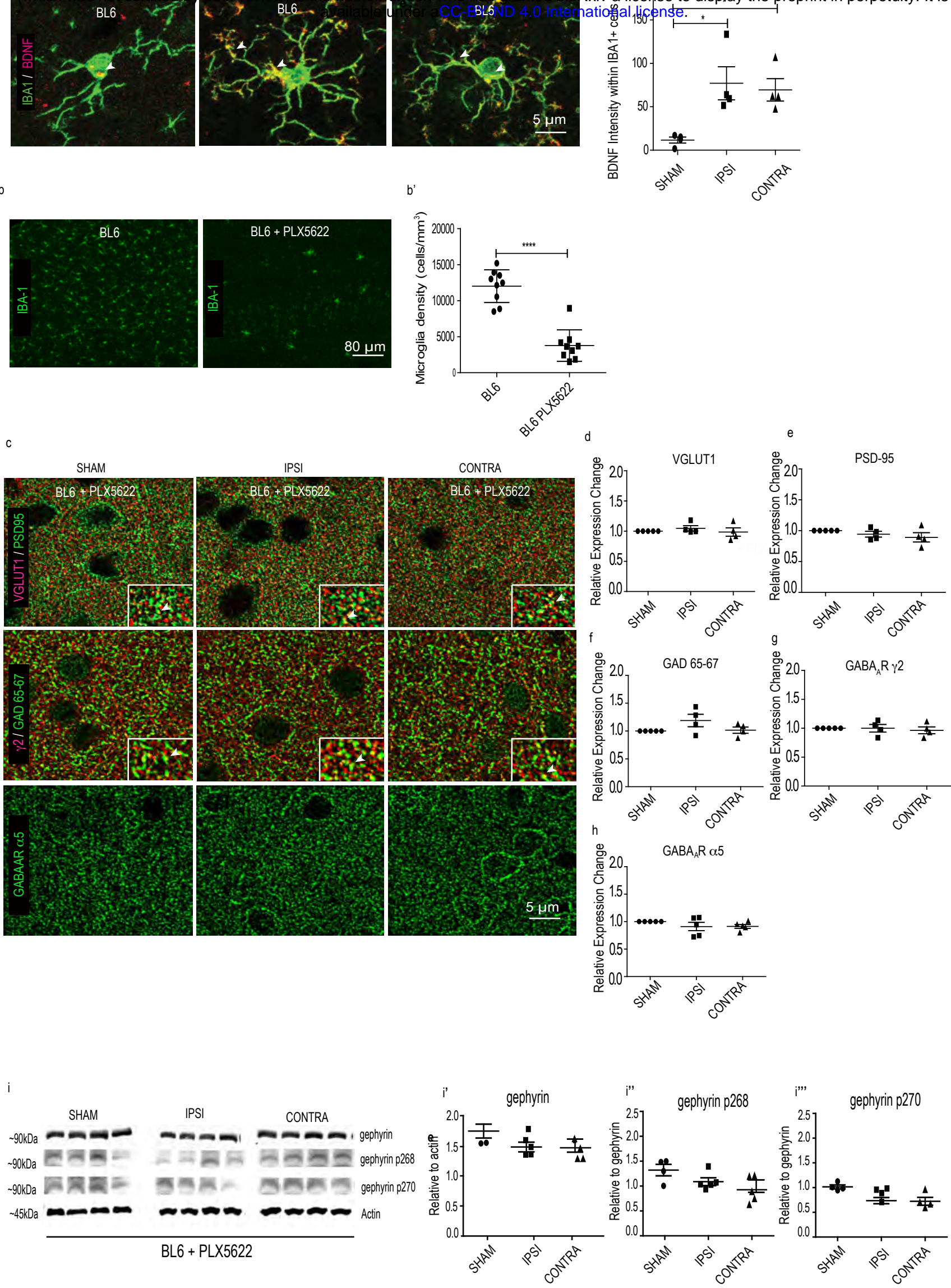

Cramer et al., Supplementary Figure 3 


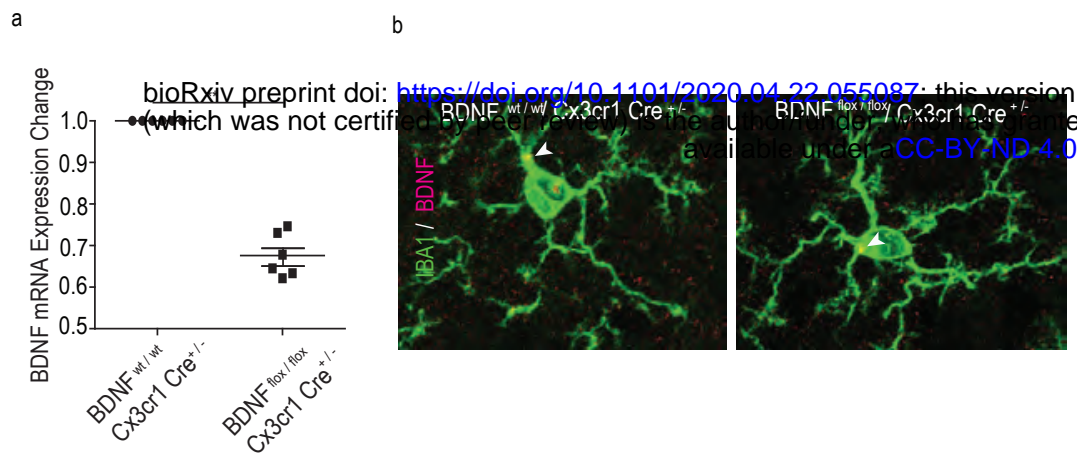

b' $\quad$ BDNF

BDNF 2020 . The copyright holder for this preprint ed SioR xiv a license to dlsplay the preprint in perpetuity. It is made Inferigationablicense.
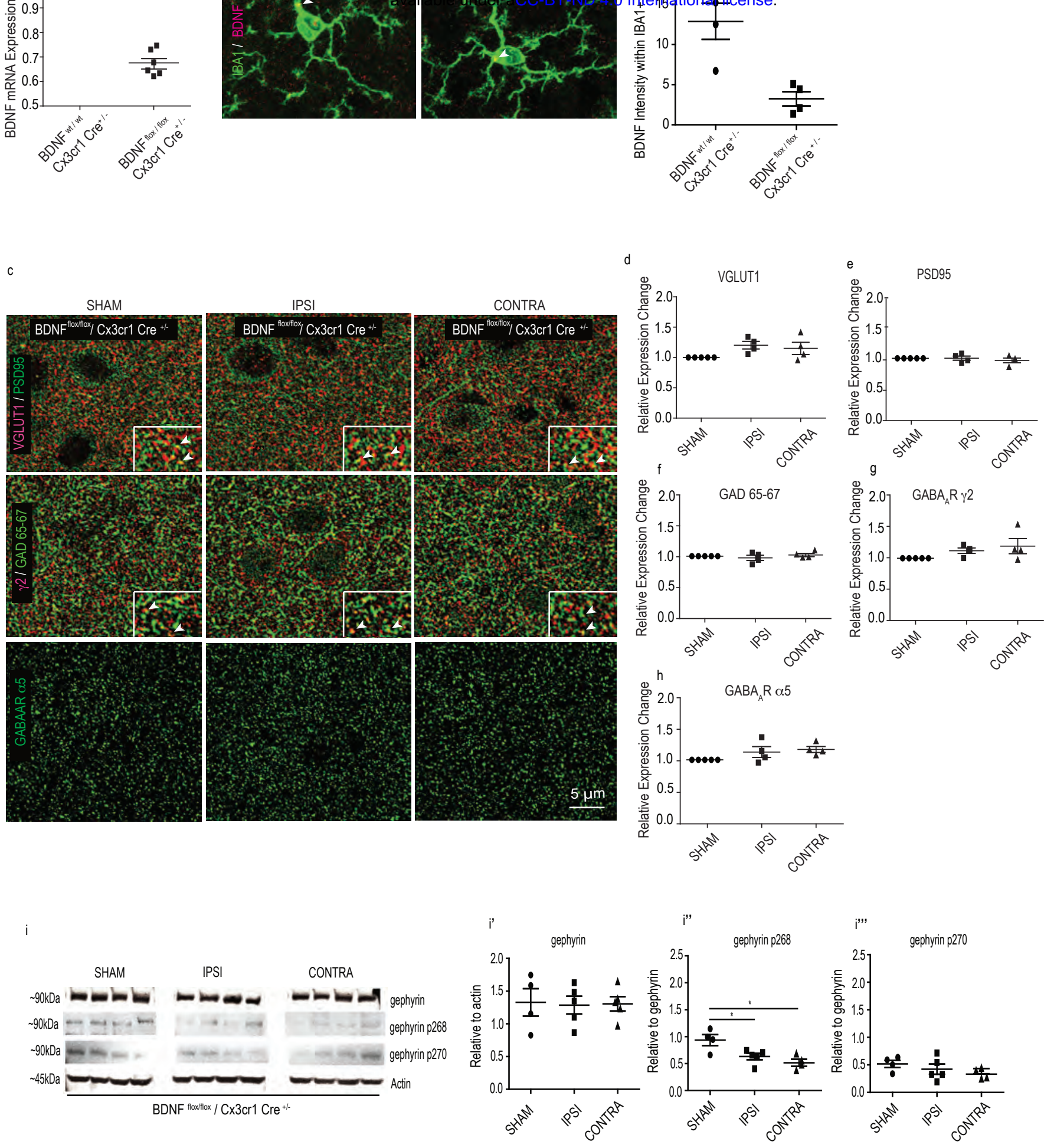

Cramer et al., Supplementary Figure 4 
bioRxiv prepriftRdoi: https://doi.org/10.PS 01/2020.04.22.055087; this version posted December 20, 2020. The copyright holder for this preprint

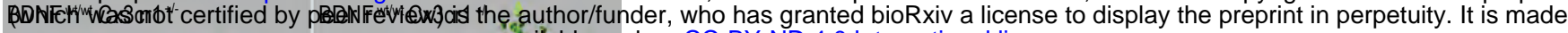
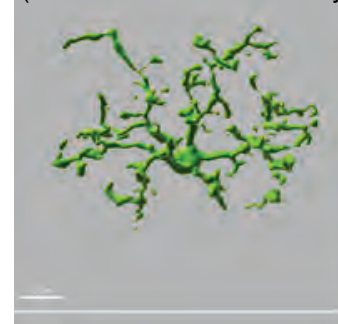

$\mathrm{BDNF}^{\mathrm{f} / \mathrm{fl}} \mathrm{C} \times 3 \mathrm{Cr}^{+1-}$

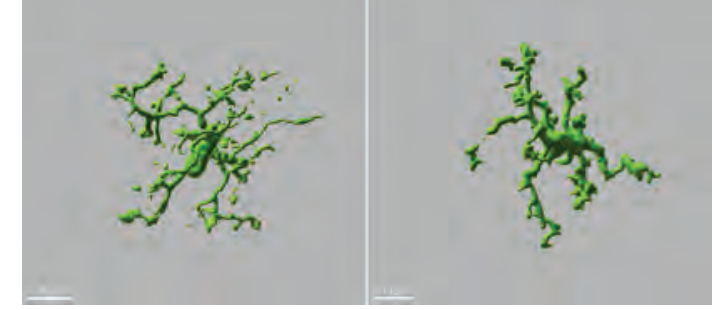
available under aCC-BY-ND 4.0 International license.

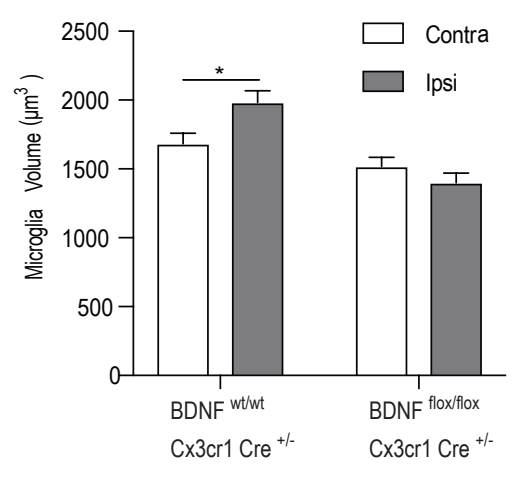

Cramer et al., Supplementary Figure 5 
a bioRxiv preprint doi: https://doi.org/10.1101/2020.04.22.055087; this version posted Decembefo30n2020. The copy"ight holder ffor this preprint (which was not certified by peer review) is the author/funder, who has granted bioRxiv a license to display the preprint in perpetuity. It is made

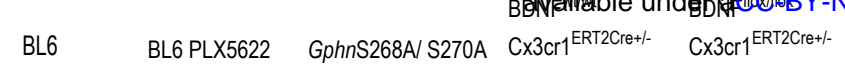

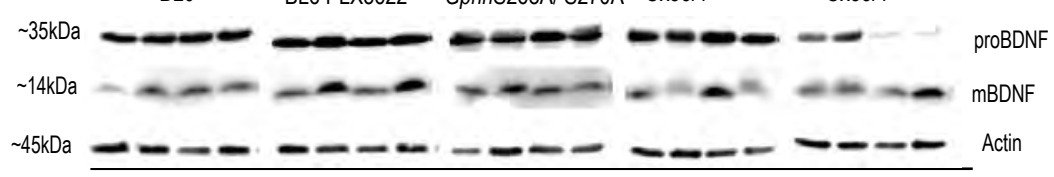

SHAM
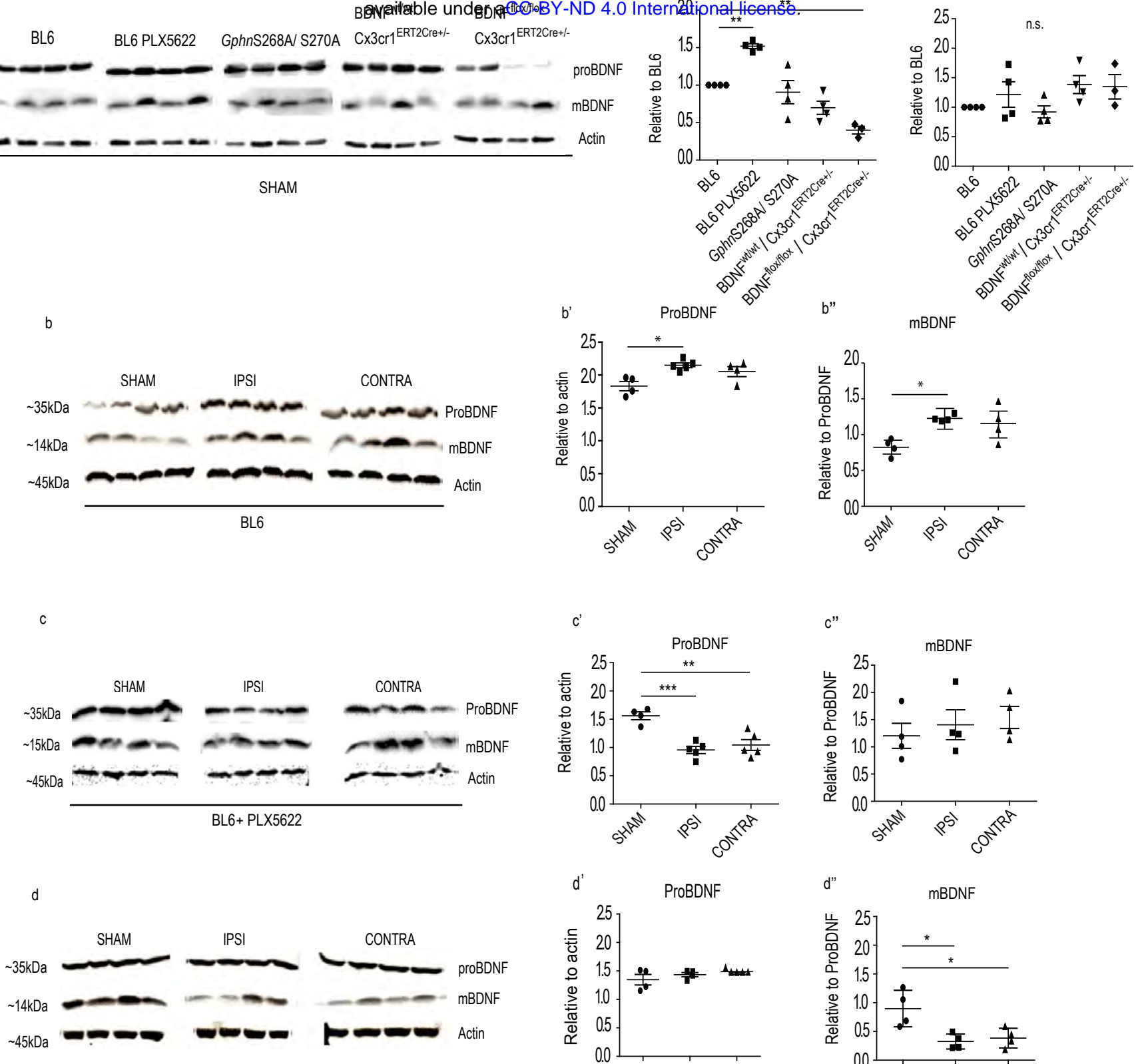

$\mathrm{BDNF}$ foxflox $/ \mathrm{C} \times 3 \mathrm{cr} 1 \mathrm{Cre}^{+/}$
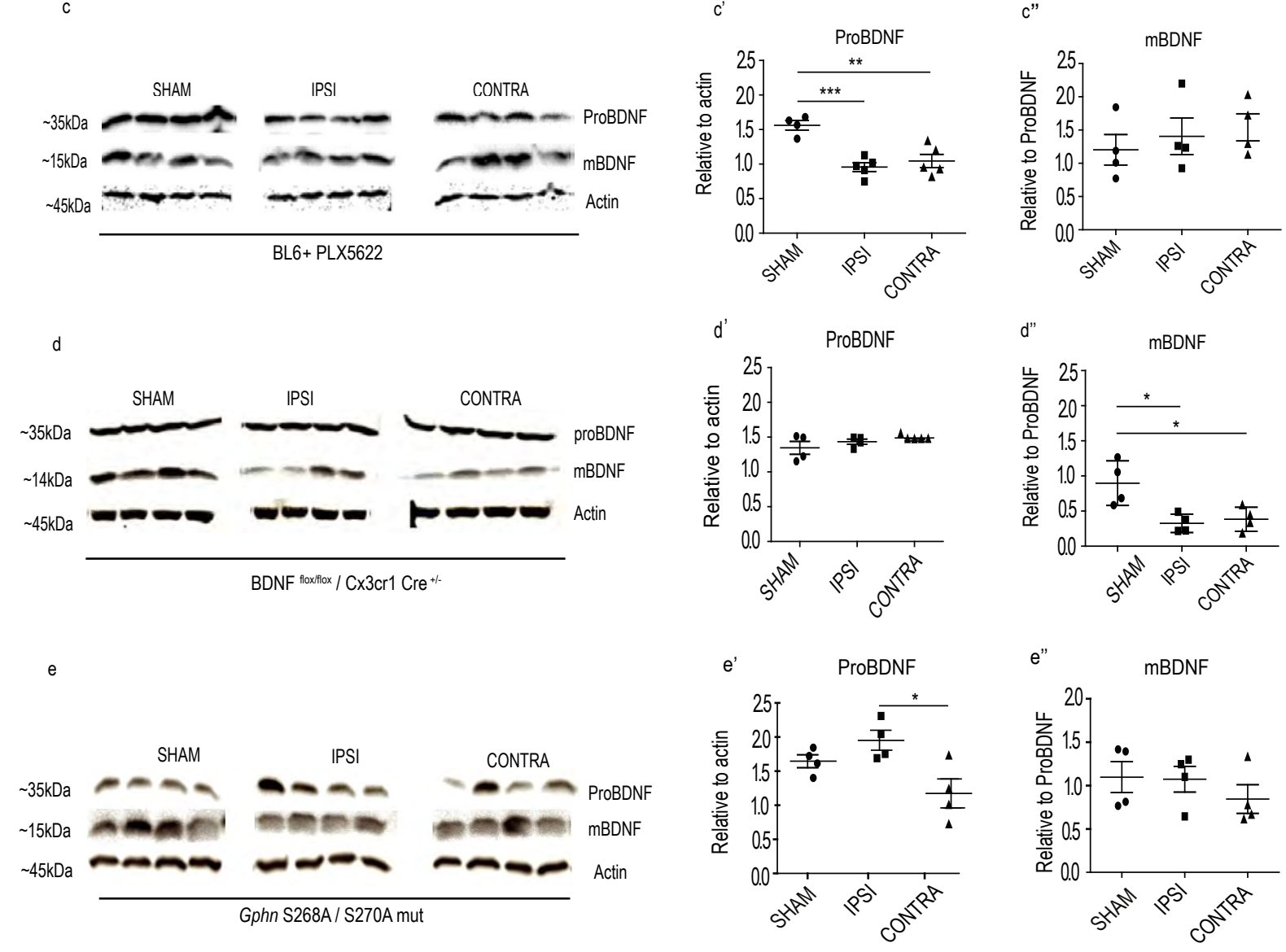

Cramer et al., Supplementary Figure 6 